\title{
Le théorème du symbole total d'un opérateur différentiel $p$-adique d'échelon $h \geq 0$
}

Zoghman Mebkhout

\begin{abstract}
Résumé
Dans cet article on démontre le théorème du symbole total d'un opérateur différentiel $p$-adique d'échelon $h \geq 0$ et la noethérianité de l'anneau des opérateurs différentiels $p$-adique d'échelon $h \geq 0$ audessus d'un ouvert †-adique affine lisse assez petit.

In this article we prove the total symbol theorem for the $p$-adic differential operators of degree $h \geq 0$ for the echelon filtration and the noetherianity of the ring of the $p$-adic differential operators of degree $h \geq 0$ for the echelon filtration over a $\dagger$-adic affine smooth scheme small enough.
\end{abstract}

\section{Introduction}

Cette article fait suite à l'article précédent [13] dont on utilise les définitions et les résultats. Les résultats de cet article ont été obtenus pour la plupart en collaboration avec L. Narvaez à la fin des années 1980 afin d'étudier les propriétés de finitude du faisceau des opérateurs différentiels $p$-adiques introduit dans les articles ([11], [12]) sur un schéma faiblement complet [15] qui est le faisceau naturel de base pour la théorie des coefficients de de Rham $p$-adiques et de la cohomologie de de Rham $p$-adique. Entre temps nous avons dû développer la difficile théorie des équations différentielles $p$-adiques sans laquelle on ne pourra pas atteindre des résultats significatifs dans cette théorie et qui est au fond du problème comme nous l'avons mis en évidence dans l'article fondamental de recherche [11].

2000 Mathematics Subject Classification : 14F30, $14 \mathrm{~F} 10$.

Keywords : p-adic differential operator, $p$-adic differential operator of $h \geq 0$ echelon, total symbol, division, continuity, noetherianity, $p$-adic de Rham cohomology. 
Les résultats de l'article précédent [13] et du présent article ne sont que des préliminaires sur les propriétés algébro-topologiques des anneaux des opérateurs différentiels $p$-adiques en dimensions supérieures qui ne fond pas appel à la théorie des équations différentielles $p$-adiques. Les outils essentiels pour l'article [13] et le présent article sont la théorie des espaces vectoriels topologiques localement convexes de type $\mathcal{L} \mathcal{F}$ sur un corps valué complet et les théorèmes de la division et de sa continuité dans les anneaux de séries entières éventuellement non commutatifs.

Nous étudions dans cet article la filtration par les échelons du faisceau des opérateurs différentiels $p$-adiques et ses propriétés de finitude. On utilise le théorème du symbole total les opérateurs différentiels $p$-adiques [13] pour démontrer le théorème du symbole total pour les opérateurs différentiels $p$-adiques d'échelon $h \geq 0$ et la noethérianité de l'anneau des sections globales du faisceau des opérateurs différentiels $p$-adiques d'échelon $h \geq 0$ audessus d'un ouvert affine assez petit.

Pour étudier les propriétés de finitude de l'algèbre des opérateurs différentiels sur un corps de caractéristique $p>0$ la méthode qui a fait ses preuves est d'introduire la $p$-filtration. C'est à l'aide la cette filtration que Chase [5] a montré que la dimension homologique plate de l'anneau des opérateurs différentiels d'une algèbre non singulière sur un corps parfait de caractéristique $p>0$ est égale à la dimension de l'algèbre et que Smith [19] a montré que la dimension homologique de l'anneau des opérateurs différentiels d'une algèbre non singulière sur un corps algébriquement clos de caractéristique $p>0$ est égale à la dimension de l'algèbre.

Nous avons défini, par analogie avec la $p$-filtration de l'anneau des opérateurs différentiels sur un corps de caractéristique $p>0$ ([5], [19]), la filtration par les échelons dans le contexte †-adique dans l'article de recherche [11] qui est adaptée à la cohomologie de de Rham $p$-adique. Un élément d'échelon $h \geq 0$ est toujours défini comme un opérateur différentiel et l'action des opérateurs différentiels sur le faisceau structural est essentielle dans notre point de vue. En inégales caractéristiques la $p$-filtration est l'image de la filtration par les échelons par réduction modulo l'idéal maximum. En caractéristique positive la filtration par les échelons coïncide avec la $p$-filtration.

P. Berthelot a défini les anneaux de niveau fini dans le contexte des schémas formels [2] qui ne sont pas en général des anneaux d'opérateurs différentiels. Pour une comparaison de ces points de vue nous renvoyons le lecteur à l'article de C. Noot-Huyghe [18].

Cependant le lecteur notera d'une part que les analogues dans le cas d'un schéma formel complet des résultats du présent article sont élémentaires et que d'autre part les puissances divisées n'interviennent pas dans notre point de vue. 
Nous remercions le Rapporteur de cet article pour ses remarques qui nous ont permis d'améliorer sa rédaction et pour sa relecture soigneuse.

Soient $V$ un anneau de valuation discrète complet d'inégales caractéristiques $(p, 0), A^{\dagger}$ une algèbre $\dagger$-adique lisse sur $V$ munie de coordonnées globales $x_{1}, \ldots, x_{n}$ et $D_{A^{\dagger} / V}^{\dagger, h}$ l'anneau des opérateurs différentiels $p$-adiques d'échelon $h \geq 0$ défini en 2.6.5.

Soient $h \geq 0$ un entier et un $n$-uplet d'entiers naturels $\beta^{j}, 0 \leq j \leq h$, notons $\left(\Delta_{x}^{p^{j}}\right)^{\beta^{j}}:=\left(\Delta_{1}^{p^{j}}\right)^{\beta_{1}^{j}} \ldots\left(\Delta_{n}^{p^{j}}\right)^{\beta_{n}^{h}}$ et $\mathbb{N}_{\leq p-1}^{n}$ l'ensemble des $n$-uples d'entiers naturels de composantes $\leq p-1$.

Dans cet article nous démontrons les deux théorèmes fondamentaux suivants :

Théorème 1.0.1 (3.1.2). Tout opérateur $P$ de $D_{A^{\dagger} / V}^{\dagger, h}$ s'écrit de manière unique :

$$
P\left(a, \Delta_{x}, \ldots, \Delta_{x}^{p^{h}}\right)=\sum_{\beta^{0}, \ldots, \beta^{h} \in\left(\mathbb{N}_{\leq p-1}^{n}\right)^{h} \times \mathbb{N}^{n}} a_{\beta^{0}, \ldots, \beta^{h}}\left(\Delta_{x}\right)^{\beta^{0}} \ldots\left(\Delta_{x}^{p^{h}}\right)^{\beta^{h}}
$$

où $a_{\beta^{0}, \ldots, \beta^{h}}$ est une suite d'éléments de l'algèbre $A^{\dagger}$ telle que la série symbole total

$$
\sigma_{P, h}(a, \xi):=P\left(a, \xi^{0}, \ldots, \xi^{h}\right):=\sum_{\beta^{0}, \ldots, \beta^{h} \in\left(\mathbb{N}_{\leq p-1}^{n}\right)^{h} \times \mathbb{N}^{n}} a_{\beta^{0}, \ldots, \beta^{h}}\left(\xi^{0}\right)^{\beta^{0}} \ldots\left(\xi^{h}\right)^{\beta^{h}}
$$

est un élément de l'algèbre commutative †-adique de type fini définie en 3.1.1. $\left(A^{\dagger}\left[\xi^{0}, \ldots, \xi^{h}\right]\right)^{\dagger}$.

De plus l'application symbole total $P(a, \Delta) \mapsto \sigma_{P, h}(a, \xi)$ est un isomorphisme de $A^{\dagger}$-modules à gauche entre l'anneau des opérateurs différentiels $D_{A^{\dagger} / V}^{\dagger, h}$ et l'algèbre $\left(A^{\dagger}\left[\xi^{0}, \ldots, \xi^{h}\right]\right)^{\dagger}$.

Bien entendu l'isomorphisme symbole total précédent ne respecte pas les structures multiplicatives et dépend des coordonnées locales.

Supposons de plus que l'algèbre $A^{\dagger}$ provient d'une $V$-algèbre de type fini lisse munie de coordonnées globales. On utilise alors le théorème précédent pour démontrer :

Théorème 1.0.2 (5.3.6). Sous les conditions précédentes l'anneau $D_{A^{\dagger} / V}^{\dagger, h}$ des opérateurs différentiels p-adiques d'échelon $h$ est noethérien.

Le premier théorème produit beaucoup d'exemples d'opérateurs différentiels $p$-adiques d'échelon $h \geq 0$. D'autre part la noethérianité est à la base des propriétés de finitude du faisceaux des opérateurs différentiels $p$-adique. Nous utilisons ces théorèmes de façon essentielle pour définir la cohomologie 
de de Rham $p$-adique d'une variété algébrique lisse admettant un relèvement comme schéma faiblement complet [15].

Voici le contenu de cet article. Le premier paragraphe rappelle la définition du faisceau des opérateurs différentiels sur un schéma faiblement complet. Dans le deuxième paragraphe nous définissons le faisceaux des opérateurs différentiels d'echelon $h \geq 0$ dans les différents contextes. Dans le troisième paragraphe nous montrons le théorème du symbole total pour les opérateurs différentiels $p$-adiques d'échelon $h \geq 0$ et la trivialité cohomologique du faisceau de tels opérateurs au-dessus d'un ouvert affine assez petit. Dans le quatrième paragraphe nous étudions quelques propriétés du faisceau des opérateurs différentiels $p$-adiques, l'idéal maximum de $V$ est contenu dans le radical et la transposition. Dans le cinquième paragraphe nous introduisons les anneaux de séries entières éventuellement non commutatifs $\left(V[Y, Z]_{\mathcal{R}}\right)^{\dagger}$ et nous montrons un théorème de division pour ces anneaux pour en déduire leur noethérianité et celle de l'anneau des opérateurs différentiels $p$-adiques d'échelon $h \geq 0$ au-dessus d'un ouvert affine assez petit. Les anneaux précédents généralisent et unifient de nombreuses situations, produisent beaucoup d'anneaux noethériens et la méthode de démonstration semble très générale. Dans le sixième paragraphe nous en déduisons la platitude de l'extension $\mathcal{D}_{\mathcal{X}^{\dagger} / V} \rightarrow \mathcal{D}_{\mathcal{X}^{\dagger} / V}^{\dagger}$ et la cohérence des faisceaux $\mathcal{D}_{\mathcal{X}^{\dagger} / V}^{\dagger, h}, \mathcal{D}_{\mathcal{X}^{\dagger} / K}^{\dagger, h}, \mathcal{D}_{\mathcal{X}^{\dagger} / K}^{\dagger}$ par la méthode classique de la théorie des faisceaux cohérents qui s'applique déjà pour montrer que le faisceau $\mathcal{D}_{X / k}$ des opérateurs différentiels $([22, \S 16])$ sur un schéma $X$ lisse sur un corps de caractéristique $p>0$ est cohérent cf. [5]. Dans le septième paragraphe nous montrons le théorème de comparaison entre la cohomologie définie par Monsky-Washnitzer [16] d'une variété affine non singulière sur un corps de caractéristique $p>0$ et la cohomologie de de Rham $p$-adique définie à l'aide de la théorie des $\mathcal{D}_{\mathcal{X}^{\dagger} / K^{-}}^{\dagger}$ modules ([11], [12]).

\section{Notations et conventions}

Dans cet article nous avons essayé d'être aussi soigneux que possible dans les notations et la terminologie. Afin d'éviter des répétitions nous utiliserons les notations suivantes :

1) $R$ anneau commutatif unitaire noethérien, $I$ idéal de $R, R_{s}:=R / I^{s}$, $s \geq 1$ en particulier $R_{1}:=R / I$,

2) $V, \mathfrak{m}, k, K, e$ anneau de valuation discrète complet d'inégales caractéristiques $(0, p)$ d'idéal maximal $\mathfrak{m}$, de corps résiduel $k$ et de corps de fractions $K$ et d'indice de ramification absolu $e:=v(p):=v_{\mathfrak{m}}(p)$, $|a|:=|a|_{p}:=p^{-v_{\mathfrak{m}}(p)}$ la valeur absolue $p$-adique d'un élément $a \in K$, 
3) $X / R$ schéma sur $R$,

4) De façon générale si $X / R$ est un espace annelé sur $R$ nous notons $\mathcal{D}_{X / R}$ le faisceau des opérateur différentiels défini par récurrence sur l'ordre dans $([22, \S 16])$ et noté $\mathcal{D}$ if $f_{R}\left(\mathcal{O}_{X / R}\right)$. La notation $\mathcal{D}_{X / R}$ et ses variantes sont aujourd'hui universelles,

4) Si $X / R$ est un schéma lisse sur $R([22, \S 17])$, un système de coordonnées locales $x_{1}, \ldots, x_{n}$ est constitué de sections locales du faisceau $\mathcal{O}_{X / R}$ telles que leurs différentielles forment une base locale du fibré des $R$-formes différentielles ([22, § 16]),

5) $\alpha \in \mathbb{N}^{n}, \alpha !:=\alpha_{1} ! \ldots \alpha_{n} !,|\alpha|:=\alpha_{1}+\cdots+\alpha_{n},\left(\begin{array}{c}\alpha \\ \beta\end{array}\right):=\alpha ! / \beta !(\alpha-\beta) !$, $x^{\alpha}:=x_{1}^{\alpha_{1}} \ldots x_{n}^{\alpha_{n}}$,

6) $\Delta_{x}^{\alpha}:=\Delta_{x_{1}}^{\alpha_{1}} \ldots \Delta_{x_{n}}^{\alpha_{n}}$ la suite des opérateurs différentiels associés à $x=$ $\left(x_{1}, \ldots, x_{n}\right)$ et à $\alpha=\left(\alpha_{1}, \ldots, \alpha_{n}\right) \in \mathbb{N}^{n}$ caractérisés par $\Delta_{x}^{\alpha}\left(x^{\beta}\right)=$ $\left(\begin{array}{l}\beta \\ \alpha\end{array}\right) x^{\beta-\alpha}([22, \S 16.11 .2])$,

7) Si $A$ est une $R$-algèbre on note $(A)^{\dagger}$ son complété faible pour la topologie $I$-adique de $R$ [16]. On dit qu'une $R$-algèbre $A^{\dagger}$ est une algèbre $†$-adique si elle coïncide avec son complété faible,

8) Si $A^{\dagger}$ est une $V$-algèbre $\dagger$-adique on note $A_{K}^{\dagger}$ l'algèbre $K \otimes_{V} A^{\dagger}$,

9) $\mathrm{Si}\left(V\left[Y_{1}, \ldots, Y_{m}\right]\right)^{\dagger} \rightarrow A^{\dagger} \rightarrow 0$ est une présentation, pour tout $\rho>1$ on note $A_{\rho}^{\dagger}$ l'image de la sous-algèbre des séries qui convergent dans le domaine $\left|Y_{i}\right| \leq \rho, i=1, \ldots, m$ et $A_{K, \rho}^{\dagger}:=K \otimes_{V} A_{\rho}^{\dagger}$. On note $\|-\|_{\rho}$ la norme quotient.

10) $\Omega_{A^{\dagger} / R}:=\Omega_{A^{\dagger} / R}^{s e p}$ le $A^{\dagger}$-module des formes différentielles séparées.

Pour simplifier nous utilisons les notations $|\alpha|:=\alpha_{1}+\cdots+\alpha_{n}$ au lieu de $|\alpha|_{\infty}$ et aussi $|a|:=p^{-v_{\mathfrak{m}}(a)}$ au lieu de $|a|_{p}$ en espérant que cela n'entraîne pas de confusion pour le lecteur.

Nous utilisons le critère de platitude locale communiqué par Grothendieck à Bourbaki, sous la forme suivante ([21, Chap. 0, 10.2.1] et [3, Chap. III, $\S 5$, no 2 , Thm. 1]).

Proposition 1.0.3. Soient deux $V$-algèbres noethériennes $D$ et $D^{\prime}$, telles que l'idéal $\mathfrak{m}$ est contenu dans le radical de $D^{\prime}$. Si $M$ est un $\left(D, D^{\prime}\right)$-bimodule qui est un $D^{\prime}$-module de type fini et que les réductions modulo $\mathfrak{m}^{s}$ de $M$ sont $D_{s}$-plates pour tout $s \geq 1$, alors $M$ est un $D$-module à gauche plat.

Par définition d'une $V$-algèbre, $V$ est contenu dans le centre de $D$. Les hypothèses de la proposition entraînent que $M$ est $D$-idéalement séparé pour la topologie m-adique, c'est-à-dire pour tout idéal $J$ à droite de $D$ le $D^{\prime}$ module à droite $J \otimes_{D} M$ est séparé pour la topologie m-adique. 


\section{Définition du faisceaux d'opérateurs différentiels $\dagger$-adiques d'échelon $h \geq 0$}

\subsection{Schémas †-adiques}

Soient $R$ un anneau unitaire commutatif noethérien, $I$ un idéal de $R$ et $\mathcal{X}^{\dagger}:=\left(X, \mathcal{O}_{\mathcal{X}^{\dagger} / R}\right)$ un $R$-schéma $\dagger$-adique [15]. On rappelle que $\mathcal{X}^{\dagger}:=$ $\left(X, \mathcal{O}_{\mathcal{X}^{\dagger} / R}\right)$ est un espace annelé tel que tout point admet un voisinage sur lequel l'espace annelé induit est isomorphe à un schéma †-adique affine. Un schéma †-adique affine se construit [15] à partir d'une algèbre $A^{\dagger}$ faiblement complète pour la topologie $I$-adique de $R$ et topologiquement de type fini [16], qui est noethérienne en vertu du théorème de Fulton, exactement comme se construit un schéma formel affine complet à partir d'un anneau noethérien adique complet [20]. Pour tout $s \geq 1$, la réduction modulo $I^{s}$ d'un schéma $†$-adique $\mathcal{X}^{\dagger}:=\left(X, \mathcal{O}_{\mathcal{X}^{\dagger} / R}\right)$ est un schéma $X_{s}:=\left(X, \mathcal{O}_{\mathcal{X}^{\dagger} / R} / I^{s}\right)$ localement de type fini sur $R_{s}$, en particulier l'espace topologique $X$ est localement noethérien et le faisceau structural $\mathcal{O}_{\mathcal{X}^{\dagger} / R}$, qui est un faisceau d'anneaux locaux par construction, est cohérent. Dans cet article nous dirons schéma †-adique pour schéma faiblement complet [15].

Définition 2.1.1. 1) On dit que le schéma †-adique $\mathcal{X}^{\dagger}$ est plat si le faisceau structural $\mathcal{O}_{\mathcal{X}^{\dagger} / R}$ est un faisceau de $R$-algèbres plates.

2) On dit que le schéma $\dagger$-adique $\mathcal{X}^{\dagger}$ est $\dagger$-adique lisse s'il est plat et que le schéma $\left(X, \mathcal{O}_{\mathcal{X}^{\dagger} / R} / I\right)$ est lisse sur $R_{1}$.

3) On dit qu'un ouvert $U$ de $X$ est un ouvert $\dagger$-affine si l'espace annelé induit $\mathcal{U}^{\dagger}:=\left(U, \mathcal{O}_{\mathcal{U}^{\dagger} / R}\right)$ est un schéma $\dagger$-adique affine.

4) On dit qu'un ouvert $U$ de $X$ à la propriété (diff), si le schéma †adique induit $\mathcal{U}^{\dagger}$ est $\dagger$-adique affine et lisse sur $R$ et que le faisceau des formes différentielles séparées est libre sur $\mathcal{O}_{\mathcal{U}^{\dagger} / R}$,

5) Si un ouvert $U$ a la propriété (diff), on dit que des éléments $\left(x_{1}, \ldots, x_{n}\right)$ de son algèbre $A^{\dagger}$ forment un système coordonnées locales audessus de $U$ si leurs différentielles $d x_{1}, \ldots, d x_{n}$ forme une $A^{\dagger}$-base $d u$ module des formes différentielles séparées $\Omega_{A^{\dagger} / R}$.

Attention : Il n'est pas du tout évident qu'un ouvert affine du schéma $\left(X, \mathcal{O}_{\mathcal{X}^{\dagger} / R} / I\right)$ au sens de la théorie des schémas est un ouvert †-affine ou même dans le contexte formel un ouvert formel-affine défini comme dans le cas $\nmid$-adique, mais les ouverts $\dagger$-affines forment une base de la topologie de $X$. 


\subsection{Le faisceau des opérateurs différentiels $†$-adiques $\mathcal{D}_{\mathcal{X}^{\dagger} / R}$ d'ordre localement fini}

On rappelle la définition du faisceau des opérateurs différentiels sur un espace annelé $([22, \S 16])$ :

Définition 2.2.1. On définit le faisceau des opérateurs différentiels $\mathcal{D}_{\mathcal{X}^{\dagger} / R}^{m}$ d'ordre $m \geq 0$ de l'espace annelé $\mathcal{X}^{\dagger}$ par récurrence sur $m$ comme le sousfaisceau du faisceau des $R$-endomorphismes $\mathcal{E}_{n d}\left(\mathcal{O}_{\mathcal{X}^{\dagger} / R}\right)$ du faisceau structural en posant $\mathcal{D}_{\mathcal{X}^{\dagger} / R}^{0}:=\mathcal{O}_{\mathcal{X}^{\dagger} / R}$ et en définissant le faisceau des opérateurs d'ordre $m \geq 1$ comme le sous-faisceau des endomorphismes dont le commutateur avec une section locale de $\mathcal{O}_{\mathcal{X}^{\dagger} / R}$ est un opérateur différentiel d'ordre $m-1$. Les faisceau des opérateurs différentiels d'ordre localement fini $\mathcal{D}_{\mathcal{X}^{\dagger} / R}$ est le faisceau filtré $\cup_{m \geq 0} \mathcal{D}_{\mathcal{X}^{\dagger} / R}^{m}$.

On rappelle le théorème suivant ([12, théorème A. 16, prop. 4.4.2]) qui est l'analogue †-adique du cas schématique ([22, Thm. 16.11.2]) mais dont la transposition n'est pas immédiate :

Théorème 2.2.2. Supposant que $\mathcal{X}^{\dagger}$ est $\dagger$-adique lisse sur $R$, alors pour tout $m \geq 0$ le faisceau $\mathcal{D}_{\mathcal{X}^{\dagger} / R}^{m}$ de $\mathcal{O}_{\mathcal{X}^{\dagger} / R^{-} \text {-modules est localement libre de }}$ type fini. Plus précisément soit un ouvert $U$ ayant la propriété (diff) et $\left(x_{1}, \ldots, x_{n}\right)$ un système de coordonnées locales au-dessus de $U$, alors il existe une unique famille d'opérateurs différentiels $\Delta^{\alpha}:=\Delta_{x}^{\alpha}, \alpha \in \mathbb{N}^{n}$ caractérisée par les relations $\Delta^{\alpha}\left(x^{\beta}\right)=\left(\begin{array}{c}\beta \\ \alpha\end{array}\right) x^{\beta-\alpha}$ et tels que les opérateurs différentiels $\Delta^{\alpha},|\alpha| \leq m$ forment une base locale $d u \mathcal{O}_{\mathcal{X}^{\dagger} / R^{-m o d u l e}} \mathcal{D}_{\mathcal{X}^{\dagger} / R}^{m}$.

Par construction les ouverts affines ayant la propriété (diff ) forment une base de la topologie de $X$.

\subsection{Le faisceau des opérateurs différentiels †-adiques $\mathcal{D}_{\mathcal{X}^{\dagger} / R}^{\dagger} \mathrm{d}$ 'ordre infini}

Rappelons la définition du faisceau le faisceau opérateurs différentiels †-adiques d'ordre infini introduit dans les articles ([11], [12]) :

Définition 2.3.1. Soit $\mathcal{X}^{\dagger}:=\left(X, \mathcal{O}_{\mathcal{X}^{\dagger} / R}\right)$ un schéma $\dagger$-adique (non nécessairement lisse), on définit le faisceau des opérateurs différentiels $\mathcal{D}_{\mathcal{X}^{\dagger} / R}^{\dagger}$ d'ordre infini de l'espace annelé $\mathcal{X}^{\dagger}$ comme le sous-faisceau du faisceau des $R$ endomorphismes $\mathcal{E}_{n d_{R}}\left(\mathcal{O}_{\mathcal{X}^{\dagger} / R}\right)$ du faisceau structural dont la réduction modulo l'idéal $I^{s}$ pour tout $s \geq 1$ est un opérateur différentiel sur le $R_{s}$-schéma $X_{s}:=\left(X, \mathcal{O}_{\mathcal{X}^{\dagger} / R} / I^{s}\right)$ et dont l'ordre est localement borné par une fonction linéaire en $s$. 
Le faisceau $\mathcal{D}_{\mathcal{X}^{\dagger} / R}^{\dagger}$ est donc par construction un sous-faisceau de $\mathcal{O}_{\mathcal{X}^{\dagger} / R^{-}}$ algèbres du faisceau des endomorphismes $\mathcal{E} n d_{R}\left(\mathcal{O}_{\mathcal{X}^{\dagger} / R}\right)$ qui contient comme

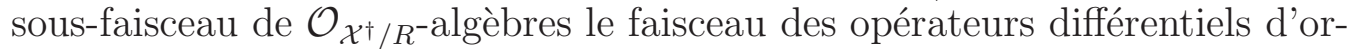
dre localement fini $\mathcal{D}_{\mathcal{X}^{\dagger} / R}$.

Remarque 2.3.2. Si on part d'un schéma formel complet $\mathcal{X}:=\left(X, \mathcal{O}_{\mathcal{X} / R}\right)$, [20], (non nécessairement lisse) les deux définitions précédentes se transpose sans changement et l'on obtient le couple $\mathcal{D}_{\mathcal{X} / R} \subset \mathcal{D}_{\mathcal{X} / R}^{\dagger}$ dont l'étude est élémentaire dans le cas d'un schéma formel formellement lisse mais qui n'a pas prise sur les questions de fond.

2.4. Définition du faisceau des opérateurs différentiels de degré $h \geq 0$ pour la $p$-filtration, cas d'un schéma sur un corps de caractéristique $p>0$

Soit un schéma $X$ sur un corps $k$ de caractéristique $p>0$. Par définition $([22, \S 16])$ le faisceau des opérateurs différentiels $\mathcal{D}_{X / k}$ est un sous-faisceau du faisceau $\mathcal{E} n d_{k}\left(\mathcal{O}_{X / k}\right)$ des endomorphismes du faisceau structural. Pour tout entier $h \geq 0$ on note $\mathcal{O}_{X / k}, h+1$ le sous-faisceau de $k$-algèbres engendré par l'image dans le faisceau $\mathcal{O}_{X / k}$ de l'élévation à la puissance $p^{h+1}$. On a la définition ([5], [19]) :

Définition 2.4.1. Pour tout entier $h \geq 0$ on appelle faisceau des opérateurs de degré $h$ pour la p-filtration le sous-faisceau $\mathcal{D}_{X / k}^{<\infty, h}:=\mathcal{E} n d_{\mathcal{O}_{X / k}, h+1}\left(\mathcal{O}_{X / k}\right)$ du faisceau des endomorphismes du faisceau structural qui sont $\mathcal{O}_{X / k, h+1^{-}}$ linéaires.

Cette définition est justifiée par le résultat suivant :

Théorème 2.4.2. Si le schéma $X$ est localement de type fini sur $k$, pour tout entier $h \geq 0$, tout opérateur de degré $h$ pour la $p$-filtration est un opérateur différentiel et la filtration croissante $\mathcal{D}_{X / k}^{<\infty, h}$ indexée par les entiers $h \geq 0$ est une filtration exhaustive du faisceau $\mathcal{D}_{X / k}$ par des sous-faisceaux de $\mathcal{O}_{X / k}$-algèbres.

Démonstration. La question est locale, on peut supposer que $X$ est affine d'algèbre $A$. Soit $J$ l'idéal de l'algèbre $A \otimes_{k} A$ noyau de la multiplication $A \otimes_{k} A \rightarrow A$. Alors le module $\operatorname{End}_{k}(A)$ est une $A \otimes_{k} A$-algèbre et $D_{A / k}$ est une sous- $A \otimes_{k} A$-algèbre. Un endomorphisme de $\operatorname{End}_{k}(A)$ est un opérateur différentiel d'ordre $m$ si et seulement s'il est annulé par $J^{m+1}([22, \S 16])$. Soient $y_{1}, \ldots, y_{m}$ un système de générateurs de $A$ et $P$ un opérateur de degré $h \geq 0$ pour la $p$-filtration, alors $P$ est un opérateur différentiel d'ordre borné par $m p^{h+1}-1$. En effet $J^{m p^{h+1}}$ est engendré par $y_{i}^{p^{h+1}} \otimes 1-1 \otimes y_{i}^{p^{h+1}}$, 
$i=1, \ldots, m$ et $\left(y_{i}^{p^{h+1}} \otimes 1-1 \otimes y_{i}^{p^{h+1}}\right) P=0$. Réciproquement il est facile de voir qu'un opérateur différentiel commute à $y_{i}^{p^{h+1}}$ pour $h$ assez grand et donc commute à l'action de toutes les puissances d'ordre $p^{h+1}$.

Corollaire 2.4.3. Supposons que le schéma $X$ est lisse sur $k$, alors pour tout $h \geq 0$ le faisceau $\mathcal{D}_{X / k}^{<\infty, h}$ de $\mathcal{O}_{X / k}$-algèbres est localement engendré par les opérateurs différentiels d'ordre $\leq p^{h}$.

Démonstration. Soit $x=\left(x_{1}, \ldots, x_{n}\right)$ un système de coordonnées locales, alors on a de façon évidente l'inclusion $\mathcal{O}_{X / k}\left[\Delta ., \ldots, \Delta^{p^{h}}\right] \subset \mathcal{D}_{X / k}^{<\infty, h}$. Comme $\left(\Delta_{i}^{p^{h}}\right)^{p}=0$ il facile de voir que tout opérateur d'échelon $h$ est d'ordre $<p^{h+1}$ en $\Delta_{i}$ pour tout $i, 1 \leq i \leq n$. Si $\alpha=\left(\alpha_{1}, \ldots, \alpha_{n}\right), \alpha_{i}<p^{h+1}$ on peut écrire $\alpha_{i}=a_{i, 0}+\cdots+a_{i, h} p^{h}, 0 \leq a_{i, j} \leq p-1, \alpha=\beta^{0}+\cdots+\beta^{h} p^{h}$ et

$$
\Delta^{\alpha}=u_{h}(\Delta)^{\beta^{0}} \ldots\left(\Delta^{p^{h}}\right)^{\beta^{h}}
$$

où $u_{h}$ est un nombre rationnel qui est unité $p$-adique. Ceci montre l'inclusion $\mathcal{O}_{X}\left[\Delta, \ldots, \Delta^{p^{h}}\right] \supset \mathcal{D}_{X / k}^{<\infty, h}$

Notation 2.4.4. Pour deux entiers $m \geq 0, h \geq 0$ nous notons $\mathcal{D}_{X / k}^{m, h}$ le $\mathcal{O}_{X / k^{-}}$ module des opérateurs différentiels d'ordre borné par $m$ et d'échelon borné par $h$. L'indice de gauche donne l'information sur l'ordre et celui de droite sur l'échelon.

\subsection{Définition du faisceau des opérateurs différentiels d'echelon $h \geq 0$, cas d'un $\mathbb{Z}_{(p)}$-schéma}

Le corollaire 2.4.3 permet d'étendre la définition de la $p$-filtration au cas d'un schéma où tous les entiers premiers à $p$ sont inversibles. Soit $X$ un schéma au-dessus de l'anneau $\mathbb{Z}_{(p)}$ localisé de $\mathbb{Z}$ en l'idéal $p \mathbb{Z}$.

Définition 2.5.1. Soit $h \geq 0$ un entier, on définit le faisceau $\mathcal{D}_{X / \mathbb{Z}_{(p)}^{<\infty}}^{<\text {des }}$ opérateurs différentiels d'échelon $h$ comme le faisceau de sous $\mathcal{O}_{X / \mathbb{Z}_{(p)}}$ algèbres de la $\mathcal{O}_{X / \mathbb{Z}_{(p)}}$-algèbre $\mathcal{D}_{X / \mathbb{Z}_{(p)}}$ localement engendré par les opérateurs d'ordre $\leq p^{h}$.

En vertu du corollaire 2.4.3 la filtration par les échelons coïncide avec la $p$-filtration dans le cas où l'anneau de base est un corps de caractéristique $p>0$.

Proposition 2.5.2. Soit $X$ un schéma lisse sur $\mathbb{Z}_{(p)}$, alors la filtration par les échelons $\mathcal{D}_{X / \mathbb{Z}_{(p)}}^{<\infty, h}$ est une filtration croissante exhaustive du faisceau de opérateurs différentiels $\mathcal{D}_{X / \mathbb{Z}_{(p)}}$ par des sous-faisceaux de $\mathcal{O}_{X / \mathbb{Z}_{(p)}}$-algèbres. 
Démonstration. En effet si $x=\left(x_{1}, \ldots, x_{n}\right)$ un système de coordonnées locales, pour tout entier $h \geq 0$ on a par définition l'inclusion

$$
\mathcal{O}_{X / \mathbb{Z}_{(p)}}\left[\Delta ., \ldots, \Delta \cdot^{p^{h}}\right] \subset \mathcal{D}_{X / \mathbb{Z}_{(p)}^{<\infty, h}}^{<\infty}
$$

qui est une égalité de façon évidente.

Si $m$ est un entier et $m=a_{h} p^{h_{m}}+\cdots+a_{0}$ son développement $p$-adique alors tout opérateur différentiel d'ordre borné par $m$ est nécessairement d'échelon borné par $h_{m}$, d'où l'inclusion

$$
\mathcal{D}_{X / \mathbb{Z}_{(p)}}^{m} \subset \mathcal{D}_{X / \mathbb{Z}_{(p)}}^{<\infty, h_{m}},
$$

qui montre que la filtration par les échelons est exhaustive.

\subsection{Définition du faisceau des opérateurs différentiels d'echelon $h \geq 0$, cas d'un schéma †-adique}

Soient $R$ un anneau commutatif unitaire noethérien qui est un $\mathbb{Z}_{(p)}$ algèbre, $I$ un idéal de $R$ et $\mathcal{X}^{\dagger}:=\left(X, \mathcal{O}_{\mathcal{X}^{\dagger} / R}\right)$ un $R$-schéma $\dagger$-adique pour la topologie $I$-adique de $R$.

Définition 2.6.1. Soit $h \geq 0$ un entier, on définit le faisceau $\mathcal{D}_{\mathcal{X}^{\dagger} / R}^{<\infty}$ des opérateurs différentiels d'ordre localement fini et d'échelon $h$ comme le faisceau de sous $\mathcal{O}_{\mathcal{X}^{\dagger} / R^{-a l g e ̀ b r e s ~ d u ~ f a i s c e a u ~}} \mathcal{D}_{\mathcal{X}^{\dagger} / R}$ localement engendré par les opérateurs d'ordre $\leq p^{h}$.

Notation 2.6.2. Pour un multi-indice $\beta^{j}=\left(\beta_{1}^{j}, \ldots, \beta_{n}^{j}\right)$ on note $\left(\Delta^{p^{j}}\right)^{\beta^{j}}$ l'opérateur différentiel $\left(\Delta_{1}^{p^{j}}\right)^{\beta_{1}^{j}} \ldots\left(\Delta_{n}^{p^{j}}\right)^{\beta_{n}^{j}}$ et pour $\beta=\left(\beta^{0}, \ldots, \beta^{h}\right)$ on note l'opérateur différentiel $(\Delta)^{\beta}:=\left(\Delta^{p^{0}}\right)^{\beta^{0}} \ldots\left(\Delta^{p^{n}}\right)^{\beta^{n}}$.

Proposition 2.6.3. Si le schéma $\dagger$-adique $\left(X, \mathcal{O}_{\mathcal{X}^{\dagger} / R}\right)$ est $\dagger$-adique lisse alors la filtration par les échelons $\mathcal{D}_{\mathcal{X}^{\dagger} / R}^{<\infty, h}$ est une filtration croissante exhaustive du faisceau de opérateurs différentiels d'ordre fini $\mathcal{D}_{\mathcal{X}^{\dagger} / R}$ par des

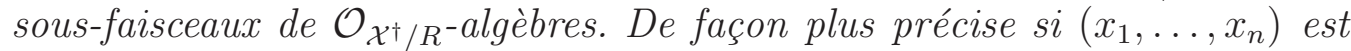
un système de coordonnées locales au-dessus d'un ouvert ayant la propriété (diff) d'algèbre $A^{\dagger}$, pour tout $h \geq 0$, tout opérateur différentiel $P$ d'échelon $h$ s'écrit de manière unique en une somme finie

$$
P\left(a, \Delta, \ldots, \Delta^{p^{h}}\right)=\sum_{\beta^{0}, \ldots, \beta^{h} \in\left(\mathbb{N}_{\leq p-1}^{n}\right)^{h} \times \mathbb{N}^{n}} a_{\beta^{0}, \ldots, \beta^{h}}(\Delta)^{\beta^{0}} \ldots\left(\Delta^{p^{h}}\right)^{\beta^{h}}
$$

où $a_{\beta^{0}, \ldots, \beta^{h}}$ est un élément de l'algèbre $A^{\dagger}$. 
Démonstration. C'est une conséquence du théorème 2.2.2 montrant que les opérateurs $\Delta^{\alpha}$ forment une base locale du faisceau de $\mathcal{O}_{\mathcal{X}^{\dagger} / R^{-m o d u l e s}} \mathcal{D}_{\mathcal{X}^{\dagger} / R}$.

Pour tout $h \geq 0$ nous pouvons considérer le complété $I$-adique $\mathcal{D}_{\mathcal{X} / R}^{\wedge, h}$ du faisceau $\mathcal{D}_{\mathcal{X}^{\dagger} / R}^{<\infty, h}$. Nous pouvons aussi considérer le complété $I$-adique $\left(X, \mathcal{O}_{\mathcal{X} / R}\right)$ du schéma $\left(X, \mathcal{O}_{\mathcal{X}^{\dagger} / R}\right)$ et le faisceau $\mathcal{D}_{\mathcal{X} / R}^{\wedge, h}$ opère à gauche de façon naturelle sur le faisceau $\mathcal{O}_{\mathcal{X} / R}$ qui apparaît comme un sous-faisceau de $R$-algèbres du faisceau des endomorphismes $\mathcal{E} n d_{R}\left(\mathcal{O}_{\mathcal{X} / R}\right)$.

Pour tout entier $s \geq 1$ nous pouvons considérer la réduction modulo $I^{s}$

$$
\mathcal{D}_{\mathcal{X}^{\dagger} / R}^{<\infty, h} / I^{s}=\mathcal{D}_{\mathcal{X} / R}^{\wedge, h} / I^{s}
$$

du faisceau $\mathcal{D}_{\mathcal{X} / R}^{\wedge, h}$. C'est de façon naturelle un faisceau de $\mathcal{O}_{X_{s}}$-algèbres filtré sur le schéma $X_{s}$ réduction modulo l'idéal $I^{s}$ du schéma †-adique $\left(X, \mathcal{O}_{\mathcal{X}^{\dagger} / R}\right)$.

Le faisceau $\mathcal{D}_{\mathcal{X}^{\dagger} / R}^{\dagger}$ opère à gauche de façon naturelle sur le faisceau $\mathcal{O}_{\mathcal{X} / R}$ qui apparaît aussi comme un sous-faisceau de $R$-algèbres du faisceau des endomorphismes $\mathcal{E} n d_{R}\left(\mathcal{O}_{\mathcal{X} / R}\right)$. On peut donc pour tout $h \geq 0$ considérer l'intersection $\mathcal{D}_{\mathcal{X}^{\dagger} / R}^{\dagger} \cap \mathcal{D}_{\mathcal{X} / R}^{\wedge, h}$ prise dans le faisceau $\mathcal{E}_{n d_{R}}\left(\mathcal{O}_{\mathcal{X} / R}\right)$. L'inclusion $\mathcal{D}_{\mathcal{X}^{\dagger} / R}^{<\infty, h} \subset \mathcal{D}_{\mathcal{X}^{\dagger} / R}$ induit un morphisme filtré

$$
\mathcal{D}_{\mathcal{X}^{\dagger} / R}^{<\infty, h} / I^{s} \rightarrow \mathcal{D}_{X_{s} / R_{s}}
$$

pour tout $s \geq 1$ qui n'est pas injectif bien sûr mais dont l'image est le faisceau $\mathcal{D}_{X_{s} / R_{s}}^{<\infty, h}$. Cependant :

Proposition 2.6.4. Si l'idéal I est engendré par un élément $b$ non diviseur de zéro dans $R$ et si le schéma $\dagger$-adique $\mathcal{X}^{\dagger}:=\left(X, \mathcal{O}_{\mathcal{X}^{\dagger} / R}\right)$ est $\dagger$-adique lisse, l'inclusion $\mathcal{D}_{\mathcal{X}^{\dagger} / R}^{\dagger} \cap \mathcal{D}_{\mathcal{X} / R}^{\wedge, h} \subset \mathcal{D}_{\mathcal{X} / R}^{\wedge, h}$ induit un isomorphisme

$$
\left(\mathcal{D}_{\mathcal{X}^{\dagger} / R}^{\dagger} \cap \mathcal{D}_{\mathcal{X} / R}^{\wedge, h}\right) / I^{s} \simeq \mathcal{D}_{\mathcal{X} / R}^{\wedge, h} / I^{s}
$$

pour tout $s \geq 1$.

Démonstration. Seule l'injectivité pose problème. Il faut montrer que l'inclusion

$$
I^{s}\left(\mathcal{D}_{\mathcal{X}^{\dagger} / R}^{\dagger} \cap \mathcal{D}_{\mathcal{X} / R}^{\wedge, h}\right) \subset \mathcal{D}_{\mathcal{X}^{\dagger} / R}^{\dagger} \cap I^{s} \mathcal{D}_{\mathcal{X} / R}^{\wedge, h}
$$

est une égalité. La question est locale. Si $x=\left(x_{1}, \ldots, x_{n}\right)$ sont des coordonnées locales au dessus d'un ouvert †-adique $U$ ayant la propriété (diff) d'algèbre $A^{\dagger}$ et si un opérateur $P \operatorname{de} \Gamma\left(U, \mathcal{D}_{\mathcal{X}^{\dagger} / R}^{\dagger}\right)$ appartient à $I^{s} \Gamma\left(U, \mathcal{D}_{\mathcal{X} / R}^{\wedge, h}\right)$ alors nécessairement les coefficients

$$
a_{\alpha}=\sum_{0 \leq \beta \leq \alpha}\left(\begin{array}{l}
\alpha \\
\beta
\end{array}\right)(-x)^{\beta} P\left(x^{\alpha-\beta}\right)
$$


appartiennent à $I^{s} A^{\wedge}$, donc à

$$
I^{s} A^{\dagger}=A^{\dagger} \cap I^{s} A^{\wedge} \quad \text { et } P \text { appartient à } I^{s} \Gamma\left(U, \mathcal{D}_{\mathcal{X}^{\dagger} / R}^{\dagger}\right) \text {. }
$$

En fait les coefficients $a_{\alpha}$ appartiennent à l'idéal engendré par $b^{s}$. L'égalité

$$
b^{s} P^{\prime}=b^{s} P^{h}
$$

pour un opérateur $P^{\prime}$ de $\Gamma\left(U, \mathcal{D}_{\mathcal{X}^{\dagger} / R}^{\dagger}\right)$ et un opérateur $P^{h}$ de $\Gamma\left(U, \mathcal{D}_{\mathcal{X}^{\wedge} / R}^{\wedge, h}\right)$ entraîne que l'opérateur $P^{h}$ opère sur $A^{\dagger}$ puisque l'action de $b$ sur l'anneau $A^{\wedge}$ est injective par platitude. L'opérateur $P^{h}$ est un élément de $\Gamma\left(U, \mathcal{D}_{\mathcal{X}^{\dagger} / R}^{\dagger}\right)$, d'où l'égalité de l'inclusion précédente.

Pour tout entier $s \geq 1$ le faisceau

$$
\mathcal{D}_{\mathcal{X}^{\dagger} / R}^{<\infty h} / I^{s}=\mathcal{D}_{\mathcal{X} / R}^{\wedge, h} / I^{s}
$$

est filtré par la filtration quotient de la filtration par l'ordre des opérateurs différentiels du faisceau $\mathcal{D}_{\mathcal{X}^{\dagger} / R}^{<\infty, h}$. Nous arrivons à la définition fondamentale :

Définition 2.6.5. Soit un entier $h \geq 0$ et $\mathcal{X}^{\dagger}=\left(X, \mathcal{O}_{\mathcal{X}^{\dagger} / R}\right)$ un $R$-schéma $\dagger$ adique lisse, on définit le faisceau des opérateurs d'ordre infini et d'échelon $h$ noté $\mathcal{D}_{\mathcal{X}^{\dagger} / R}^{\dagger, h}$, comme le sous-faisceau du faisceau $\mathcal{D}_{\mathcal{X}^{\dagger} / R}^{\dagger} \cap \mathcal{D}_{\mathcal{X} / R}^{\wedge, h}$ des opérateurs dont l'image par le morphisme

$$
\left(\mathcal{D}_{\mathcal{X}^{\dagger} / R}^{\dagger} \cap \mathcal{D}_{\mathcal{X} / R}^{\wedge, h}\right) / I^{s} \rightarrow \mathcal{D}_{\mathcal{X} / V}^{\wedge, h} / I^{s}
$$

est d'ordre localement borné par une fonction linéaire en $s$.

Remarque 2.6.6. Le lecteur prendra garde que l'inclusion

$$
\mathcal{D}_{\mathcal{X}^{\dagger} / R}^{\dagger, h} \subset \mathcal{D}_{\mathcal{X}^{\dagger} / R}^{\dagger} \cap \mathcal{D}_{\mathcal{X} / R}^{\wedge, h}
$$

est stricte. En fait par construction le faisceau $\mathcal{D}_{\mathcal{X}^{\dagger} / R}^{\dagger, h}$ est un sous-faisceau du faisceau $\mathcal{D}_{\mathcal{X} / R}^{\wedge, h}$. Un opérateur $P$ de ce dernier faisceau, c'est-à-dire une section de ce faisceau, est une section du premier faisceau si :

1) $P$ opère à gauche sur le faisceau $\mathcal{O}_{\mathcal{X}^{\dagger} / R}$ comme endomorphisme de faisceau $R$-linéaire,

2) il a condition du degré par réduction modulo les puissances de $I$. Cela entraîne que c'est un $R$-endomorphisme du faisceau structural $\mathcal{O}_{\mathcal{X}^{\dagger} / R}$ dont la réduction modulo les puissances de $I$ est un opérateur différentiel qui a la propriété du degré. C'est en particulier un opérateur de $\mathcal{D}_{\mathcal{X}^{\dagger} / R}^{\dagger}$. 
3) On a pour tout $h \geq 0$ le diagramme commutatif d'inclusions de faisceaux sur $X$ muni de la topologie de Zariski :

$$
\begin{array}{cccccc}
\mathcal{D}_{\mathcal{X}^{\dagger} / R}^{\dagger, h} & \subset & \mathcal{D}_{\mathcal{X}^{\dagger} / R}^{\dagger} & \subset & \mathcal{E}_{n d_{R}}\left(\mathcal{O}_{\mathcal{X}^{\dagger} / R}\right) \\
\cap & & \cap & & \cap \\
\mathcal{D}_{\mathcal{X} / R}^{\wedge, h} & \subset & \mathcal{D}_{\mathcal{X} / R}^{\dagger} & \subset & \mathcal{E}_{n} d_{R}\left(\mathcal{O}_{\mathcal{X} / R}\right) .
\end{array}
$$

Proposition 2.6.7. Pour tout $h \geq 0$ le faisceau $\mathcal{D}_{\mathcal{X}^{\dagger} / R}^{\dagger, h}$ est un sous-faisceau d'anneaux des cinq autres faisceaux du diagramme précédent.

Démonstration. En effet si $P_{1}, P_{2}$ opèrent à gauche sur le faisceau $\mathcal{O}_{\mathcal{X}^{\dagger} / R}$ leur produit opère par composition. D'autre part le degré de la réduction modulo $I^{s}, s \geq 1$ du produit est majoré par la somme des degrés. Le produit à donc la propriété du degré. Le faisceau $\mathcal{D}_{\mathcal{X}^{\dagger} / R}^{\dagger, h}$ est bien un sous-faisceau d'anneaux des cinq autres faisceaux du diagramme précédent.

Remarque 2.6.8. On peut considérer dans le cas formel un faisceau intermédiaire $\mathcal{D}_{\mathcal{X}^{\dagger} / R}^{\dagger, h} \subset \mathcal{D}_{\mathcal{X} / R}^{\dagger, h} \subset \mathcal{D}_{\mathcal{X} / R}^{\wedge, h}$ en imposant aux sections du faisceau $\mathcal{D}_{\mathcal{X} / R}^{\dagger, h}$ la condition du degré par réduction modulo les puissances de $I$. La filtration par les faisceaux $\mathcal{D}_{\mathcal{X} / R}^{\dagger, h}, h \geq 0$, du faisceau $\mathcal{D}_{\mathcal{X} / R}^{\dagger}$ est encore exhaustive.

Notation 2.6.9. Pour un ouvert $U$ †-adique affine d'algèbre $A^{\dagger}$, on note dans cet article pour simplifier

$$
D_{A^{\dagger} / R}^{h}, \quad D_{A^{\dagger} / R}, \quad D_{A^{\dagger} / R}^{\dagger, h}, \quad D_{A^{\dagger} / R}^{\dagger}
$$

pour respectivement

$$
\Gamma\left(U, \mathcal{D}_{\mathcal{X}^{\dagger} / R}^{<\infty, h}\right), \Gamma\left(U, \mathcal{D}_{\mathcal{X}^{\dagger} / R}\right), \Gamma\left(U, \mathcal{D}_{\mathcal{X}^{\dagger} / R}^{\dagger, h}\right), \Gamma\left(U, \mathcal{D}_{\mathcal{X}^{\dagger} / R}^{\dagger}\right)
$$

et

$$
D_{A^{\wedge} / R}^{h}, \quad D_{A^{\wedge} / R} \quad D_{A^{\wedge} / R}^{\dagger, h}, \quad D_{A^{\wedge} / R}^{\wedge, h}, \quad D_{A^{\wedge} / R}^{\dagger}
$$

pour respectivement

$$
\Gamma\left(U, \mathcal{D}_{\mathcal{X} / R}^{<\infty, h}\right), \quad \Gamma\left(U, \mathcal{D}_{\mathcal{X} / R}\right), \quad \Gamma\left(U, \mathcal{D}_{\mathcal{X} / R}^{\dagger, h}\right), \quad \Gamma\left(U, \mathcal{D}_{\mathcal{X} / R}^{\wedge, h}\right), \quad \Gamma\left(U, \mathcal{D}_{\mathcal{X} / R}^{\dagger}\right) .
$$

Dans le reste de cet article nous ne considérons que le cas du couple $(V, \mathfrak{m})$ où une uniformisante de $\mathfrak{m}$ n'est pas un diviseur de zéro de $V$, la condition de la proposition précédente est satisfaite et du reste n'est pas utilisée dans la définition 2.6.5.

Nous rappelons la majoration fondamentale suivante ([13, Thm. 6.4, Coro. 6.5]) que nous allons utiliser dans cet article de façon essentielle : 
Théorème 2.6.10. Soient un entier $h \geq 0, \mathcal{X}^{\dagger}=\left(X, \mathcal{O}_{\mathcal{X}^{\dagger} / V}\right)$ un schéma $\dagger$-adique lisse sur $V$, un ouvert $\dagger$-adique affine $U$ d'algèbre $A^{\dagger}$ ayant la propriété (diff), $x=\left(x_{1}, \ldots, x_{n}\right)$ un système de coordonnées au-dessus de $U$ et enfin une présentation

$$
\left(V\left[Y_{1}, \ldots, Y_{m}\right]\right)^{\dagger} \rightarrow A^{\dagger} \rightarrow 0
$$

alors il existe un entier $D(h)>0$ et un système $\Sigma$ cofinal dans l'ensemble des nombre réels $\rho>1$ tel que pour tout $\rho \in \Sigma$ il existe une une constante $C_{\rho}>0$ telle que pour tout élément $g \in A^{\dagger}$ on ait la majoration

$$
\left.\|\left(\Delta_{x}\right)^{\beta}(g)\right)\left\|_{\rho} \leq C_{\rho}\right\| g \|_{\rho} \rho^{D(h) \operatorname{ord} \beta}
$$

pour tout $\rho \in \Sigma$ assez près de 1 et pour tout élément $\beta=\left(\beta^{0}, \ldots, \beta^{h}\right) \in$ $\left(\mathbb{N}^{n}\right)^{h+1}$ où ord $\beta:=\sum_{j=0, h} p^{j}\left|\beta^{j}\right|$.

Le lecteur notera que la démonstration de la majoration précédente nécessite la continuité de la division précise à $\rho$ fixe ([13, Thm. 2.3.4]) qui est déjà à la base du théorème de l'opérateur d'un symbole total ([13, 6.1]).

\section{Le théorème du symbole total pour un opérateur différentiel $p$-adique d'échelon $h \geq 0$}

\subsection{Le théorème du symbole total}

Nous allons montrer une expression locale des opérateurs différentiels d'ordre infini et d'échelon $h \geq 0$ analogue à l'expression locale du théorème du symbole total ([11], [13]) pour les opérateurs différentiels d'ordre infini.

Définition 3.1.1. Soit un ouvert $U$ ayant la propriété (diff) d'algèbre $A^{\dagger}$, définissons l'algèbre commutative

$$
A^{\dagger}\left[\xi^{0}, \ldots, \xi^{h}\right]:=A^{\dagger}\left[\xi_{1}^{0}, \ldots, \xi_{n}^{h}\right]
$$

comme le quotient de l'algèbre des polynômes $A^{\dagger}\left[\Xi^{0}, \ldots, \Xi^{h}\right]:=A^{\dagger}\left[\Xi_{1}^{0}, \ldots, \Xi_{n}^{h}\right]$ à coefficients dans $A^{\dagger}$ par l'idéal engendré par $\left(\left(\Xi_{i}^{j}\right)^{p}-u_{j} \Xi_{i}^{j+1}, 0 \leq j \leq h-1\right.$, $1 \leq i \leq n)$ avec $u_{j}:=\frac{p^{j+1} !}{\left(p^{j !}\right)^{p}}$ et

$$
\left(A^{\dagger}\left[\xi^{0}, \ldots, \xi^{h}\right]\right)^{\dagger}
$$

son complété $\dagger$-adique pour la topologie $\mathfrak{m}$-adique de $V$. C'est donc le quotient de l'algèbre $\left(A^{\dagger}\left[\Xi^{0}, \ldots, \Xi^{h}\right]\right)^{\dagger}$ par l'idéal engendré par les mêmes éléments. 
Théorème 3.1.2. Supposant que le schéma $\mathcal{X}^{\dagger}$ est un schéma $\dagger$-adique lisse sur $V$, alors si $\left(x_{1}, \ldots, x_{n}\right)$ est un système de coordonnées locales au-dessus d'un ouvert $U$ ayant la propriété (diff) d'algèbre $A^{\dagger}$, pour tout $h \geq 0$ tout opérateur différentiel $P$ d'échelon $h$ s'écrit de manière unique au-dessus de $U$ comme une série

$$
P\left(a, \Delta, \ldots, \Delta^{p^{h}}\right)=\sum_{\beta^{0}, \ldots, \beta^{h} \in\left(\mathbb{N}_{\leq p-1}^{n}\right)^{h} \times \mathbb{N}^{n}} a_{\beta^{0}, \ldots, \beta^{h}}(\Delta)^{\beta^{0}} \ldots\left(\Delta^{p^{h}}\right)^{\beta^{h}}
$$

où $a_{\beta^{0}, \ldots, \beta^{h}}$ est une suite d'éléments de l'algèbre $A^{\dagger}$ telle que la série symbole total

$$
\sigma_{h, P}\left(a, \xi^{0}, \ldots, \xi^{h}\right):=\sum_{\beta^{0}, \ldots, \beta^{h} \in\left(\mathbb{N}_{\leq p-1}^{n}\right)^{h} \times \mathbb{N}^{n}} a_{\beta^{0}, \ldots, \beta^{h}}\left(\xi^{0}\right)^{\beta^{0}} \ldots\left(\xi^{h}\right)^{\beta^{h}}
$$

est un élément de l'algèbre $\left(A^{\dagger}\left[\xi^{0}, \ldots, \xi^{h}\right]\right)^{\dagger}$.

Démonstration. Soit $P$ un opérateur de $D_{A^{\wedge} / V}^{\wedge, h}$, alors par définition c'est une limite quand $s \rightarrow \infty$ d'une suite d'opérateurs de la forme

$$
P_{s}=P_{s}\left(a, \Delta, \ldots, \Delta^{p^{h}}\right)
$$

où chaque opérateurs $P_{s}$ est un opérateur d'ordre fini. En vertu de la proposition 2.6.3 l'opérateur $P$ s'écrit de façon unique :

$$
P\left(a, \Delta, \ldots, \Delta^{p^{h}}\right)=\sum_{\beta^{0}, \ldots, \beta^{h} \in\left(\mathbb{N}_{\leq p-1}^{n}\right)^{h} \times \mathbb{N}^{n}} a_{\beta^{0}, \ldots, \beta^{h}}(\Delta)^{\beta^{0}} \ldots\left(\Delta^{p^{h}}\right)^{\beta^{h}}
$$

où $a_{\beta^{0}, \ldots, \beta^{h}}$ est une suite d'éléments de $A^{\wedge}$ telle que $v_{\mathfrak{m}}\left(a_{\beta^{0}, \ldots, \beta^{h}}\right) \rightarrow \infty$ quand $\left|\beta^{h}\right| \rightarrow \infty$.

L'application rep qui à $\alpha \in \mathbb{N}^{n}$ associe

$$
\operatorname{rep}(\alpha):=\left(\beta^{0}, \ldots, \beta^{h}\right) \in\left(\mathbb{N}_{\leq p-1}^{n}\right)^{h} \times \mathbb{N}^{n}
$$

tels que $\alpha_{i}=\beta_{i}^{0}+\cdots+\beta_{i}^{h} p^{h}, 0 \leq \beta_{i}^{j} \leq p-1,0 \leq j \leq h-1$ est une bijection. On a alors l'égalité

$\sum_{\beta^{0}, \ldots, \beta^{h} \in\left(\mathbb{N}_{\leq p-1}^{n}\right)^{h} \times \mathbb{N}^{n}} a_{\beta^{0}, \ldots, \beta^{h}}(\Delta)^{\beta^{0}} \ldots\left(\Delta^{p^{h}}\right)^{\beta^{h}}=\sum_{\alpha \in \mathbb{N}^{n}} a_{r e p(\alpha)} \frac{\alpha !}{(p !)^{\left|\beta^{1}\right|} \ldots\left(p^{h !}\right)^{\left|\beta^{h}\right|}} \Delta^{\alpha}$.

Mais par hypothèse $P$ est une section de $\mathcal{D}_{\mathcal{X}^{\dagger} / V}^{\dagger}$ et le théorème du symbole total d'un opérateur différentiel $p$-adique ([13, Thm. 5.1]) montre que l'on a l'égalité

$a_{\beta^{0}, \ldots, \beta^{h}} \frac{\left(\beta^{0}+\cdots+\beta^{h} p^{h}\right) !}{(p !)^{\left|\beta^{1}\right|} \cdots\left(p^{h !}\right)^{\left|\beta^{h}\right|}}=\sum_{0 \leq k \leq \beta^{0}+\cdots+\beta^{h} p^{h}}\left(\begin{array}{l}\beta^{0}+\cdots+\beta^{h} p^{h} \\ k\end{array}\right)(-x)^{k} P\left(x^{\beta^{0}+\cdots+\beta^{h} p^{h}-k}\right)$

en particulier les coefficients $a_{\beta^{0}, \ldots, \beta^{h}}$ appartiennent à l'algèbre $A^{\dagger}=A_{K}^{\dagger} \cap A^{\wedge}$. 
Nous reprenons la démonstration de la majoration du théorème du symbole total d'un opérateur différentiel $p$-adique de l'article [13]. Soit

$$
0 \rightarrow J \rightarrow\left(V\left[Y_{1}, \ldots, Y_{m}\right]\right)^{\dagger} \rightarrow A^{\dagger} \rightarrow 0
$$

une présentation de l'algèbre $A^{\dagger}$. Il existe un nombre $\rho>1$ tel que $x_{1}, \ldots, x_{n}$ appartiennent à l'algèbre $A_{K, \rho}^{\dagger}$ et donc $x^{\alpha}$ appartient à $A_{K, \rho}^{\dagger}$ pour tout $\alpha \in \mathbb{N}^{n}$. En vertu du corollaire 3.2.1 de l'article [13] il existe un nombre $\rho^{\prime}, 1<\rho^{\prime} \leq \rho$ tel que $P\left(x^{\beta^{0}+\cdots+\beta^{h} p^{h}-k}\right)$ appartient à $A_{K, \rho^{\prime}}^{\dagger}$ pour tout $\beta^{0}+\cdots+\beta^{h} p^{h}$. En particulier les coefficients $a_{\beta^{0}, \ldots, \beta^{h}}$ appartiennent à $A_{K, \rho^{\prime}}^{\dagger}$ pour tout $\beta^{0}, \ldots, \beta^{h} \in$ $\left(\mathbb{N}_{\leq p-1}^{n}\right)^{h} \times \mathbb{N}^{n}$. Reste à calculer leurs normes.

Le raisonnement de la démonstration du lemme 5.2 de l'article [13] montre que la condition sur le degré de la définition 2.6.5 entraîne l'existence de deux constantes $C^{\prime}>0,0<\lambda<1$ telles que l'on ait la majoration pour tout $\beta^{0}, \ldots, \beta^{h}$ :

$$
(* *)_{h} \quad\left\|a_{\beta^{0}, \ldots, \beta^{h}}\right\|_{1} \leq C^{\prime} \lambda^{\left|\beta^{0}\right|+\cdots+\left|\beta^{h}\right| p^{h}} .
$$

De plus en vertu de la formule donnant les coefficients de $P$ à l'aide de l'action de $P$ sur les monômes en $x$ et du corollaire 3.2.1 de l'article [13] il existe un nombre $\rho>1$ et une constante $C^{\prime \prime}>0$ tels que l'on ait la majoration

$$
\left.(* * *)_{h} \quad \quad\left\|a_{\beta^{0}, \ldots, \beta^{h}} \frac{\left(\beta^{0}+\cdots+\beta^{h} p^{h}\right) !}{(p !)^{\left|\beta^{1}\right|} \ldots\left(p^{h !}\right)^{\left|\beta^{h}\right|}}\right\|_{\rho} \leq C^{\prime \prime} \rho^{l\left(\left|\beta^{0}\right|+\cdots+\left|\beta^{h}\right| p^{h}\right.}\right)
$$

pour un entier $l \geq 1$.

Mais ces deux majorations $(* *)_{h},(* * *)_{h}$ n'impliquent pas en faisant le produit le théorème 3.1 .2 comme les majorations analogues $(* *),(* * *)$ du $\S 5$ de l'article [13] impliquaient le théorème du symbole total d'un opérateur différentiel $p$-adique ([13, Thm. 5.1]) à cause du facteur non trivial du point de vue $p$-adique

$$
\frac{\left(\beta^{0}+\cdots+\beta^{h} p^{h}\right) !}{(p !)^{\left|\beta^{1}\right|} \cdots\left(p^{h !}\right)^{\left|\beta^{h}\right|}}
$$

Aussi la situation est plus délicate et il faut un autre argument qui utilise l'action à gauche de $\mathcal{D}_{\mathcal{X}^{\dagger} / V}^{\dagger, h} \operatorname{sur} \mathcal{O}_{\mathcal{X}^{\dagger} / V}$.

Lemme 3.1.3. Pour tout élément $\theta \in V,|\theta|<\omega:=|\pi|, \pi^{p-1}+p=0$, il existe un nombre $\rho>1$ tel que

$$
\lim _{\left|\beta^{h}\right| \rightarrow \infty}\left\|(\theta)^{\left|\beta^{0}\right|} \ldots\left(\theta^{p^{h}} / p^{h} !\right)^{\left|\beta^{h}\right|} a_{\beta^{0}, \ldots, \beta^{h}}\right\|_{\rho}=0 .
$$


Démonstration. En vertu du théorème 6.7 de l'article [13] pour toute élément $f$ de $A^{\dagger}$ il existe un nombre $\rho>1$ tel que

$$
\begin{gathered}
\lim _{\left|\beta^{h}\right| \rightarrow \infty}\left\|a_{\beta^{0}, \ldots, \beta^{h}}(\Delta)^{\beta^{0}} \ldots\left(\Delta^{p^{h}}\right)^{\beta^{h}}(f)\right\|_{\rho}= \\
\lim _{\left|\beta^{h}\right| \rightarrow \infty}\left\|a_{\beta^{0}, \ldots, \beta^{h}} \frac{\left(\beta^{0}+\cdots+\beta^{h} p^{h}\right) !}{(p !)^{\left|\beta^{1}\right|} \ldots\left(p^{h !}\right)^{\left|\beta^{h}\right|}} \Delta^{\beta^{0}+\cdots+\beta^{h} p^{h}}(f)\right\|_{\rho}=0 .
\end{gathered}
$$

Appliquons ceci à la l'élément $f=\exp \left(\theta\left(x_{1}+\cdots+x_{n}\right)\right)$ qui pour $|\theta|<\omega$ est un élément de $A^{\dagger}$. On trouve un nombre $\rho>1$ tel que :

$$
\lim _{\left|\beta^{h}\right| \rightarrow \infty}\left\|(\theta)^{\left|\beta^{0}\right|} \ldots\left(\theta^{p^{h}} / p^{h} !\right)^{\left|\beta^{h}\right|} a_{\beta^{0}, \ldots, \beta^{h}} \exp \left(\theta\left(x_{1}+\cdots+x_{n}\right)\right)\right\|_{\rho}=0 .
$$

Mais

$$
\begin{aligned}
&\left\|(\theta)^{\left|\beta^{0}\right|} \ldots\left(\theta^{p^{h}} / p^{h} !\right)^{\left|\beta^{h}\right|} a_{\beta^{0}, \ldots, \beta^{h}}\right\|_{\rho} \leq \\
& \leq\left\|(\theta)^{\left|\beta^{0}\right|} \ldots\left(\theta^{p^{h}} / p^{h !}\right)^{\left|\beta^{h}\right|} a_{\beta^{0}, \ldots, \beta^{h}} \exp \left(\theta\left(x_{1}+\cdots+x_{n}\right)\right)\right\|_{\rho} \\
& \quad\left\|\exp \left(-\theta\left(x_{1}+\cdots+x_{n}\right)\right)\right\|_{\rho} .
\end{aligned}
$$

D'où l'existence pour tout nombre $\theta,|\theta|<\omega$ d'un nombre $\rho>1$ tel que :

$$
\lim _{\left|\beta^{h}\right| \rightarrow \infty}\left\|(\theta)^{\left|\beta^{0}\right|} \ldots\left(\theta^{p^{h}} / p^{h} !\right)^{\left|\beta^{h}\right|} a_{\beta^{0}, \ldots, \beta^{h}}\right\|_{\rho}=0 .
$$

Considérons la rétraction reste du théorème de division $([13,2.3 .2])$ par une base $B$ de division de l'idéal $J$ :

$$
\operatorname{Retr}_{\mathrm{B}}: A_{K}^{\wedge} \rightarrow\left(K\left[Y_{1}, \ldots, Y_{m}\right]\right)^{\dagger}
$$

qui est continue pour la norme $\|-\|_{1}$. En vertu de la majoration $(* *)_{h}$ il existe une constante $C_{1}>0$ telle que

$$
\left(* *_{h}^{\prime}\right) \quad \quad\left\|\operatorname{Retr}_{\mathrm{B}}\left(a_{\beta^{0}, \ldots, \beta^{h}}\right)\right\|_{1} \leq C_{1} \lambda^{\left|\beta^{0}\right|+\cdots+\left|\beta^{h}\right| p^{h}}
$$

pour tout $\beta^{0}, \ldots, \beta^{h}$.

En vertu du théorème $([13,2.3 .4])$ il existe un système cofinal de nombre $\rho>1$ assez près de 1 tel que pour tout $\rho$ appartenant à ce système la rétraction reste du théorème de division par une base $B$ de division de l'idéal $J$ :

$$
\operatorname{Retr}_{\mathrm{B}}: A_{K, \rho}^{\dagger} \rightarrow\left(V\left[Y_{1}, \ldots, Y_{m}\right]\right)_{K, \rho}^{\dagger}
$$

qui est continue pour la norme $\|-\|_{\rho}$. En vertu de la majoration $(* * *)_{h}$ il existe un nombre $\rho^{\prime}>1$ et une constante $C_{2}$ tels que l'on ait les majorations :

$\left(* * *^{\prime}\right)_{h} \quad\left\|\operatorname{Retr}_{\mathrm{B}}\left(a_{\beta^{0}, \ldots, \beta^{h}}\right) \frac{\left(\beta^{0}+\cdots+\beta^{h} p^{h}\right) !}{(p !)^{\left|\beta^{1}\right|} \ldots\left(p^{h} !\right)^{\left|\beta^{h}\right|}}\right\|_{\rho^{\prime}} \leq C_{2} \rho^{l\left(\left|\beta^{0}\right|+\cdots+\left|\beta^{h}\right| p^{h}\right)}$

pour tout $\beta^{0}, \ldots, \beta^{h} \in\left(\mathbb{N}_{\leq p-1}^{n}\right)^{h} \times \mathbb{N}^{n}$. 
Finalement en vertu du lemme précédent il existe un nombre $\rho^{\prime}>1$ tel que l'on ait l'égalité

$$
(* * * *)_{h} \quad \lim _{\left|\beta^{h}\right| \rightarrow \infty}\left\|(\theta)^{\left|\beta^{0}\right|} \ldots\left(\theta^{\mathrm{p}^{\mathrm{h}}} / \mathrm{p}^{\mathrm{h}} !\right)^{\left|\beta^{\mathrm{h}}\right|} \operatorname{Retr}_{\mathrm{B}}\left(\mathrm{a}_{\beta^{0}, \ldots, \beta^{\mathrm{h}}}\right)\right\|_{\rho^{\prime}}=0 .
$$

Si on écrit $\operatorname{Retr}_{\mathrm{B}}\left(a_{\beta^{0}, \ldots, \beta^{h}}\right)(Y)=\sum_{\beta \in\left(\mathbb{N}^{n}\right)_{\leq p-1}^{h} \times \mathbb{N}^{n}, \gamma \in \mathbb{N}^{m}} a_{\beta, \gamma} Y^{\gamma}$ les majorations $\left(* *^{\prime}\right)_{h}$ donnent les majorations

$$
\left|a_{\beta, \gamma}\right| \leq C_{1} \lambda^{\beta^{0}+\cdots+\beta^{h} p^{h}}
$$

pour tous $\beta, \gamma \in\left(\mathbb{N}_{\leq p-1}^{n}\right)^{h} \times \mathbb{N}^{n} \times \mathbb{N}^{m}$ et les majorations $\left(* * *^{\prime}\right)_{h}$ donnent les majorations

$$
\left|a_{\beta, \gamma}\right| \leq C_{2} \frac{\left|(p !)^{\left|\beta^{1}\right|} \ldots\left(p^{h} !\right)^{\left|\beta^{h}\right|}\right|}{\left|\left(\beta^{0}+\cdots+\beta^{h} p^{h}\right) !\right|} \rho^{\prime-|\gamma|} \rho^{l\left(\left|\beta^{0}\right|+\cdots+\left|\beta^{h}\right| p^{h}\right)}
$$

pour tous $\beta, \gamma \in\left(\mathbb{N}_{\leq p-1}^{n}\right)^{h} \times \mathbb{N}^{n} \times \mathbb{N}^{m}$. Choisissons un nombre $\lambda^{\prime}, 0<\lambda^{\prime}<\omega$ et posons

$$
\operatorname{Retr}_{\mathrm{B}}\left(\mathrm{a}_{\beta^{0}, \ldots, \beta^{\mathrm{h}}}\right)(\mathrm{Y})=\mathrm{a}_{\beta}^{\prime}(\mathrm{Y})+\mathrm{a}_{\beta}^{\prime \prime}(\mathrm{Y})
$$

où

$$
\begin{aligned}
& a_{\beta}^{\prime}(Y):=\sum_{\beta \in\left(\mathbb{N}^{n}\right)_{\leq p-1}^{h} \times \mathbb{N}^{n}, \gamma \in \mathbb{N}^{m},\left|a_{\beta, \gamma}\right|<\lambda^{\prime\left|\beta^{0}\right|+\cdots+\left|\beta^{h}\right| p^{h}}} a_{\beta, \gamma} Y^{\gamma} \\
& a_{\beta}^{\prime \prime}(Y):=\sum_{\beta \in\left(\mathbb{N}^{n}\right)_{\leq p-1}^{h} \times \mathbb{N}^{n}, \gamma \in \mathbb{N}^{m},\left|a_{\beta, \gamma}\right| \geq \lambda^{\prime\left|\beta^{0}\right|+\cdots+\left|\beta^{h}\right| p^{h}}} a_{\beta, \gamma} Y^{\gamma}
\end{aligned}
$$

En faisant le produit des deux types de majorations précédentes des coefficients $a_{\beta, \gamma}$ on trouve les majorations :

$(\bullet)_{h}\left|a_{\beta, \gamma}\right| \leq C_{2}^{1 / 2} \rho^{\prime-|\gamma| / 2} \lambda^{l\left(\left|\beta^{0}\right|+\cdots+\left|\beta^{h}\right| p^{h}\right) / 2} \rho^{l\left(\left|\beta^{0}\right|+\cdots+\left|\beta^{h}\right| p^{h}\right) / 2} \omega^{-\left(\left|\beta^{0}\right|+\cdots+\left|\beta^{h}\right|\right) / 2}$

pour tous $\beta, \gamma \in\left(\mathbb{N}_{\leq p-1}^{n}\right)^{h} \times \mathbb{N}^{n} \times \mathbb{N}^{m}$ tels que $\left|a_{\beta, \gamma}\right|<\lambda^{\prime\left|\beta^{0}\right|+\cdots+\left|\beta^{h}\right| p^{h}}$.

Si $\left|a_{\beta, \gamma}\right| \geq \lambda^{\prime\left|\beta^{0}\right|+\cdots+\left|\beta^{h}\right| p^{h}}$ les majorations $\left(* * *^{\prime}\right)_{h}$ sont insuffisantes aussi nous allons utiliser l'égalité $(* * * *)_{h}$. Posons pour $\rho^{\prime}>1$ assez près de 1

$$
\| a_{\beta}^{\prime \prime}||_{\rho^{\prime}}:=\max _{\gamma \in \mathbb{N}^{m}}\left|a_{\beta, \gamma}\right| \rho^{\prime|\gamma|}=\left|a_{\beta, \gamma\left(\beta, \rho^{\prime}\right)}\right| \rho^{\prime\left|\gamma\left(\beta, \rho^{\prime}\right)\right|} .
$$

L'égalité $(* * * *)_{h}$ montre que

$$
\lim _{\left|\beta^{h}\right| \rightarrow \infty}\left|a_{\beta, \gamma\left(\beta, \rho^{\prime}\right)}\right| \rho^{\prime\left|\gamma\left(\beta, \rho^{\prime}\right)\right|}|\theta|^{\left(\left|\beta^{0}\right|+\cdots+\left|\beta^{h}\right| p^{h}\right)} \omega^{-\left(\left|\beta^{1}\right|(p-1)+\cdots+\left|\beta^{h}\right|\left(p^{h}-1\right)\right)}=0 .
$$


Mais comme $\left|a_{\beta, \gamma}\right| \geq \lambda^{\prime\left|\beta^{0}\right|+\cdots+\left|\beta^{h}\right| p^{h}}$ ceci entraîne que

$$
\gamma(\beta, \rho) \leq \frac{\log \left(1 / \lambda^{\prime}|\theta|\right)}{\log \rho^{\prime}}\left(\left|\beta^{0}\right|+\cdots+\left|\beta^{h}\right| p^{h}\right)
$$

pour $\left|\beta^{h}\right|$ assez grand. Si $1<\rho^{\prime}<\rho_{0}$ on a $\gamma\left(\beta, \rho^{\prime}\right) \leq \gamma\left(\beta, \rho_{0}\right)$. Ceci entraîne que pour tout $\rho^{\prime}, 1<\rho^{\prime}<\rho_{0}$

$$
\gamma\left(\beta, \rho^{\prime}\right) \leq \frac{\log \left(1 / \lambda^{\prime}|\theta|\right)}{\log \rho_{0}}\left(\left|\beta^{0}\right|+\cdots+\left|\beta^{h}\right| p^{h}\right) .
$$

D'où une constante $l^{\prime}$ telle que

$$
\left|a_{\beta, \gamma}\right| \leq C_{3} \rho^{\prime-|\gamma|} \rho^{\prime l^{\prime}\left(\left|\beta^{0}\right|+\cdots+\left|\beta^{h}\right| p^{h}\right)}
$$

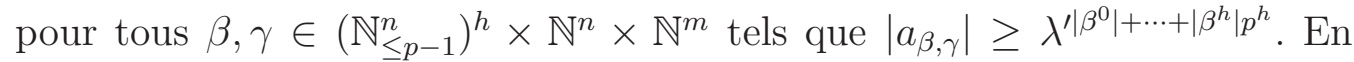
faisant le produit de ces majorations avec les majorations

$$
\left|a_{\beta, \gamma}\right| \leq C_{1} \lambda^{\beta^{0}+\cdots+\beta^{h} p^{h}}
$$

on trouve les majorations

$$
(\diamond)_{h} \quad\left|a_{\beta, \gamma}\right| \leq C_{1}^{1 / 2} C_{3}^{1 / 2} \rho^{\prime-|\gamma| / 2} \lambda^{\left(\left|\beta^{0}\right|+\cdots+\left|\beta^{h}\right| p^{h}\right) / 2} \rho^{l^{\prime}\left(\left|\beta^{0}\right|+\cdots+\left|\beta^{h}\right| p^{h}\right) / 2}
$$

pour tout $\rho^{\prime}>1$ assez près de 1 . Les majorations $(\boldsymbol{\vee})_{h}$ et $(\diamond \bullet)_{h}$ montrent que la série

$$
\sum_{\beta \in\left(\mathbb{N}_{\leq p-1}^{n}\right)^{h} \times \mathbb{N}^{n}, \gamma \in \mathbb{N}^{m}} a_{\beta, \gamma} Y^{\gamma}\left(\Xi^{0}\right)^{\beta^{0}} \ldots\left(\Xi^{h}\right)^{\beta^{h}}
$$

admet un rayon de convergence strictement plus grand que 1 par rapport à toutes les variables. Sa classe dans l'algèbre $\left(A^{\dagger}\left[\xi^{0}, \ldots, \xi^{h}\right]\right)^{\dagger}$ est égale au symbole total

$$
P\left(a, \xi^{0}, \ldots, \xi^{h}\right)=\sum_{\beta^{0}, \ldots, \beta^{h} \in\left(\mathbb{N}_{\leq p-1}^{n}\right)^{h} \times \mathbb{N}^{n}} a_{\beta^{0}, \ldots, \beta^{h}}\left(\xi^{0}\right)^{\beta^{0}} \ldots\left(\xi^{h}\right)^{\beta^{h}}
$$

qui est donc un élément de l'algèbre $\left(A^{\dagger}\left[\xi^{0}, \ldots, \xi^{h}\right]\right)^{\dagger}$. D'où le théorème 3.1.2.

Pour tout $h \geq 0$ il existe en particulier un nombre $\lambda, 0<\lambda<1$ et une constante $C>0$ tels que pour un nombre $\rho>1$ assez près de 1 on a la majoration :

$$
\left\|a_{\beta^{0}, \ldots, \beta^{h}}\right\|_{\rho} \leq C \lambda^{\left|\beta^{0}\right|+\cdots+\left|\beta^{h}\right| p^{h}}
$$

pour tout $\beta^{0}, \ldots, \beta^{h} \in\left(\mathbb{N}_{\leq p-1}^{n}\right)^{h} \times \mathbb{N}^{n}$. Réciproquement : 
Théorème 3.1.4. Supposant que le schéma $\dagger$-adique $\left(X, \mathcal{O}_{\mathcal{X}^{\dagger} / V}\right)$ est lisse sur $V$, alors si $\left(x_{1}, \ldots, x_{n}\right)$ est un système de coordonnées locales au-dessus d'un ouvert $U$ ayant la propriété (diff) d'algèbre $A^{\dagger}$, pour tout $h \geq 0$ soit $a_{\beta^{0}, \ldots, \beta^{h}}$ une suite d'éléments de l'algèbre $A^{\dagger}$ telle que la série

$$
P\left(a, \xi^{0}, \ldots, \xi^{h}\right)=\sum_{\beta^{0}, \ldots, \beta^{h} \in\left(\mathbb{N}_{\leq p-1}^{n}\right)^{h} \times \mathbb{N}^{n}} a_{\beta^{0}, \ldots, \beta^{h}}\left(\xi^{0}\right)^{\beta^{0}} \ldots\left(\xi^{h}\right)^{\beta^{h}}
$$

est un élément de l'algèbre $\left(A^{\dagger}\left[\xi^{0}, \ldots, \xi^{h}\right]\right)^{\dagger}$. Alors la série

$$
P\left(a, \Delta, \ldots, \Delta^{p^{h}}\right)=\sum_{\beta^{0}, \ldots, \beta^{h} \in\left(\mathbb{N}_{\leq p-1}^{n}\right)^{h} \times \mathbb{N}^{n}} a_{\beta^{0}, \ldots, \beta^{h}}(\Delta)^{\beta^{0}} \ldots\left(\Delta^{p^{h}}\right)^{\beta^{h}}
$$

est un opérateur différentiel p-adique d'ordre infini et d'échelon $h$.

Démonstration. La série $P\left(a, \Delta, \ldots, \Delta^{p^{h}}\right)$ a la condition du degré de la définition 2.6.5. Il suffit de montrer que $P\left(a, \Delta, \ldots, \Delta^{p^{h}}\right)$ opère sur l'algèbre $A^{\dagger}$. Mais ceci est une conséquence de la majoration du théorème 2.6.10.

Exemple 3.1.5. Soit $V\left[x_{1}, \ldots, x_{n}\right]$ l'algèbre des polynômes à $n$-variables à coefficients dans $V$, son complété †-adique $\left(V\left[x_{1}, \ldots, x_{n}\right]\right)^{\dagger}$ et

$$
\mathcal{A}_{n}^{\dagger}:=\left(\operatorname{Spec} k\left[x_{1}, \ldots, x_{n}\right], \mathcal{O}_{\mathcal{A}_{n}^{\dagger} / V}\right)
$$

le schéma †-affine associé.

Soit un ouvert principal $U$ défini par un polynôme $\bar{f}$, alors la $V$-algèbre des sections $\Gamma\left(U, \mathcal{O}_{\mathcal{A}_{n}^{\dagger} / V}\right)$ est l'algèbre $\left(V\left[x_{1}, \ldots, x_{n}, 1 / f\right]\right)^{\dagger}$ pour n'importe quel relèvement $f$ de $\bar{f}$ [15].

Le théorème 3.1.2, du symbol total pour les opérateurs différentiels $p$ adiques d'échelon 0 , dit qu'une section au-dessus de $U$ du faisceau $\mathcal{D}_{\mathcal{A}_{n}^{\dagger} / V}^{\dagger, 0}$ est une somme infini

$$
\sum_{\alpha \in \mathbb{N}^{n}, \gamma \in \mathbb{N}, \beta \in \mathbb{N}^{n}} a_{\alpha, \gamma, \beta} x^{\alpha} f^{-\gamma}\left(\Delta_{x}^{1}\right)^{\beta}, a_{\alpha, \gamma, \beta} \in V
$$

telle que la série entière $\sum_{\alpha \in \mathbb{N}^{n}, \gamma \in \mathbb{N}, \beta \in \mathbb{N}^{n}} a_{\alpha, \gamma, \beta} x^{\alpha} T^{\gamma}\left(\Xi^{0}\right)^{\beta}$ appartient à l'algèbre commutative $\left(V\left[x, T, \Xi^{0}\right]\right)^{\dagger}$ pour $\operatorname{deg}(\bar{f})>0$.

Et réciproquement le théorème 3.1.4 dit qu'une telle somme définit une section au-dessus de $U$ du faisceau $\mathcal{D}_{\mathcal{A}_{n}^{\dagger} / V}^{\dagger, 0} \operatorname{pour} \operatorname{deg}(\bar{f})>0$.

Pour $\operatorname{deg}(\bar{f})=0$ qui correspond au cas $U=\operatorname{Spec} k\left[x_{1} \ldots, x_{n}\right]$ on a le même résultat en ignorant $f, \gamma, T$. 
Proposition 3.1.6. Soit $\mathcal{X}^{\dagger}:=\left(X, \mathcal{O}_{\mathcal{X}^{\dagger} / V}\right)$ un schéma $\dagger$-adique lisse sur $V$, alors les faisceaux $\mathcal{D}_{\mathcal{X}^{\dagger} / V}^{\dagger, h}, h \in \mathbb{N}$, constituent une filtration du faisceau $\mathcal{D}_{\mathcal{X}^{\dagger} / V}^{\dagger}$ croissante exhaustive par des faisceaux de $\mathcal{O}_{\mathcal{X}^{\dagger} / V^{-a l g e ̀ b r e s . ~}}$

Démonstration. Par définition les faisceaux $\mathcal{D}_{\mathcal{X}^{\dagger} / V}^{\dagger, h}$ sont des sous-faisceaux de $\mathcal{O}_{\mathcal{X}^{\dagger} / V^{-a l g e ̀ b r e s ~ d u ~ f a i s c e a u ~}} \mathcal{D}_{\mathcal{X}^{\dagger} / V}^{\dagger}$. Il suffit de montrer que la filtration par les échelons est exhaustive. La question est locale, il suffit de montrer que localement tout opérateur d'ordre infini appartient à $\mathcal{D}_{\mathcal{X}^{\dagger} / V}^{\dagger, h}$ pour un $h \geq 0$ non précisé.

Soit $x=\left(x_{1}, \ldots, x_{n}\right)$ un système de coordonnées locales au-dessus d'un ouvert $U$ ayant la propriété (diff) d'algèbre $A^{\dagger}$, alors en vertu du théorème du symbole total ([13, Thm. 5.1]) tout opérateur différentiel $P(x, \Delta) p$-adique s'écrit de manière unique

$$
P(x, \Delta)=\sum_{\alpha \in \mathbb{N}^{n}} a_{\alpha} \Delta^{\alpha}
$$

où $a_{\alpha}$ est une suite d'éléments de l'algèbre $A^{\dagger}$. De plus si

$$
\left(V\left[Y_{1}, \ldots, Y_{m}\right]\right)^{\dagger} \rightarrow A^{\dagger} \rightarrow 0
$$

une présentation de l'algèbre $A^{\dagger}$, en vertu du lemme 5.2 de [13] il existe deux constantes $C>0,0<\lambda<1$ telles que l'on ait la majoration :

$$
\left\|a_{\alpha}\right\|_{1} \leq C \lambda^{|\alpha|} .
$$

Choisissons un entier $h \geq 0$ tel que $\lambda<p^{-1 / p^{h}(p-1)}<1$. Pour $\alpha=\left(\alpha_{1}, \ldots, \alpha_{n}\right)$ soit $\alpha_{i}=a_{i, 0}+\cdots+a_{i, n} p^{h}$ la division de $\alpha_{i}$ par $p^{h}$. Posons

$$
\begin{gathered}
\beta^{0}:=\left(a_{1,0}+\cdots+a_{n, 0}\right), \ldots \beta^{h}:=\left(a_{1, h}+\cdots+a_{n, h}\right), \\
a_{\alpha} \Delta^{\alpha}=a_{\alpha} \frac{(p !)^{\left|\beta^{1}\right|} \ldots\left(p^{h} !\right)^{\left|\beta^{h}\right|}}{\alpha !}(\Delta)^{\beta^{0}} \ldots\left(\Delta^{p^{h}}\right)^{\beta^{h}} .
\end{gathered}
$$

Mais il existe deux constante $\mu_{1}>0, \mu_{2}$ telles que

$$
v\left(a_{\alpha}\right)+v\left(\frac{(p !)^{\left|\beta^{1}\right|} \ldots\left(p^{h} !\right)^{\left|\beta^{h}\right|}}{\alpha !}\right) \geq \mu_{1}|\alpha|+\mu_{2}
$$

où $v(a):=v_{\mathfrak{m}}(a)$, qui montre que les opérateurs $a_{\alpha} \Delta^{\alpha}$ sont d'échelon $h$ à l'exception éventuelle d'un nombre fini d'entre eux. Ceci montre que l'opérateur $P(x, \Delta)$ est d'échelon $h \geq 0$ pour $h$ assez grand non précisé.

Remarque 3.1.7. La même démonstration montre que la filtration $\mathcal{D}_{\mathcal{X} / V}^{\wedge, h}$, $h \geq 0$ est une filtration croissante exhaustive du faisceau $\mathcal{D}_{\mathcal{X} / V}^{\dagger}$ ainsi que la filtration $\mathcal{D}_{\mathcal{X} / V}^{\dagger, h}, h \geq 0$. 


\subsection{Trivialité cohomologique du faisceau des opérateurs différen- tiels d'échelon $h$}

Corollaire 3.2.1. Supposant que le schéma $\mathcal{X}^{\dagger}$ est $\dagger$-adique lisse sur $V$. Alors si $\left(x_{1}, \ldots, x_{n}\right)$ est un système de coordonnées locales au-dessus d'un ouvert $U$ ayant la propriété (diff) d'algèbre $A^{\dagger}$ l'application symbole total qui à un opérateur différentiel d'échelon $h \geq 0$

$$
P\left(a, \Delta, \ldots, \Delta^{p^{h}}\right)=\sum_{\beta^{0}, \ldots, \beta^{h} \in\left(\mathbb{N}_{\leq p-1}^{n}\right)^{h} \times \mathbb{N}^{n}} a_{\beta^{0}, \ldots, \beta^{h}}(\Delta)^{\beta^{0}} \ldots\left(\Delta^{p^{h}}\right)^{\beta^{h}}
$$

associe la série symbole total

$$
\sigma_{h, P}\left(a, \xi^{0}, \ldots, \xi^{h}\right):=\sum_{\beta^{0}, \ldots, \beta^{h} \in\left(\mathbb{N}_{\leq p-1}^{n}\right)^{h} \times \mathbb{N}^{n}} a_{\beta^{0}, \ldots, \beta^{h}}\left(\xi^{0}\right)^{\beta^{0}} \ldots\left(\xi^{h}\right)^{\beta^{h}}
$$

est un isomorphisme de $A^{\dagger}$-modules à gauche entre $D_{A^{\dagger} / V}^{\dagger, h}$ et $\left(A^{\dagger}\left[\xi^{0}, \ldots, \xi^{h}\right]\right)^{\dagger}$.

Démonstration. C'est une conséquence des théorèmes 3.1.2 et 3.1.4.

Bien entendu l'isomorphisme du symbole total dépend des coordonnées et ne respecte pas la structure multiplicative.

Notons $\mathcal{Y}^{\dagger}$ le schéma $\dagger$-adique affine associé à $\left(A^{\dagger}\left[\xi^{0}, \ldots, \xi^{h}\right]\right)^{\dagger}$ et $q$ désigne la projection naturelle de $Y:=\operatorname{Spec} A\left[\xi^{0}, \ldots, \xi^{h}\right]$ sur $U$.

Corollaire 3.2.2. Sous les hypothèses du corollaire précédent pour tout $h \geq 0$ l'isomorphisme du corollaire précédent se localise en un isomorphisme

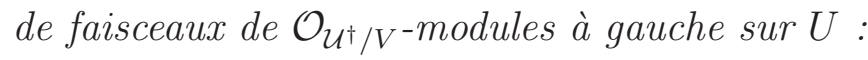

$$
\mathcal{D}_{\mathcal{U}^{\dagger} / V}^{\dagger, h} \simeq q_{*} \mathcal{O}_{\mathcal{Y}^{\dagger} / V}
$$

Démonstration. Soit $W$ un ouvert principal de $U$ d'algèbre $A_{f}^{\dagger}$ alors les différentielles $\left(d x_{1}, \ldots, d x_{n}\right)$ forme encore une base au-dessus de $W \mathrm{du} \mathcal{O}_{\mathcal{X}^{\dagger} / V^{-}}$ module des formes différentielles séparées $\Omega_{\mathcal{X}^{\dagger} / V}$. D'où en vertu du corollaire précédent un isomorphisme

$$
\Gamma\left(W, \mathcal{D}_{\mathcal{U}^{\dagger} / V}^{\dagger, h}\right) \simeq\left(A_{f}^{\dagger}\left[\xi^{0}, \ldots, \xi^{h}\right]\right)^{\dagger}
$$

qui est compatible aux restrictions. D'où le corollaire. 
Théorème 3.2.3. Supposons que $\mathcal{X}^{\dagger}$ est $\dagger$-adique lisse sur $V$. Alors si $U$ est un ouvert $U$ ayant la propriété (diff) le faisceau $\mathcal{D}_{\mathcal{U}^{\dagger} / V}^{\dagger, h}$ est acyclique au-dessus de $U$ :

$$
\mathrm{H}^{\mathrm{i}}\left(\mathrm{U}, \mathcal{D}_{\mathcal{U}^{\dagger} / \mathrm{V}}^{\dagger, \mathrm{h}}\right)=0
$$

pour $i \geq 1$.

Démonstration. Soit $\left(x_{1}, \ldots, x_{n}\right)$ est un système de coordonnées locales au-dessus d'un, considérons la projection $\left.q: Y:=\operatorname{Spec} A\left[\xi^{0}, \ldots, \xi^{h}\right]\right) \rightarrow U$, si $W$ est un ouvert affine principal de $U$ son image inverse $q^{-1} W$ est un ouvert affine principal. En vertu du théorème d'acyclicité [15] la cohomologie $\mathrm{H}^{\mathrm{i}}\left(\mathrm{q}^{-1} \mathrm{~W}, \mathcal{O}_{\mathcal{Y}^{\dagger} / \mathrm{V}}\right)$ est nulle pour $i \geq 1$ et donc les faisceaux images directes supérieures $R^{i} q_{*} \mathcal{O}_{\mathcal{Y}^{\dagger} / V}$ sont nuls pour $i \geq 1$. On a alors les isomorphismes :

$$
\mathbf{R} \Gamma\left(Y, \mathcal{O}_{\mathcal{Y}^{\dagger} / V}\right) \simeq \mathbf{R} \Gamma\left(U, q_{*} \mathcal{O}_{\mathcal{Y}^{\dagger} / V}\right) \simeq \mathbf{R} \Gamma\left(U, \mathcal{D}_{\mathcal{U}^{\dagger} / V}^{\dagger, h}\right)
$$

On est réduit à la trivialité cohomologique du faisceau $\mathcal{O}_{\mathcal{Y}^{\dagger} / V}$ au-dessus du schéma †-adique affine $Y$.

\section{Propriétés de finitude des algèbres d'opérateurs diffé- rentiels $p$-adiques}

Pour tout $h \geq 0$, on a de façon évidente l'inclusion $\mathcal{D}_{\mathcal{X}^{\dagger} / V}^{<\infty, h} \subset \mathcal{D}_{\mathcal{X}^{\dagger} / V}^{\dagger, h}$ et notre but dans les paragraphes qui suivent est de montrer que l'extension précédente est plate et pour cela il nous faut montrer que le faisceau $\mathcal{D}_{\mathcal{X}^{\dagger} / V}^{\dagger, h}$ est noethérien, c'est-à-dire que l'anneau de ses sections globales au-dessus d'un ouvert affine assez petit est noethérien et l'idéal central $\mathfrak{m}$ est contenu son radical pour pouvoir appliquer le critère de platitude local 1.0.3.

\subsection{Radical}

Théorème 4.1.1. Supposant que le schéma $\left(X, \mathcal{O}_{\mathcal{X}_{\dagger} / V}\right)$ est $\dagger$-adique lisse sur $V$, alors si $U$ est un ouvert ayant la propriété (diff) d'algèbre $A^{\dagger}$, pour tout $h \geq 0$ si $P$ est un élément de $D_{A^{\dagger} / V}^{\dagger, h}$ et $\theta$ un élément de l'idéal $\mathfrak{m}$ alors l'opérateur $1-\theta P$ est inversible dans l'anneau $D_{A^{\dagger} / V}^{\dagger, h}$.

Démonstration. L'opérateur $\sum_{m \in \mathbb{N}}(\theta P)^{m}$ est un élément de l'anneau $D_{A^{\wedge} / V}^{\wedge, h}$. La condition sur le degré des réductions modulo les puissances de l'idéal $\mathfrak{m}$ pour l'opérateur $P$ entraîne la condition sur degré des réduction modulo les puissances de l'idéal $\mathfrak{m}$ pour l'opérateur $\sum_{k \in \mathbb{N}}(\theta P)^{k}$. Il reste à montrer que l'opérateur $\sum_{k \in \mathbb{N}}(\theta P)^{k}$ opère sur $A^{\dagger}$. 
Soit $\left(V\left[Y_{1}, \ldots, Y_{m}\right]\right)^{\dagger} \rightarrow A^{\dagger}$ une présentation de l'algèbre $A^{\dagger}$. Pour $\rho>1$ assez près de 1 l'opérateur $P$ opère sur $A_{K, \rho}^{\dagger}$ qui est un $K$-espace de Banach. Si $f$ est un élément de $A^{\dagger}$ il suffit de montrer que

$$
(\theta P)^{k}(f)
$$

tend vers zéro dans l'espace $A_{K, \rho}^{\dagger}$ pour $\rho>1$ assez près de 1 quand $k \rightarrow \infty$. Si $\left(x_{1}, \ldots, x_{n}\right)$ est un système de coordonnées locales au-dessus de $U$, en vertu du théorème 3.1.2 l'opérateur $P$ s'écrit

$$
P\left(a, \Delta, \ldots, \Delta^{p^{h}}\right)=\sum_{\beta^{0}, \ldots, \beta^{h} \in\left(\mathbb{N}_{\leq p-1}^{n}\right)^{h} \times \mathbb{N}^{n}} a_{\beta^{0}, \ldots, \beta^{h}}(\Delta)^{\beta^{0}} \ldots\left(\Delta^{p^{h}}\right)^{\beta^{h}}
$$

où $a_{\beta^{0}, \ldots, \beta^{h}}$ est une suite d'éléments de l'algèbre $A_{\rho}^{\dagger}$ pour $\rho>1$ assez près de 1 et il existe un nombre $\lambda, 0<\lambda<1$ et une constante $C>0$ tels que pour un nombre $\rho>1$ assez près de 1 on a la majoration :

$$
\left\|a_{\beta^{0}, \ldots, \beta^{h}}\right\|_{\rho} \leq C \lambda^{\left|\beta^{0}\right|+\cdots+\left|\beta^{h}\right| p^{h}}
$$

pour tout $\beta^{0}, \ldots, \beta^{h} \in\left(\mathbb{N}^{n}\right)_{\leq p-1}^{h} \times \mathbb{N}^{n}$. D'autre part en vertu de la majoration du théorème 2.6.10 on a les majorations fondamentales

$$
\left\|(\Delta)^{\beta^{0}} \ldots\left(\Delta^{p^{h}}\right)^{\beta^{h}}(f)\right\|_{\rho} \leq C_{\rho}\|f\|_{\rho} \rho^{D(h)\left(\left|\beta^{0}\right|+\cdots+\left|\beta^{h}\right| p^{h}\right)}
$$

pour un entier $D(h) \geq 1$. Ceci montre que pour $\rho>1$ assez près de 1 la norme de Banach de l'opérateur $a_{\beta^{0}, \ldots, \beta^{h}}(\Delta)^{\beta^{0}} \ldots\left(\Delta^{p^{h}}\right)^{\beta^{h}}$ tend vers zéro quand $\left|\beta^{h}\right|$ tend vers l'infini. En particulier on a la majoration

$$
\left\|(\theta P)^{k}(f)\right\|_{\rho} \leq\left\|\left(\theta P_{0}\right)^{k}(f)\right\|_{\rho}
$$

où $P_{0}$ est un opérateur d'ordre fini. Il suffit de montrer que $\left\|\left(\theta P_{0}\right)^{k}(f)\right\|_{\rho}$ tend vers zéro quand $k$ tend vers l'infini pour $\rho>1$ assez près de 1 . En vertu de la majoration du théorème 6.4 de l'article [13] si $f$ se représente par une série $\sum_{\gamma \in \mathbb{N}^{m}} b_{\gamma} y^{\gamma}$ l'élément $(\Delta)^{\beta^{0}} \ldots\left(\Delta^{p^{h}}\right)^{\beta^{h}}(f)$ se représente par une série $\sum_{\gamma \in \mathbb{N}^{m}} b_{\gamma, \beta} y^{\gamma}$ et l'on a la majoration

$$
\max _{\beta}\left|b_{\gamma, \beta}\right| \rho^{|\beta|} \leq\left(\max _{\gamma}\left|b_{\gamma}\right| \rho^{|\gamma|}\right) \rho^{D(h)\left(\sum_{0 \leq j \leq h}\left|\beta^{j}\right| p^{h}\right)}
$$

pour un entier $D(h) \geq 1$. Ceci entraîne que pour un opérateur $P_{0}$ d'ordre fini il existe un entier $D\left(P_{0}\right) \geq 1$ tel que si $f$ se représente par une série $\sum_{\gamma \in \mathbb{N}^{m}} b_{\gamma} y^{\gamma}$ alors $P_{0}(f)$ se représente par une série $\sum_{\gamma \in \mathbb{N}^{m}} b_{P_{0}, \gamma} y^{\gamma}$ et l'on a la majoration

$$
\max _{\gamma}\left|b_{P_{0}, \gamma}\right| \rho^{|\gamma|} \leq\left(\max _{\gamma}\left|b_{\gamma}\right| \rho^{|\gamma|}\right) \rho^{D\left(P_{0}\right)}
$$

pour $\rho>1$ assez près de 1 . 
Ceci entraine que pour tout entier $k \geq 1$ on a la majoration

$$
\left\|\left(\theta P_{0}\right)^{k}(f)\right\|_{\rho} \leq\left(|\theta| \rho^{D\left(P_{0}\right)}\right)^{k}\left(\max _{\gamma}\left|b_{\gamma}\right| \rho^{|\gamma|}\right)
$$

et donc pour $\rho>1$ assez près de 1 la limite de $\left\|\left(\theta P_{0}\right)^{k}(f)\right\|_{\rho}$ quand $k$ tend vers l'infini est nulle.

Corollaire 4.1.2. Supposant que le schéma $\left(X, \mathcal{O}_{\mathcal{X}^{\dagger} / V}\right)$ est †-adique lisse sur $V$, alors si $U$ est ouvert ayant la propriété (diff) d'algèbre $A^{\dagger}$ et si $P$ est un élément de $D_{A^{\dagger} / V}^{\dagger}$ et $\theta$ un élément de l'idéal $\mathfrak{m}$ alors l'opérateur $1-\theta P$ est inversible dans l'anneau $D_{A^{\dagger} / V}^{\dagger}$.

Démonstration. En effet $P$ appartient à $D_{A^{\dagger} / V}^{\dagger, h}$ pour un entier $h \geq 0$ en vertu de la proposition 3.1.6 et le corollaire résulte du théorème 4.1.1.

\subsection{Transposition}

Théorème 4.2.1. Supposant que le schéma $\left(X, \mathcal{O}_{\mathcal{X}^{\dagger} / V}\right)$ est $\dagger$-adique lisse sur $V$, alors si $\left(x_{1}, \ldots, x_{n}\right)$ est un système de coordonnées locales au-dessus d'un ouvert $U$ ayant la propriété (diff) d'algèbre $A^{\dagger}$, alors pour tout $h \geq 0$ l'application

$$
\begin{gathered}
P\left(a, \Delta, \ldots, \Delta^{p^{h}}\right)=\sum_{\beta^{0}, \ldots, \beta^{h} \in\left(\mathbb{N}_{\leq p-1}^{n}\right)^{h} \times \mathbb{N}^{n}} a_{\beta^{0}, \ldots, \beta^{h}}(\Delta)^{\beta^{0}} \ldots\left(\Delta^{p^{h}}\right)^{\beta^{h}} \longmapsto \\
{ }^{t} P\left(a, \Delta, \ldots, \Delta^{p^{h}}\right):=\sum_{\beta^{0}, \ldots, \beta^{h} \in\left(\mathbb{N}_{\leq p-1}^{n}\right)^{h} \times \mathbb{N}^{n}}(-1)^{\left|\beta^{0}\right|+\cdots+\left|\beta^{h}\right| p^{h}}(\Delta)^{\beta^{0}} \ldots\left(\Delta^{p^{h}}\right)^{\beta^{h}} a_{\beta^{0}, \ldots, \beta^{h}}
\end{gathered}
$$

est une anti-involution de l'algèbre $D_{A^{\dagger} / V}^{\dagger, h}$.

Démonstration. Il faut montrer que ${ }^{t} P\left(a, \Delta, \ldots, \Delta^{p^{h}}\right)$ est un opérateur différentiel d'échelon $h$. Il est clair que c'est un opérateur formel et qu'il a la condition sur le degré pour des réduction modulo les puissances de l'idéal $\mathfrak{m}$. Il reste à montrer qu'il opère sur $A^{\dagger}$. Soit $\left(V\left[Y_{1}, \ldots, Y_{m}\right]\right)^{\dagger} \rightarrow A^{\dagger}$ une présentation de l'algèbre $A^{\dagger}$, en vertu du théorème 3.1.2 il existe un nombre $\rho>1$ assez près de 1 tel que les coefficients $a_{\beta^{0}, \ldots, \beta^{h}}$ appartiennent à $A_{\rho}^{\dagger}$ et l'on a les majorations

$$
\| a_{\beta^{0}, \ldots, \beta^{h}}||_{\rho} \leq C_{\rho} \lambda^{\left|\beta^{0}\right|+\cdots+\left|\beta^{h}\right| p^{h}}
$$

pour $\lambda, 0<\lambda<1$ et $C_{\rho}>0$. Soit $f$ un élément de $A_{\rho}^{\dagger}$ pour $\rho>1$, en vertu de la majoration du théorème 2.6.10 on a les majorations

$$
\begin{aligned}
\left\|(\Delta)^{\beta^{0}} \ldots\left(\Delta^{p^{h}}\right)^{\beta^{h}}\left(a_{\beta^{0}, \ldots, \beta^{h}} f\right)\right\|_{\rho} & \leq C_{\rho}^{\prime}\left\|a_{\beta^{0}, \ldots, \beta^{h}} f\right\|_{\rho} \rho^{D(h)\left(\left|\beta^{0}\right|+\cdots+\left|\beta^{h}\right| p^{h}\right)} \\
& \leq C_{\rho}^{\prime \prime} \lambda^{\left|\beta^{0}\right|+\cdots+\left|\beta^{h}\right| p^{h}} \rho^{D(h)\left(\left|\beta^{0}\right|+\cdots+\left|\beta^{h}\right| p^{h}\right)}
\end{aligned}
$$


pour des constantes positives $C_{\rho}^{\prime}, C_{\rho}^{\prime \prime}, D(h)$. Ces majorations montrent que pour $\rho>1$ assez près de 1 , l'élément $(\Delta)^{\beta^{0}} \ldots\left(\Delta^{p^{h}}\right)^{\beta^{h}}\left(a_{\beta^{0}, \ldots, \beta^{h}} f\right)$ tend vers zéro dans $A_{\rho}^{\dagger}$ quand $\left|\beta^{h}\right|$ tend vers l'infini et donc ${ }^{t} P\left(a, \Delta, \ldots, \Delta^{p^{h}}\right)$ opère sur l'algèbre $A^{\dagger}$.

Corollaire 4.2.2. Supposant que le schéma $\left(X, \mathcal{O}_{\mathcal{X}^{\dagger} / V}\right)$ est $V$-lisse et $U$ est un ouvert ayant la propriété (diff) d'algèbre $A^{\dagger}$, alors l'application

$$
P=\sum_{\alpha} a_{\alpha} \Delta^{\alpha} \rightarrow{ }^{t} P:=\sum_{\alpha}(-1)^{\alpha} \Delta^{\alpha} a_{\alpha}
$$

est une anti-involution de l'algèbre des opérateurs différentiels p-adiques $D_{A^{\dagger} / V}^{\dagger}$ qui respecte la filtration par les échelons.

Démonstration. C'est une conséquence du théorème précédent en vertu de la filtration par les échelons.

Remarque 4 .2.3. Le lecteur notera que le théorème 4.1.1 et le théorème 4.2.1 reposent la majoration du théorème 2.6.10 et de ce fait ne sont pas vraiment de nature élémentaire malgré les apparences.

\subsection{Noethérianité}

Proposition 4.3.1. Supposant que le schéma $\left(X, \mathcal{O}_{\mathcal{X}^{\dagger} / V}\right)$ est $\dagger$-adique lisse sur $V$, alors si $U$ est un ouvert ayant la propriété (diff) d'algèbre $A^{\dagger}$ pour tout $h \geq 0$ les anneaux $D_{A^{\dagger} / V}^{<\infty, h}$ et $D_{A^{\wedge} / V}^{\wedge, h}$ sont noethériens.

Démonstration. En effet la filtration par l'ordre des opérateurs différentiels de l'anneau $D_{A^{\dagger} / V}^{<\infty, h}$ est exhaustive et discrète dont le gradué $A^{\dagger}\left[\xi^{0}, \ldots, \xi^{h}\right]$ $:=A^{\dagger}\left[\xi_{1}^{0}, \ldots, \xi_{n}^{h}\right]$ est l'algèbre commutative définie en 3.1.1 qui est type fini sur un anneau noethérien est noethérienne. Il résulte par un argument classique que l'anneau $D_{A^{\dagger} / V}^{<\infty, h}$ est noethérien. L'anneau $D_{A^{\wedge} / V}^{\wedge, h}$ est le complété séparé pour la topologie définie par un idéal central d'un anneau noethérien. D'autre part le $D_{A^{\wedge} / V}^{\wedge, h} / \mathfrak{m}$-module $\mathfrak{m} D_{A^{\wedge} / V}^{\wedge, h} / \mathfrak{m}^{2}$ est de type fini, ceci entraîne que l'anneau $D_{A^{\wedge} / V}^{\wedge, h}$ est lui-même noethérien ([3, Chap. III, $\S 2, \mathrm{n}^{\circ} 10$, Coro. 5]).

L'argument précédent ne s'applique pas à l'anneau $D_{A^{\dagger} / V}^{\dagger, h}$, aussi la noethérianité de cet anneau est beaucoup plus délicate parce que nous ne savons pas la déduire du cas formel.

Pour montrer que l'anneau $D_{A^{\dagger} / V}^{\dagger, h}$ est noethérien nous allons montrer que c'est un quotient d'un anneau qui admet un algorithme de division pour un ouvert affine $U$ assez petit. 


\section{Le théorème de division dans l'anneau $\left(V[Y, Z]_{\mathcal{R}}\right)^{\dagger}$}

\subsection{L'anneau $\left(V\left[Y_{1}, \ldots, Y_{m}\right]\left[Z^{0}, \ldots, Z^{h}\right]_{\mathcal{R}}\right)^{\dagger}$}

Pour $0 \leq j \leq h$ si $\beta^{j}:=\left(\beta_{1}^{j}, \ldots, \beta_{n}^{j}\right)$ est un élément de $\mathbb{N}^{n}$ notons $\left(Z^{j}\right)^{\beta^{j}}$ le monôme $\left(Z_{1}^{j}\right)^{\beta_{1}^{j}} \ldots\left(Z_{n}^{j}\right)^{\beta_{n}^{j}}$ en les indéterminées $Z_{i}^{j}, 1 \leq i \leq n$. Si $\beta:=\left(\beta^{0}, \ldots, \beta^{h}\right)$ est un élément de $\left(\mathbb{N}^{n}\right)^{h+1}$ notons $Z^{\beta}$ le monôme $\left(Z^{0}\right)^{\beta^{0}} \ldots\left(Z^{h}\right)^{\beta^{h}}$.

Définition 5.1.1. Soient trois entiers $m \geq 1, n \geq 1, h \geq 0$ et

$$
\mathcal{R}:=\mathcal{R}_{m, n, h}:=\left\{R_{q, i}^{k} \in V\left[Y_{1}, \ldots, Y_{m}\right], 1 \leq q \leq m, 1 \leq i \leq n, 1 \leq k \leq p^{h}\right\}
$$

un ensemble de polynômes en les indéterminées $Y:=\left(Y_{1}, \ldots, Y_{m}\right)$.

On définit et on note $V[Y, Z]_{\mathcal{R}}$ la $V$-algèbre associative non commutative dans laquelle $V$ est central, engendrée par les indéterminées $Y, Z$, $Y:=\left(Y_{1}, \ldots, Y_{m}\right), Z:=\left(Z_{1}^{0}, \ldots, Z_{n}^{h}\right)$ à coefficients dans $V$ soumises aux relations :

1) $\left[Y_{q}, Y_{q^{\prime}}\right]=0,\left[Z_{i}^{j}, Z_{i^{\prime}}^{j^{\prime}}\right]=0,1 \leq q \leq q^{\prime} \leq m, 1 \leq i \leq i^{\prime} \leq n, 0 \leq j \leq$ $j^{\prime} \leq h$,

2) $\left[Z_{i}^{j}, Y_{q}\right]:=Z_{i}^{j} Y_{q}-Y_{q} Z_{i}^{j}:=\sum_{1 \leq k \leq p^{j}} u_{p^{j}-k} R_{q, i}^{k}\left(Z_{i}^{0}\right)^{a_{p^{j}-k, 0}} \ldots\left(Z_{i}^{j}\right)^{a_{p^{j}-k, j}}$ où $k=\sum_{0 \leq r \leq j} a_{k, r} p^{r}$ est le développement $p$-adique de $k$ et où $u_{k}:=$ $\frac{(p !)^{a} k, 1 \ldots\left(p^{j-1} !\right)^{a} \overline{k, j-1}}{k !}$.

Tout élément de cette algèbre $V[Y, Z]_{\mathcal{R}}$ s'exprime de façon unique comme une somme finie de monômes à coefficients dans $V$ :

$$
\sum_{\alpha, \beta \in \mathbb{N}^{m} \times\left(\mathbb{N}^{n}\right)^{h+1}} a_{\alpha, \beta} Y^{\alpha} Z^{\beta} .
$$

Définition 5.1.2. Soit $Y^{\alpha} Z^{\beta}$ un monôme, on définit :

1) le degré en $Y \operatorname{deg}_{Y}\left(Y^{\alpha} Z^{\beta}\right):=|\alpha|$,

3) le degré en $Z \operatorname{deg}_{Z}\left(Y^{\alpha} Z^{\beta}\right):=|\beta|:=\left|\beta^{0}\right|+\cdots+\left|\beta^{h}\right|$,

4) le degré total $\operatorname{deg}\left(Y^{\alpha} Z^{\beta}\right):=|\alpha|+|\beta|$,

5) l'ordre $\operatorname{ord}\left(Y^{\alpha} Z^{\beta}\right):=\operatorname{ord}\left(Z^{\beta}\right):=\operatorname{ord}(\beta):=\left|\beta^{0}\right|+\cdots+p^{h}\left|\beta^{h}\right|$.

6) On définit la constante $C_{\mathcal{R}}$ comme $\max \left(1, \max _{q, i, k} \operatorname{deg}_{Y} R_{q, i}^{k}\right)$.

Lemme 5.1.3. Soient $j, 0 \leq j \leq h$ un entier et $\alpha, \beta$ un élément de $\mathbb{N}^{m} \times$ $\left(\mathbb{N}^{n}\right)^{j+1}, \beta=\left(\beta^{0}, \ldots, \beta^{j}\right)$. On a alors l'égalité :

$$
\left[Z^{\beta}, Y^{\alpha}\right]=\sum_{0 \leq \beta^{\prime}<\beta} A_{\alpha, \beta-\beta^{\prime}}(Y) Z^{\beta^{\prime}}
$$

où $\beta^{\prime} \in\left(\mathbb{N}^{n}\right)^{j+1}$ et $A_{\alpha, \beta-\beta^{\prime}}(Y)$ est un polynôme en $Y$ dont le degré total est borné $\operatorname{par}|\alpha|+C_{\mathcal{R}}\left(\operatorname{ord} \beta-\operatorname{ord} \beta^{\prime}\right)$. 
Démonstration. Cas 1) $j=0,\left|\beta^{0}\right|=\beta_{i}^{0}=1$. Les relations de la définition 5.1.1 $\left[Z_{i}^{0}, Y_{q}\right]:=R_{q, i}^{0}, 1 \leq q \leq m$ montrent par récurrence sur $|\alpha|$ l'égalité

$$
\left[Z_{i}^{0}, Y^{\alpha}\right]=\sum_{1 \leq q \leq m} \alpha_{q} Y_{1}^{\alpha_{1}} \ldots Y_{q}^{\alpha_{q}-1} \ldots Y_{m}^{\alpha_{m}} R_{q, i}^{0}
$$

et la majoration du lemme est vraie.

Cas 2) $j=0,\left|\beta^{0}\right| \geq 2$. Supposons $\beta_{i}^{0} \geq 1$ et posons

$$
\beta^{0}=\beta^{0^{\prime}}+(0, \ldots, 1, \ldots, 0)
$$

où 1 est placé en $i$-ème position. Il vient

$$
\left[Z^{\beta^{0}}, Y^{\alpha}\right]:=Z^{\beta^{0}} Y^{\alpha}-Y^{\alpha} Z^{\beta^{0}}=\left[Z_{i}^{0}, Y^{\alpha}\right] Z^{\beta^{0^{\prime}}}+Z_{i}^{0}\left[Z^{\beta^{0^{\prime}}}, Y^{\alpha}\right] .
$$

La majoration du lemme a bien lieu pour le terme de droite en vertu du cas 1) et elle aussi lieu pour le terme de gauche par récurrence sur $\left|\beta^{0}\right|$.

Cas 3) Raisonnons par récurrence sur $j \geq 1$. Supposant vraie la formule pour $j-1$.

Soit $|\beta|=\left|\beta^{j}\right|=\beta_{i}^{j}=1$. Si $|\alpha|=1$ la majoration du lemme a lieu en vertu des relations 5.1.1. Supposons que $|\alpha| \geq 2$ et $\alpha_{q} \geq 1$ et posons $\alpha=\alpha^{\prime}+(0, \ldots, 1, \ldots, 0)$ où 1 est placé en $q$-ème position. Il vient

$$
\left[Z_{i}^{j}, Y^{\alpha}\right]=Y^{\alpha^{\prime}}\left[Z_{i}^{j}, Y_{q}\right]+\left[Z_{i}^{j}, Y^{\alpha^{\prime}}\right] Y_{q} .
$$

La majoration du lemme a lieu pour le terme de droite en vertu des relations 5.1.1 et elle a lieu aussi pour le terme de gauche en vertu d'abord de l'hypothèse de récurrence sur $|\alpha|$ puis en vertu de l'hypothèse de récurrence que $j$.

Cas 4) Supposons que $\left|\beta^{j}\right|=\beta_{i}^{j}=1$ et posons $Z^{\beta}:=Z_{i}^{j} Z^{\beta^{\prime}}$ où $\beta^{\prime} \in$ $\left(\mathbb{N}^{n}\right)^{j}$. Il vient

$$
\left[Z^{\beta}, Y^{\alpha}\right]=\left[Z_{i}^{j}, Y^{\alpha}\right] Z^{\beta^{\prime}}+Z_{i}^{j}\left[Z^{\beta^{\prime}}, Y^{\alpha}\right] .
$$

La majoration du lemme a lieu pour le commutateur de gauche en vertu du cas 3) et elle a lieu pour le commutateur de droite d'abord en vertu de l'hypothèse de récurrence sur $j$ puis en vertu du cas 3 ).

Cas 5) Supposons que $\left|\beta^{j}\right| \geq 2$ et $\beta_{i}^{j} \geq 1$. Posons $Z^{\beta}:=Z_{i}^{j} Z^{\beta^{\prime}}$. Il vient

$$
\left[Z^{\beta}, Y^{\alpha}\right]=\left[Z_{i}^{j}, Y^{\alpha}\right] Z^{\beta^{\prime}}+Z_{i}^{j}\left[Z^{\beta^{\prime}}, Y^{\alpha}\right] .
$$

La majoration du lemme a lieu pour le commutateur de gauche en vertu du cas 4) et elle a lieu pour le commutateur de droite d'abord en vertu de l'hypothèse de récurrence sur $\left|\beta^{j}\right|$ puis en vertu du cas 3). 
Remarque 5.1.4. Le lecteur trouvera un calcul d'un commutateur analogue dans la théorie complexe dans l'article [14].

Corollaire 5.1.5. Soit un couple $(\alpha, \beta)$ de $\mathbb{N}^{m} \times\left(\mathbb{N}^{n}\right)^{h+1}$, alors le degré total du commutateur $\left[Z^{\beta}, Y^{\alpha}\right]$ est borné par

$$
p^{h} C_{\mathcal{R}}(|\alpha|+|\beta|) .
$$

Démonstration. C'est une conséquence directe de la majoration du lemme 5.1.3. Nous pouvons considérer le complété m-adique

$$
\left(V[Y, Z]_{\mathcal{R}}\right)^{\wedge}:=\left(V\left[Y_{1}, \ldots, Y_{m}, Z^{0}, \ldots, Z^{h}\right]_{\mathcal{R}}\right)^{\wedge}
$$

de la $V$-algèbre $V[Y, Z]_{\mathcal{R}}$ qui est par construction une $V$-algèbre. Un élément de l'algèbre $\left(V[Y, Z]_{\mathcal{R}}\right)^{\wedge}$ s'écrit de manière unique comme une somme infinie

$$
\sum_{\alpha \in \mathbb{N}^{m}, \beta \in\left(\mathbb{N}^{n}\right)^{h+1}} a_{\alpha, \beta} Y^{\alpha} Z^{\beta}
$$

où les coefficients $a_{\alpha, \beta}$ sont des éléments de $V$ dont la valuation $\mathfrak{m}$-adiques tend vers l'infini avec $|\alpha|+|\beta|$.

L'application symbole total :

$$
P=\sum_{\alpha \in \mathbb{N}^{m}, \beta \in\left(\mathbb{N}^{n}\right)^{h+1}} a_{\alpha \beta} Y^{\alpha} Z^{\beta} \mapsto \sigma_{t o t}(P):=\sum_{\alpha \in \mathbb{N}^{m}, \beta \in\left(\mathbb{N}^{n}\right)^{h+1}} a_{\alpha, \beta} Y^{\alpha} \Xi^{\beta}
$$

est un isomorphisme de $V[Y]$-modules à gauche entre $V[Y, Z]_{\mathcal{R}}$ et l'algèbre commutative

$$
V[Y, \Xi]:=V\left[Y_{1}, \ldots, Y_{m}, \Xi^{0}, \ldots, \Xi^{h}\right]
$$

des polynômes en les indéterminées $Y_{q}, \Xi_{i}^{j}, 1 \leq q \leq m, 1 \leq i \leq n, 0 \leq j \leq h$ à coefficients dans l'anneau $V$.

L'application symbole total se prolonge en un isomorphisme de $(V[Y])^{\wedge}$ modules à gauche entre $(V[Y, Z])^{\wedge}$ et l'algèbre commutative complété

$$
(V[Y, \Xi])^{\wedge}:=\left(V\left[Y_{1}, \ldots, Y_{m}, \Xi^{0}, \ldots, \Xi^{h}\right]\right)^{\wedge} .
$$

Définition 5.1.6. On définit dans le complété $\left(V[Y][Z]_{\mathcal{R}}\right)^{\wedge}$ le $V$-sous-module

$$
\left(V[Y, Z]_{\mathcal{R}}\right)^{\dagger}:=\left(V\left[Y_{1}, \ldots, Y_{m}, Z^{0}, \ldots, Z^{h}\right]_{\mathcal{R}}\right)^{\dagger}
$$

comme l'image, par l' inverse de l'application $\sigma_{\text {tot }}$ symbole total, de la $V$ algèbre

$$
(V[Y, \Xi])^{\dagger}:=\left(V\left[Y_{1}, \ldots, Y_{m}, \Xi^{0}, \ldots, \Xi^{h}\right]\right)^{\dagger}
$$

complété †-adique de l'algèbre commutative $V[Y, \Xi]$. 
Par construction l'application symbole total $\sigma_{\text {tot }}$ est un isomorphisme de $(V[Y])^{\dagger}$-modules à gauche entre $\left(V[Y, Z]_{\mathcal{R}}\right)^{\dagger}$ et $(V[Y, \Xi])^{\dagger}$.

Lemme 5.1.7. Un élément $P$ de $(V[Y, Z])^{\wedge}$ appartient $\grave{a}(V[Y, Z])^{\dagger}$ si et seulement si il s'écrit comme une somme infinie

$$
P(Y, Z)=\sum_{0 \leq l \leq \infty} P_{l}(Y, Z)
$$

où chaque $P_{l}$ est une somme finie de monômes en les variables $Y, Z$ de degré total borné par $C(P)(l+1)$ pour une constante $C(P)>0$ et à coefficients dans $\mathfrak{m}^{l}$.

Démonstration. C'est une conséquence de l'isomorphisme de l'application symbole totale et de la propriété analogue pour les élément de l'algèbre commutative $(V[Y, \Xi])^{\dagger}$.

Lemme 5.1.8. Le $V$-module $\left(V[Y, Z]_{\mathcal{R}}\right)^{\dagger}$ est un sous-anneau de l'anneau $\left(V[Y, Z]_{\mathcal{R}}\right)^{\wedge}$.

Démonstration. Il faut montrer que le $V$-module $(V[Y, Z])^{\dagger}$ est stable par produit. Il nous faut montrer en vertu du lemme précédent que le produit de la forme

$$
P_{l_{1}}(Y, Z) Q_{l_{2}}(Y, Z)
$$

est une somme de monômes de degré borné par $C(P, Q)\left(l_{1}+l_{2}+1\right)$ pour une constante $C(P, Q)>0$. Mais c'est une conséquence de la majoration du degré total d'un commutateur du corollaire 5.1.5.

\subsection{Le Théorème de division dans l'anneau $\left(V[Y, Z]_{\mathcal{R}}\right)^{\dagger}$}

\subsubsection{Division dans l'anneau $\left(V[Y, Z]_{\mathcal{R}}\right)^{\wedge}$}

Soit $g=\sum_{\alpha \in \mathbb{N}^{m}, \beta \in\left(\mathbb{N}^{n}\right)^{h+1}} a_{\alpha, \beta} Y^{\alpha} Z^{\beta}$ un élément de l'anneau $\left(V[Y, Z]_{\mathcal{R}}\right)^{\wedge}$.

Définition 5.2.1. 1) On définit la valuation $\mathfrak{m}$-adique de g par

$$
v(g):=v_{\mathfrak{m}}(g):=\inf _{\alpha, \beta} v\left(a_{\alpha, \beta}\right) .
$$

2) On définit la partie initiale In(g) par:

$$
\operatorname{In}(g):=\sum_{\alpha, \beta, v\left(a_{\alpha, \beta}\right)=v(g)} a_{\alpha, \beta} Y^{\alpha} Z^{\beta} .
$$

3) On définit le nuage de Newton $\mathcal{N}(g)$ par:

$$
\mathcal{N}(g):=\left\{\alpha, \beta \in \mathbb{N}^{m} \times\left(\mathbb{N}^{n}\right)^{h+1}, a_{\alpha, \beta} \neq 0\right\}
$$


Définition 5.2.2. Soient des nombres réels strictement positifs

$$
a=\left(a_{1}, \ldots, a_{m}\right), b^{0}=\left(b_{1}^{0}, \ldots, b_{n}^{0}\right), \ldots, b^{h}=\left(b_{1}^{h}, \ldots, b_{n}^{h}\right),
$$

nous dirons qu'une forme linéaire $L:=L_{a, b}: \mathbb{N}^{m} \times\left(\mathbb{N}^{n}\right)^{h+1} \rightarrow \mathbb{R}^{+}$est adaptée à $\mathcal{R}$ si :

$\left(\mathrm{i}_{h}\right)$ elle est de la forme

$$
\begin{aligned}
& \qquad(\alpha, \beta):=\langle a, \alpha\rangle+\left\langle b_{1}^{0}, \beta^{0}\right\rangle+p\left\langle b^{1}, \beta^{1}\right\rangle+\ldots p^{h}\left\langle b^{h}, \beta^{h}\right\rangle, \\
& \text { pour }\langle a, \alpha\rangle:=a_{1} \alpha_{1}+\cdots+a_{m} \alpha_{m},\left\langle b^{0}, \beta^{0}\right\rangle:=b_{1}^{1} \beta_{1}^{1}+\ldots b_{n}^{1} \beta_{n}^{1} \ldots,\left\langle b^{h}, \beta^{h}\right\rangle \\
& :=b_{1}^{h} \beta_{1}^{1}+\ldots b_{n}^{h} \beta_{n}^{h},
\end{aligned}
$$

(ii) $:=(\text { ii })_{a, b}$ les coefficients $a_{1}, \ldots, a_{m}, b_{1}^{0}, \ldots, b_{n}^{h}$ sont linéairement indépendants sur $\mathbb{Z}$,

$\left(\right.$ iii $\left._{\mathcal{R}}\right)$ on a les majorations strictes $b_{i}^{j}>p^{j} C_{\mathcal{R}}\left(a_{1}+\cdots+a_{m}\right)$ pour $1 \leq i \leq n$, $0 \leq j \leq h$.

Définition 5.2.3. 1) Étant donné une forme linéaire $L$ adaptée à $\mathcal{R}$, on définit l'ordre $<_{L}$ sur l'ensemble $\mathbb{N}^{m} \times\left(\mathbb{N}^{n}\right)^{h+1}$ par

$$
\left(\alpha^{\prime}, \beta^{\prime}\right)<_{L}(\alpha, \beta) \text {, si } L\left(\alpha^{\prime}, \beta^{\prime}\right)<L(\alpha, \beta) \text {. }
$$

La condition (ii) assure que c'est un ordre total compatible avec l'action de $\mathbb{N}^{m} \times\left(\mathbb{N}^{n}\right)^{h+1}$ sur lui-même par translation.

2) Étant donné un élément $g$ de l'anneau $\left(V[Y, Z]_{\mathcal{R}}\right)^{\wedge}$ on définit l'exposant $\exp (\operatorname{In}(g)):=\exp _{L}(\operatorname{In}(g))$ comme le plus grand élément $(\alpha, \beta)$ pour l'ordre $<_{L}$ des monômes qui interviennent dans In $(g)$. On définit l'exposant $\exp (g)$ comme $\exp (\operatorname{In}(g))$.

3) Si $\left(\alpha^{0}, \beta(0)\right)$ est l'exposant de $g$ on définit son symbole principal $\sigma(g)$ pour la forme $L$ par

$$
\sigma(g):=\sigma_{L}(g):=\sigma_{L}(\operatorname{In}(g)):=a_{\alpha^{0}, \beta(0)} Y^{\alpha^{0}} Z^{\beta(0)} .
$$

Lemme 5.2.4. Soient $g_{1}, g_{2}$ deux éléments de l'anneau $\left(V[Y, Z]_{\mathcal{R}}\right)^{\wedge}$, on a alors l'égalité $\exp \left(g_{1} g_{2}\right)=\exp \left(g_{1}\right)+\exp \left(g_{2}\right)$ et si $\exp \left(g_{2}\right)<_{L} \exp \left(g_{1}\right)$ alors $\exp \left(g_{1}+g_{2}\right)=\exp \left(g_{1}\right)$.

Démonstration. La second égalité est évidente. Soient $Y^{\alpha}, Z^{\beta}$ des monômes, alors on a l'inégalité essentielle :

$$
\exp \left(\left[Z^{\beta}, Y^{\alpha}\right]<_{L}(\alpha, \beta)\right.
$$

qui est conséquence de l'expression 5.1.3 d'un commutateur et de la condition $\left(\right.$ iii $\left._{\mathcal{R}}\right)$ d'une forme linéaire adaptée à $\mathcal{R}$. La première égalité du lemme est conséquence de l'inégalité précédente. 
Théorème 5.2.5. Soient $F_{1}, \ldots, F_{d}$ des éléments non nuls et de valuation $\mathfrak{m}$-adique nulle de l'anneau $\left(V[Y, Z]_{\mathcal{R}}\right)^{\wedge}$. Notons

$$
\begin{aligned}
& \Delta_{1}:=\mathbb{N}^{m} \times\left(\mathbb{N}^{n}\right)^{h+1}+\exp \left(F_{1}\right) \\
& \left.\Delta_{i}:=\mathbb{N}^{m} \times\left(\mathbb{N}^{n}\right)^{h+1}+\exp \left(F_{i}\right)\right) \backslash \bigcup_{j=1}^{i-1} \Delta_{j}, \quad i=2, \ldots, d \\
& \bar{\Delta}:=\mathbb{N}^{m} \times\left(\mathbb{N}^{n}\right)^{h+1} \backslash \bigcup_{i=1}^{d} \Delta_{i}=\mathbb{N}^{m} \times\left(\mathbb{N}^{n}\right)^{h+1} \backslash \bigcup_{i=1}^{d}\left(\mathbb{N}^{m} \times\left(\mathbb{N}^{n}\right)^{h+1}+\exp \left(F_{i}\right)\right) .
\end{aligned}
$$

Alors, pour chaque élément $g$ de l'anneau $\left(V[Y, Z]_{\mathcal{R}}\right)^{\wedge}$ ils existent des éléments $q_{1}, \ldots, q_{d}, r$ de l'anneau $\left(V[Y, Z]_{\mathcal{R}}\right)^{\wedge}$ uniques tels que

$$
\begin{aligned}
& \text { 1. } g=\sum_{i=1}^{d} q_{i} F_{i}+r, \\
& \text { 2. } \mathcal{N}\left(q_{i}\right)+\exp \left(F_{i}\right) \subseteq \Delta_{i} \text { pour tout } i=1, \ldots, d \text {, } \\
& \text { 3. } \mathcal{N}(r) \subseteq \bar{\Delta} \text {. }
\end{aligned}
$$

Pour démontrer le théorème nous allons procéder par réduction modulo $\mathfrak{m}$ ([17], [13]). Soit $k[Y, Z]_{\overline{\mathcal{R}}}$ l'anneau réduction modulo $\mathfrak{m}$ de l'anneau $V[Y, Z]_{\mathcal{R}}$. On peut considérer la réduction $\overline{\mathcal{R}}$ de $\mathcal{R}$ et la forme $L$ est adaptée à $\overline{\mathcal{R}}$. On définit de façon évidente le nuage de Newton $\mathcal{N}(\bar{g})$ et l'exposant $\exp (\bar{g})$. L'application exposant de la réduction a les propriétés du lemme 5.2.4.

Proposition 5.2.6. Soient $\bar{F}_{1}, \ldots, \bar{F}_{d}$ des éléments non nuls de l'anneau $k[Y, Z]_{\overline{\mathcal{R}}}$. Alors, pour chaque élément $\bar{g}$ de l'anneau $k[Y, Z]_{\overline{\mathcal{R}}}$ ils existent des éléments $\bar{q}_{1}, \ldots, \bar{q}_{d}, \bar{r}$ de l'anneau $k[Y, Z]_{\overline{\mathcal{R}}}$ uniques tels que

$$
\text { 1. } \bar{g}=\sum_{i=1}^{d} \bar{q}_{i} \bar{F}_{i}+\bar{r},
$$

2. $\mathcal{N}\left(\bar{q}_{i}\right)+\exp \left(\bar{F}_{i}\right) \subseteq \Delta_{i}$ pour tout $i=1, \ldots, d$,

3. $\mathcal{N}(\bar{r}) \subseteq \bar{\Delta}$,

où les ensembles $\Delta_{i}, \bar{\Delta}$ sont définis à partir des exposants $\exp \left(\bar{F}_{i}\right)$.

Démonstration. L'unicité résulte des propriétés des exposants du lemme 5.2.4. Soit $\bar{g}$ un élément non nul de l'anneau $k[Y, Z]_{\overline{\mathcal{R}}}$ quitte à enlever les monômes de $\bar{g}$ qui appartiennent à $\bar{\Delta}$ on peut supposer que l'exposant $\exp (\bar{g})$ appartient à l'ensemble $\bigcup_{i=1}^{d} \Delta_{i}$. Soit $i_{0}$ le plus petit entier $1 \leq i_{0} \leq d$ tel que $\exp (\bar{g}) \in \mathbb{N}^{m} \times\left(\mathbb{N}^{n}\right)^{h+1}+\bar{F}_{i_{0}}$, on a alors l'égalité

$$
\exp (\bar{g})=\left(\alpha^{0}, \beta(0)\right)+\exp \left(\bar{F}_{i_{0}}\right)
$$

pour un élément $\left(\alpha^{0}, \beta(0)\right)$ de l'ensemble $\mathbb{N}^{m} \times\left(\mathbb{N}^{n}\right)^{h+1}$. 
Si $\sigma\left(\bar{F}_{i_{0}}\right)=w_{i_{0}} Y^{\alpha^{i_{0}}} Z^{\beta\left(i_{0}\right)}$ pour un élément $w_{i_{0}}$ du corps résiduel $k$ et l'on a l'égalité :

$$
\sigma(\bar{g})=w Y^{\alpha^{0}} Z^{\beta(0)} \sigma\left(\bar{F}_{i_{0}}\right)-w Y^{\alpha^{0}}\left[Z^{\beta(0)}, Y^{\alpha^{i_{0}}}\right] Z^{\beta\left(i_{0}\right)}
$$

pour un élément $w$ du corps résiduel $k$. Le point est qu'en vertu de la condition $\left(\mathrm{iii}_{\mathcal{R}}\right.$ ) l'exposant du commutateur est plus petit que l'exposant de $\bar{g}$ pour l'ordre $<_{L}$. L'exposant de l'élément $\bar{g}-w Y^{\alpha^{0}} Z^{\beta(0)} \sigma\left(\bar{F}_{i_{0}}\right)$ est petit que l'exposant de $\bar{g}$ pour l'ordre $<_{L}$ et la proposition 5.2.6 résulte par récurrence descendante sur les valeurs discrètes de $L$.

Démonstration du théorème 5.2.5. Soit $g$ un élément de l'anneau $\left(V[Y, Z]_{\mathcal{R}}\right)^{\wedge}$, en choisissant une uniformisante de $V$ on peut supposer que $v(g)$ est nulle. On considère la réduction $\bar{g}$ modulo $\mathfrak{m}$ de $g$ et on divise $\bar{g}$ par la proposition précédente. On obtient

$$
\bar{g}=\sum_{i=1}^{d} \bar{q}_{i} \bar{F}_{i}+\bar{r}
$$

et on remonte les éléments $\bar{q}_{1}, \ldots, \bar{q}_{d}, r$ en respectant les nuages de Newton en des éléments $q_{10}, \ldots q_{0 d}, r_{0}$ de l'anneau $\left(V[Y, Z]_{\mathcal{R}}\right)^{\wedge}$. La valuation m-adique de $g_{1}:=g-\left(\sum_{i=1}^{d} q_{i 0} F_{i}+r_{0}\right)$ est strictement positive. On construit par récurrence sur la valuation $\mathfrak{m}$-adique des éléments $g_{l}, q_{1 l}, \ldots, q_{d l}, r_{l}$ de valuation $\mathfrak{m}$-adique $\geq l$ pour $l \geq 1$. Les éléments $q_{1}:=\sum_{l>0} q_{1 l}, \ldots, q_{d}:=$ $\sum_{l \geq 0} q_{d l}, r:=\sum_{l \geq 0} r_{l}$ ont les propriétés du théorème 5.2.5.

\subsubsection{Division dans l'anneau $\left(V[Y, Z]_{\mathcal{R}}\right)^{\dagger}$}

Soient un ensemble fini de polynômes $\mathcal{R}$ et $L$ une forme linéaire adaptée à $\mathcal{R}$ comme dans le paragraphe précédent.

Théorème 5.2.7. Soient $F_{1}, \ldots, F_{d}$ des éléments non nuls et de valuation $\mathfrak{m}$-adique nulle de l'anneau $\left(V[Y, Z]_{\mathcal{R}}\right)^{\dagger}$. Alors, pour chaque élément $g$ de l'anneau $\left(V[Y, Z]_{\mathcal{R}}\right)^{\dagger}$ les quotients $q_{1}, \ldots, q_{d}$ et le reste $r$ du théorème 5.2.5 sont des éléments de l'anneau $\left(V[Y, Z]_{\mathcal{R}}\right)^{\dagger}$.

Pour passer du théorème 5.2.5 au théorème 5.2.7, nous utilisons la continuité de la division [14] et la méthode maintenant bien connue de la perturbation par commutateur des homéomorphismes entre espaces de Banach ([8], [9], [13]). Nous passons donc au langage des valeurs absolues des corps valués complets. On note $|a|:=|a|_{p}:=p^{-v_{\mathfrak{m}}(a)}$ la valeur absolue $p$-adique d'un élément $a$ de $K$. 
Notons pour simplifier, comme dans l'article [13], $\mathbf{S}:=\left(V[Y, Z]_{\mathcal{R}}\right)^{\wedge} \otimes_{V} K$ et $\mathbf{R}:=\left(V[Y, Z]_{\mathcal{R}}\right)^{\dagger} \otimes_{V} K$ et pour chaque réel $s>1$

$$
\mathbf{R}_{s}=\left\{\sum_{\alpha \in \mathbb{N}^{m}, \beta \in\left(\mathbb{N}^{n}\right)^{h+1}} a_{\alpha, \beta} Y^{\alpha} Z^{\beta}\left|\lim _{|\alpha|+|\beta| \rightarrow+\infty}\right| a_{\alpha, \beta} \mid s^{L(\alpha, \beta)}=0\right\} .
$$

On a donc $\mathbf{R}=\cup_{s>1} \mathbf{R}_{s}$. Notons pour $g \in \mathbf{R}_{s}$,

$$
\||| g||_{s}:=\max _{\alpha, \beta}\left|a_{\alpha, \beta}\right| s^{L(\alpha, \beta)} .
$$

En vertu de la condition $\left(\mathrm{iii}_{\mathcal{R}}\right.$ ) de la définition 5.2 .2 , pour tout $s>1, \mathbf{R}_{s}$ muni de la norme ||$|-\||_{s}$ est une $K$-algèbre de Banach non commutative. On a le lemme suivant ([13, Lem. 2.3.3]) :

Lemme 5.2.8. Si $g \in \mathbf{R}$, il existe un $s_{0}>1$ tel que pour tout $\left.\left.s \in\right] 1, s_{0}\right]$ on a la majoration

$$
\left|\left\|g-\sigma(g)\left|\left\|_{s}<\nu(s)\left|\|\sigma(g) \mid\|_{s}\right.\right.\right.\right.\right.
$$

où $\nu(s)<1$.

Démonstration du théorème 5.2.7. Nous reprenons la démonstration du théorème 2.3.4 de l'article [13]. Notons

$$
\begin{gathered}
\Delta(F)=\{r \in \mathbf{S} \mid \mathcal{N}(r) \subseteq \bar{\Delta}\}, \\
\nabla(F)=\left\{q \in \mathbf{S}^{d} \mid \mathcal{N}\left(q_{i}\right)+\exp \left(F_{i}\right) \subseteq \Delta_{i}, \forall i=1, \ldots, d\right\},
\end{gathered}
$$

et pour chaque $s>1$,

$$
\Delta_{s}(F)=\Delta(F) \cap \mathbf{R}_{s}, \quad \nabla_{s}(F)=\nabla(F) \cap \mathbf{R}_{s}^{d} .
$$

Ce sont des sous-espaces fermés et donc des sous-espaces de Banach des espaces de $\mathbf{R}_{s}$ et $\mathbf{R}_{s}^{d}$ respectivement.

Notons $u: \nabla(F) \oplus \Delta(F) \rightarrow S$ l'application $K$-linéaire induite par le théorème 5.2 .5 définie par

$$
u(q, r)=\sum_{i=1}^{d} q_{i} F_{i}+r
$$

et $w: \nabla(F) \oplus \Delta(F) \rightarrow \mathbf{S}$ l'application $K$-linéaire définie par

$$
w(q, r)=\sum_{i=1}^{d} q_{i}\left(F_{i}-\sigma\left(F_{i}\right)\right)
$$

En vertu du théorème 5.2.5, l'application $u$ est bijective. 
Notons pour chaque $s>1$ suffisamment proche à 1 (tel que $F_{i} \in \mathbf{R}_{s}$ pour tout $i=1, \ldots, d) u_{s}: \nabla_{s}(F) \oplus \Delta_{s}(F) \rightarrow \mathbf{R}_{s}$ et $w_{s}: \nabla_{s}(F) \oplus \Delta_{s}(F) \rightarrow \mathbf{R}_{s}$ les applications linéaires continues induites par $u$ et $w$ respectivement.

L'application $v_{s}:=u_{s}-w_{s}$ se décompose en une somme $v_{s 1}+v_{s 2}$ à cause de la non-commutativité. En effet si $Y^{\alpha^{0}} Z^{\beta(0)}$ est un monôme on a l'égalité pour tout $P$

$$
P Y^{\alpha^{0}} Z^{\beta(0)}:=\left(\sum_{\alpha, \beta} a_{\alpha, \beta} Y^{\alpha} Z^{\beta}\right) Y^{\alpha^{0}} Z^{\beta(0)}=t^{1}(P)+t^{2}(P)
$$

où

$$
t^{1}(P):=\sum_{\alpha, \beta} a_{\alpha, \beta} Y^{\alpha+\alpha^{0}} Z^{\beta+\beta(0)}
$$

et

$$
t^{2}(P):=-\sum_{\alpha, \beta} a_{\alpha, \beta} Y^{\alpha}\left[Z^{\beta}, Y^{\alpha^{0}}\right] Z^{\beta(0)}
$$

Posons

$$
v_{s 1}(q, r):=t_{1}^{1}\left(q_{1}\right)+\ldots t_{d}^{1}\left(q_{d}\right)+r, v_{s 2}(q):=t_{1}^{2}\left(q_{1}\right)+\ldots t_{d}^{2}\left(q_{d}\right)
$$

où les applications $t_{1}^{1}, \ldots, t_{d}^{1}, t_{1}^{2} \ldots, t_{d}^{2}$ sont définies par $\sigma\left(F_{1}\right), \ldots, \sigma\left(F_{d}\right)$ par le processus du commutateur précédent.

La division du théorème 5.2.5 par des monômes montre que l'application $v_{s 1}$ est bijective et l'on a l'égalité des normes

$$
\left|\left\|v_{s 1}(q, r) \mid\right\|_{s}=\max _{i}\left\{| | | q _ { i } | \left\|_ { s } \cdot \left|\left\|\sigma ( F _ { i } ) \left|\left\|_{s},\left|\|r \mid\|_{s}\right\},\right.\right.\right.\right.\right.\right.\right.
$$

pour $s>1$ suffisamment proche à 1 .

Considérons donc sur l'espace de Banach $\nabla_{s}(F) \oplus \Delta_{s}(F)$ la norme équivalente

$$
\text { I|| }(q, r) \mid \|_{s}^{\prime}=\max \left\{| | | q _ { i } | \left\|_ { s } \cdot \left|\left\|\sigma ( F _ { i } ) \left|\left\|_{s},\left|\|\underline{r} \mid\|_{s}\right\},\right.\right.\right.\right.\right.\right.
$$

dans laquelle l'isomorphisme $v_{s 1}$ a une norme égale à 1 .

On a alors $u_{s}=v_{s 1}+v_{s 2}+w_{s}$.

Or, d'après le lemme 5.2.8, il existe un $s_{0}>1$ tel que pour tout $\left.\left.s \in\right] 1, s_{0}\right]$ et pour tout $i=1, \ldots, d$ on a $\left|\left\|F_{i}-\sigma\left(F_{i}\right)\left|\left\|_{s}<\nu(s)\left|\left\|\sigma\left(F_{i}\right) \mid\right\|_{s}\right.\right.\right.\right.\right.$, avec $\nu(s)<1$. Ceci entraîne que la norme de l'application $w_{s}$ est plus petite ou égale à $\nu(s)<1$.

En vertu de la condition de la condition $\left(i i_{\mathcal{R}}\right)$ de la définition 5.2 .2 on a les majorations

$$
\begin{aligned}
\left\|\left|t_{1}^{2}\left(q_{1}\right)\right|\right\|_{s} & \leq s^{-\left(\min b_{i}^{j}-a_{q}\right)}\left|\left\|q_{1} \sigma\left(F_{d}\right)\left|\left\|_{s}, \ldots,\right\|\right|\right\| t_{d}^{2}\left(q_{d}\right)\|\|_{s}\right. \\
& \leq s^{-\left(\min b_{i}^{j}-a_{q}\right)}\|\| q_{d} \sigma\left(F_{d}\right)\|\|_{s}
\end{aligned}
$$

où les nombres réels positifs $a_{q}, 1 \leq q \leq m, b_{i}^{j}, 1 \leq i \leq n, 0 \leq j \leq h$ sont ceux de la définition 5.2.2 d'une forme adaptée $L:=L_{a, b}$ à $\mathcal{R}$. 
Ces majorations montrent que la norme de l'application $v_{s 2}$ est bornée par $s^{-\left(\min b_{i}^{j}-a_{q}\right)}$ donc strictement bornée par 1 en vertu de la condition $\left(\right.$ iii $\left._{\mathcal{R}}\right)$. L'application $u_{s}$ est un isomorphisme topologique isométrique. D'où le théorème 5.2.7.

Remarque 5.2.9. Sur un corps $K$ ultra-métrique complet quelconque, on peut de façon évidente considérer l'anneau $K[Y, Z]_{\mathcal{R}}$ défini à partir d'un ensemble $\mathcal{R}$ de polynômes à coefficients dans l'anneaux des entiers $\mathcal{O}_{K}$ de $K$, son complété $\left(K[Y, Z]_{\mathcal{R}}\right)^{\wedge}$ et le sous-anneaux $\left(K[Y, Z]_{\mathcal{R}}\right)^{\dagger}$. On a un algorithme de division dans l'anneau $\left(K[Y, Z]_{\mathcal{R}}\right)^{\wedge}$ tout à fait parallèle à l'algorithme du théorème 5.2.5 en réduisant non pas modulo l'idéal maximum de l'anneau des entiers mais en considérant l'anneau $k_{r}:=\left\{\mathcal{O}_{K} /(a \in K,|a| \leq r)\right\}$ pour un $r, 0<r<1$ convenable et la réduction $\mathcal{O}_{K}\langle x\rangle \rightarrow k_{r}[x]$ comme dans la division dans le cas commutatif de l'article ([13, Thm. 2.3.2]). Le même raisonnement montre que la division se restreint à l'anneaux $\left(K[Y, Z]_{\mathcal{R}}\right)^{\dagger}$. Ces anneaux sont alors tous noethériens (cf. [17, remark 5.4]), ce qui produit beaucoup d'anneaux noethériens. Le lecteur notera que s'il n'y a pas de variables $Z$ cette division et sa démonstration se réduisent exactement au théorème de division dans le cas commutatif ([13, Thm. 2.3.2]).

Il nous faut généraliser légèrement le théorème 5.2.7 en abandonnant la restriction sur les valuations $\mathfrak{m}$-adiques des diviseurs pour pouvoir considérer tous les idéaux de l'anneau $\left(V[Y, Z]_{\mathcal{R}}\right)^{\dagger}$. Considérons l'ensemble $\mathbb{N} \times$ $\mathbb{N}^{m} \times\left(\mathbb{N}^{n}\right)^{h+1}$ des éléments $(\mu, \alpha, \beta)$. Définissons la relation d'ordre par $\left(\mu^{\prime}, \alpha^{\prime}, \beta^{\prime}\right)<_{L}(\mu, \alpha, \beta)$ si $\mu^{\prime}>\mu$ ou si $\mu^{\prime}=\mu$ et $L\left(\alpha^{\prime}, \beta^{\prime}\right)<L(\alpha, \beta)$ pour une forme linéaire $L$ adaptée à $\mathcal{R}$. C'est une relation d'ordre total. Nous définissons l'exposant précisé $\widetilde{\exp }(P):=\widetilde{\exp }_{L}(P)$ d'un élément $P$ de l'anneau $\left(V[Y, Z]_{\mathcal{R}}\right)^{\wedge}$ comme $\left(v(P), \exp _{L}(P)\right)$. L'application exposant précisé a les propriétés du lemme 5.2.4.

Soient $1 \leq d_{1} \leq d_{2} \leq \cdots \leq d_{l}$ des entiers naturels et pour chaque entier $d_{l^{\prime}}, 1 \leq l^{\prime} \leq l$ des éléments $F_{1}^{l^{\prime}}, \ldots, F_{d_{l^{\prime}}}^{l^{\prime}}$ de l'anneau $\left(V[Y, Z]_{\mathcal{R}}\right)^{\dagger}$ ayant même valuation $\mathfrak{m}$-adique égale à $\mu_{l^{\prime}}$ tels que $0 \leq \mu_{1}<\mu_{2}<\cdots<\mu_{l}$.

Théorème 5.2.10. Avec les notations précédentes, pour chaque élément $g$ de l'anneau $\left(V[Y, Z]_{\mathcal{R}}\right)^{\dagger}$ ils existent des éléments $q_{1}^{1}, \ldots, q_{d_{l}}^{l}, r$ de l'anneau $\left(V[Y, Z]_{\mathcal{R}}\right)^{\dagger}$ uniques tels que

$$
\begin{aligned}
& \text { 1. } g=\sum_{i=1}^{d_{1}} q_{i}^{d_{1}} F_{i}^{1}+\cdots+\sum_{i=1}^{d_{l}} q_{i}^{l} F_{i}^{l}+r \\
& \text { 2. } \mathcal{N}\left(q_{i}^{l^{\prime}}\right)+\widetilde{\exp }\left(F_{i}^{l^{\prime}}\right) \subseteq \Delta_{i}^{l^{\prime}} \text { pour tout } i=1, \ldots, l^{\prime}, 1 \leq l^{\prime} \leq l, \\
& \text { 3. } \mathcal{N}(r) \subseteq \bar{\Delta}
\end{aligned}
$$

où les ensembles $\Delta_{i}^{d_{j}}, \bar{\Delta}$ sont définit comme dans le théorème 5.2.5. 
Démonstration. Soit $v(g)$ la valuation de $g$, si $v(g)<\mu_{1}$ alors $r:=g$. Si $v(g) \geq \mu_{1}$ on divise $g$ par $F_{1}^{1}, \ldots, F_{d_{1}}^{1}$ à l'aide du théorème 5.2.7. Si $r_{1}$ est le reste de cette division, on divise par le processus précédent $r_{1}$ par $F_{1}^{2}, \ldots, F_{2}^{d_{2}}$. On obtient par division successive des restes le quotient et le reste de la division du théorème 5.2.10.

Remarque 5.2.11. On a considéré que la division par un nombre fini d'éléments de $\left(V[Y, Z]_{\mathcal{R}}\right)^{\dagger}$ pour simplifier. Mais on a une division par un nombre fini de vecteurs de $\left(\left(V[Y, Z]_{\mathcal{R}}\right)^{\dagger}\right)^{m}$ tout à fait parallèle. Nous avons rencontré des situations où la division par les vecteurs est nécessaire.

Corollaire 5.2.12. L'anneau $\left(V[Y, Z]_{\mathcal{R}}\right)^{\dagger}$ est noethérien à gauche

Démonstration. Soit $J$ un idéal à gauche de l'anneau $\left(V[Y, Z]_{\mathcal{R}}\right)^{\dagger}$, alors l'ensemble des exposants précisés $\widetilde{E}(J)$ des éléments de $J$ est stable par translation par les éléments de l'ensemble $\mathbb{N} \times \mathbb{N}^{m} \times\left(\mathbb{N}^{n}\right)^{h+1}$. Il résulte du lemme de Dickson que $\widetilde{E}(J)$ est de type fini : il existe un nombre fini d'éléments $F_{1}, \ldots, F_{d}$ de l'idéal $J$ tels que $\widetilde{E}(J)=\cup_{i=1, d} \widetilde{\exp }\left(F_{1}\right)+\mathbb{N} \times$ $\mathbb{N}^{m} \times\left(\mathbb{N}^{n}\right)^{h+1}$. On appelle base de division de l'idéal $J$ un tel système de générateurs. En vertu du théorème 5.2.10 une base de division de l'idéal $J$ est un système de générateurs de $J$.

Nous aurons besoin au paragraphe suivant d'une généralisation des corollaires précédents. Soit un entier $h \geq 0$ notons $\left(V[Y, Z]_{\mathcal{R}^{h}}\right)^{\dagger}$ pour $\left(V[Y, Z]_{\mathcal{R}}\right)^{\dagger}$ de sorte que l'on peut considérer l'extension $\left(V[Y, Z]_{\mathcal{R}^{h}}\right)^{\dagger} \rightarrow\left(V[Y, Z]_{\mathcal{R}^{h+1}}\right)^{\dagger}$. L'anneau $\left(V[Y, Z]_{\mathcal{R}^{h+1}}\right)^{\dagger}$ contient comme sous-anneaux les anneaux

$$
\left(V[Y, Z]_{\mathcal{R}^{h}}\right)^{\dagger}\left[Z_{1}^{h+1}, \ldots, Z_{i}^{h+1}\right]
$$

des éléments d'ordre fini en $Z_{1}^{h+1}, \ldots, Z_{i}^{h+1}$ pour $1 \leq i \leq n$ qui sont des extensions intermédiaires de l'anneau $\left(V[Y, Z]_{\mathcal{R}^{h}}\right)^{\dagger}$.

Corollaire 5.2.13. Les anneaux $\left(V[Y, Z]_{\mathcal{R}^{h}}\right)^{\dagger}\left[Z_{1}^{h+1}, \ldots, Z_{i}^{h+1}\right]$ sont noethériens pour tout $i, 1 \leq i \leq n$.

Démonstration. Considérons d'abord l'anneau $\left(V[Y, Z]_{\mathcal{R}^{h}}\right)^{\dagger}\left[Z_{1}^{h+1}\right]$ qui est un anneau filtré par l'ordre en $Z_{1}^{h+1}$ qui attribue l'ordre $p^{h+1}$ à $Z_{1}^{h+1}$. En vertu des relations de la définition 5.1.1 le commutateur des deux éléments de l'anneau $\left(V[Y, Z]_{R^{h}}\right)^{\dagger}\left[Z_{1}^{h+1}\right]$ est d'ordre strictement plus petit que la somme des ordres de ces deux éléments. L'anneau gradué associé pour cette filtration est l'anneau des polynômes $\left(V[Y, Z]_{R^{h}}\right)^{\dagger}\left[\Xi_{1}^{h+1}\right]$ en la variable $\Xi_{1}^{h+1}$ à coefficients dans l'anneau non-commutatif $\left(V[Y, Z]_{R^{h}}\right)^{\dagger}$ mais où l'indéterminée $\Xi_{1}^{h+1}$ est centrale. 
En vertu de la démonstration classique du théorème de la base finie de Hilbert dans le cas commutatif, l'anneau $\left(V[Y, Z]_{\mathcal{R}^{h}}\right)^{\dagger}\left[\Xi_{1}^{h+1}\right]$ est noethérien. Par suite l'anneau $\left(V[Y, Z]_{R^{h}}\right)^{\dagger}\left[Z_{1}^{h+1}\right]$ muni d'une filtration croissante discrète dont le gradué est noethérien est lui-même noethérien. On passe par le raisonnement précédent au cas des anneaux $\left(V[Y, Z]_{\mathcal{R}^{h}}\right)^{\dagger}\left[Z_{1}^{h+1}, \ldots, Z_{i}^{h+1}\right]$ par récurrence sur $i$. D'où le corollaire.

Remarque 5.2.14. La noethérianité de l'anneau $\left(V[Y, Z]_{\mathcal{R}}\right)^{\dagger}$ unifie et généralise de nombreuses situations. Par exemple si l'on prend $m=n, h=0$, $R_{i, i}^{0}=1, R_{q, i}^{0}=0, q \neq i$ on trouve la noethérianité de l'article [17] qui est le point de départ de la division non commutative dans le cas ultramétrique et le cas le plus simple.

\subsection{Noethérianité des anneaux $D_{A^{\dagger} / V}^{\dagger, h}$}

Les ensembles $\mathcal{R}$ précédents sont formés de polynômes et pour pouvoir appliquer le théorème de division 5.2.7 il faut pouvoir relever l'action des opérateurs d'ordre fini sur les générateurs d'une algèbre $A^{\dagger}$ en des polynômes. Ceci nous conduit à la définition générale suivante :

Définition 5.3.1. Soit $\mathcal{X}^{\dagger}=\left(X, \mathcal{O}_{\mathcal{X}^{\dagger} / R}\right)$ un schéma $\dagger$-adique sur un couple $(R, I)$ pour un anneau $R$ noethérien. On dira qu'un ouvert $U$ de l'espace topologique $X$ a la propriété (diff-alg) si c'est un ouvert †-adique dont l'algèbre $A^{\dagger}$ est isomorphe au complété †-adique d'une $R$-algèbre $A$ lisse dont le A-module des formes différentielles $\Omega_{A / R}$ est libre. On dira qu'un système de coordonnées $x=\left(x_{1}, \ldots, x_{n}\right)$ de $A^{\dagger}$ est algébrique s'il provient d'un système de coordonnées de A.

Proposition 5.3.2. Soit $\mathcal{X}^{\dagger}=\left(X, \mathcal{O}_{\mathcal{X}^{\dagger} / R}\right)$ un schéma $\dagger$-adique lisse, alors tout point de $X$ admet un système fondamental de voisinages d'ouverts ayant la propriété (diff-alg).

Démonstration. C'est une conséquence directe du théorème des relèvements algébriques lisses ([1], [7]). En effet en vertu de ce théorème l'algèbre $A^{\dagger}$ d'un ouvert †-adique affine $U$ est isomorphe à l'algèbre $(A)^{\dagger}$ pour une $R$-algèbre lisse $A$ qui est donc de type fini sur $R$ et dont le $A$-module des différentielles $\Omega_{A / R}$ est donc projectif. Tout point de $U$ admet un système fondamental d'ouverts principaux de $U$ qui ont la propriété (diff-alg).

Revenons à la situation d'un couple $(V, \mathfrak{m})$ et d'un schéma †-adique $\mathcal{X}^{\dagger}=$ $\left(X, \mathcal{O}_{\mathcal{X}^{\dagger} / V}\right)$ lisse sur $V$. Soit $U$ un ouvert de $X$ ayant la propriété (diff-alg) et $A$ un relèvement lisse sur $V$ de l'anneau $\Gamma\left(U, \mathcal{O}_{X / k}\right)$. Soit

$$
V\left[Y_{1}, \ldots, Y_{m}\right] \rightarrow A \rightarrow 0
$$

une présentation de $A$ et $x=\left(x_{1}, \ldots, x_{n}\right)$ un système de coordonnées de $A$. 
Pour un entier $h \geq 0$ considérons un ensemble $\mathcal{R}:=\mathcal{R}_{m, n, h}$ de polynômes $R_{q, i}^{k} \in V[Y], 1 \leq q \leq m, 1 \leq i \leq n, 1 \leq k \leq p^{h}$ qui relèvent les éléments $\Delta_{i}^{k}\left(y_{q}\right)$ de l'algèbre $A$ où $y_{q}, 1 \leq q \leq m$ sont générateurs de $A$, images de $Y_{q}$ par (*). Nous pouvons donc considérer les anneaux $V[Y, Z]_{\mathcal{R}}$ et $\left(V[Y, Z]_{\mathcal{R}}\right)^{\dagger}$.

Proposition 5.3.3. Le morphisme défini par $Y_{q} \mapsto y_{q}, 1 \leq q \leq m$ et $Z_{i}^{j} \mapsto$ $\Delta_{i}^{p^{j}}, 1 \leq i \leq n, 0 \leq j \leq h$

$\Phi_{\mathcal{R}}:$

$$
V[Y, Z]_{\mathcal{R}} \rightarrow D_{A / V}^{<\infty, h}
$$

est un morphisme surjectif d'anneaux qui se prolonge en un morphisme d'anneaux

$\Phi_{\mathcal{R}}^{\dagger}:$

$$
\left(V[Y, Z]_{\mathcal{R}}\right)^{\dagger} \rightarrow D_{(A)^{\dagger} / V}^{\dagger, h}
$$

qui est surjectif.

Démonstration. Le morphisme $\Phi_{\mathcal{R}}$ est compatible au commutateur par construction ce qui entraîne que c'est un morphisme d'anneaux qui est surjectif de façon évidente. Soit

$$
P=\sum_{\alpha \in \mathbb{N}^{m}, \beta \in\left(\mathbb{N}^{n}\right)^{h+1}} a_{\alpha, \beta} Y^{\alpha} Z^{\beta}
$$

un élément de l'anneau $\left(V[Y, Z]_{\mathcal{R}}\right)^{\dagger}$, alors en vertu de la majoration du théorème 2.6.10, c'est le point, la série

$$
\Phi_{\mathcal{R}}^{\dagger}(P):=\sum_{\alpha \in \mathbb{N}^{n}, \beta \in\left(\mathbb{N}^{n}\right)^{h+1}} a_{\alpha, \beta} y^{\alpha}(\Delta)^{\beta}
$$

opère sur $(A)^{\dagger}$. D'autre part cette série a la propriété du degré de la définition 2.6.5. C'est donc un élément de l'anneau $D_{(A)^{\dagger} / V}^{\dagger, h}$, ce qui définit le morphisme $\Phi_{\mathcal{R}}^{\dagger}$ qui est $V$-linéaire. Reste à voir que c'est un morphisme d'anneaux. Si $P_{1}, P_{2}$ sont deux éléments de $\left(V[Y, Z]_{\mathcal{R}}\right)^{\dagger}$ il suffit de montrer que l'action de $\Phi_{\mathcal{R}}^{\dagger}\left(P_{2} P_{1}\right)$ sur $(A)^{\dagger}$ est égale à l'action de $\Phi_{\mathcal{R}}^{\dagger}\left(P_{2}\right) \circ \Phi_{\mathcal{R}}^{\dagger}\left(P_{1}\right)$ sur $(A)^{\dagger}$. Autrement dit si $f$ est un élément de $(A)^{\dagger}$ il faut montrer que les éléments $\Phi_{\mathcal{R}}^{\dagger}\left(P_{2} P_{1}\right)(f), \Phi_{\mathcal{R}}^{\dagger}\left(P_{2}\right) \circ \Phi_{\mathcal{R}}^{\dagger}\left(P_{1}\right)(f)$ de $(A)^{\dagger}$ sont égaux ou de façon équivalente, par séparation $\mathfrak{m}$-adique, qu'ils sont égaux dans $(A)^{\wedge}$ et ceci résulte du fait que le morphisme $\Phi_{\mathcal{R}}^{\wedge}$, complété du morphisme $\Phi_{\mathcal{R}}$, est un morphisme d'anneaux.

Le morphisme $\Phi_{\mathcal{R}}^{\dagger}$ est alors un morphisme d'anneaux qui est surjectif en vertu du théorème du symbole total 3.1.2 pour les opérateurs différentiels $p$-adiques d'échelon $h$.

On rappelle que l'on a posé

$$
\left(\Delta_{x}^{p^{j}}\right)^{\beta^{j}}:=\left(\Delta_{1}^{p^{j}}\right)^{\beta_{1}^{j}} \ldots\left(\Delta_{n}^{p^{j}}\right)^{\beta_{n}^{h}}, \quad(\Delta)^{\beta}:=\left(\Delta_{x}\right)^{\beta^{0}} \ldots\left(\Delta_{x}^{p^{h}}\right)^{\beta^{h}} .
$$


Remarque 5.3.4. En fait si l'on choisit une division dans l'algèbre $(V[Y])^{\dagger}$ par une base de division $B_{J}([13$, Thm.2.3.4]) de l'idéal $J$ provenant du noyau de la présentation $(*)$, le théorème de continuité de la division ([13, 2.3.4]) et le théorème du symbole total 3.1 .2 pour les opérateurs différentiels $p$-adique d'échelon $h$ définissent une rétraction $V$-linéaire du morphisme $\Phi_{\mathcal{R}}^{\dagger}$ dont le noyau est engendré par l'idéal bilatère engendré les éléments

$$
\left(\left(Z_{i}^{j}\right)^{p}-\frac{p^{j+1} !}{\left(p^{j} !\right)^{p}} Z_{i}^{j+1}, 1 \leq i \leq n, 0 \leq j \leq h-1\right)
$$

et la base de division $B_{J}$.

Corollaire 5.3.5. Les anneaux

$$
D_{(A)^{\dagger} / V}^{\dagger, h}, D_{(A)^{\dagger} / V}^{\dagger, h}\left[\Delta_{1}^{p^{h+1}}, \ldots, \Delta_{i}^{p^{h+1}}\right], \quad 1 \leq i \leq n,
$$

sont noethériens.

Démonstration. En effet ce sont des quotients des anneaux

$$
\left(V[Y, Z]_{\mathcal{R}}\right)^{\dagger}, \quad\left(V[Y, Z]_{\mathcal{R}}\right)^{\dagger}\left[Z_{1}^{p^{h+1}}, \ldots, Z_{i}^{p^{h+1}}\right]
$$

qui sont noethériens en vertu du paragraphe précédent.

Théorème 5.3.6. Soit $\left(X, \mathcal{O}_{\mathcal{X}^{\dagger} / V}\right)$ un †-schéma $V$-lisse et $U$ un ouvert ayant la propriété (diff-alg) d'algèbre $A^{\dagger}$ munie de coordonnées $x=\left(x_{1}, \ldots, x_{n}\right)$ algébriques et $h \geq 0$ un entier, alors les anneaux

$$
D_{A^{\dagger} / V}^{\dagger, h}, D_{A^{\dagger} / V}^{\dagger, h}\left[\Delta_{1}^{p^{h+1}}, \ldots, \Delta_{i}^{p^{h+1}}\right]
$$

pour $1 \leq i \leq n$ sont noethériens.

Démonstration. En effet l'anneau $D_{A^{\dagger} / V}^{\dagger, h}$ est isomorphe à l'anneau $D_{(A)^{\dagger} / V}^{\dagger, h}$ et l'anneau $D_{A^{\dagger} / V}^{\dagger, h}\left[\Delta_{1}^{p^{h+1}}, \ldots, \Delta_{i}^{p^{h+1}}\right]$ est isomorphe à l'anneau

$$
D_{(A)^{\dagger} / V}^{\dagger, h}\left[\Delta_{1}^{p^{h+1}}, \ldots, \Delta_{i}^{p^{h+1}}\right]
$$

pour une $V$-algèbre lisse $A$ munie de coordonnées. D'où le théorème 5.3.6.

Remarque 5.3.7. Nous avons déjà considéré des relèvements $\mathcal{R}$ dans la démonstration du théorème de l'opérateur différentiel d'un symbole totale dans l'article ([13, Thm. 6.1]). 


\section{Platitude et cohérence du faisceau $\mathcal{D}_{\mathcal{X}^{\dagger} / K}^{\dagger}$}

\subsection{Platitude}

Nous allons déduire de nombreuses conséquences de la noethérianité précédente en utilisant du critère de platitude locale 1.0.3.

Théorème 6.1.1. Soient $\mathcal{X}^{\dagger}:=\left(X, \mathcal{O}_{\mathcal{X}^{\dagger} / V}\right)$ un schéma $\dagger$-adique lisse sur $V$ et $h \geq 0$, alors l'extension

$$
\mathcal{D}_{\mathcal{X}^{\dagger} / V}^{<\infty, h} \rightarrow \mathcal{D}_{\mathcal{X}^{\dagger} / V}^{\dagger, h}
$$

est plate.

Démonstration. Il suffit de montrer pour un ouvert $U$ ayant la propriété (diff-alg) d'algèbre $A^{\dagger}$ que l'extension

$$
D_{A^{\dagger} / V}^{h} \rightarrow D_{A^{\dagger} / V}^{\dagger, h}
$$

est plate. En vertu du théorème 5.3.6 l'anneau $D_{A^{\dagger} / V}^{\dagger, h}$ est noethérien. D'autre part en vertu du théorème 4.1.1 l'idéal $\mathfrak{m}$ est contenu dans le radical de $D_{A^{\dagger} / V}^{\dagger, h}$. Le morphisme

$$
D_{A^{\dagger} / V}^{h} \rightarrow D_{A^{\dagger} / V}^{\dagger, h}
$$

induit un isomorphisme modulo $\mathfrak{m}^{s}$ pour tout $s \geq 1$. Comme l'anneau $D_{A^{\dagger} / V}^{h}$ est aussi noethérien il résulte du critère de platitude local 1.0.3 que l'extension précédente est elle même plate.

Corollaire 6.1.2. Soit $\mathcal{X}^{\dagger}:=\left(X, \mathcal{O}_{\mathcal{X}^{\dagger} / V}\right)$ un schéma $\dagger$-adique lisse sur $V$, alors l'extension

$$
\mathcal{D}_{\mathcal{X}^{\dagger} / V} \rightarrow \mathcal{D}_{\mathcal{X}^{\dagger} / V}^{\dagger}
$$

est plate ainsi donc que l'extension composée

$$
\mathcal{O}_{\mathcal{X}^{\dagger} / V} \rightarrow \mathcal{D}_{\mathcal{X}^{\dagger} / V} \rightarrow \mathcal{D}_{\mathcal{X}^{\dagger} / V}^{\dagger}
$$

Démonstration. Il suffit de montrer pour un ouvert $U$ ayant la propriété (diff-alg) d'algèbre $A^{\dagger}$ que l'extension

$$
D_{A^{\dagger} / V} \rightarrow D_{A^{\dagger} / V}^{\dagger}
$$

est plate. Il suffit de montrer pour tout idéal de type fini $J$ de $D_{A^{\dagger} / V}$ que le morphisme

$$
D_{A^{\dagger} / V}^{\dagger} \otimes_{D_{A^{\dagger} / V}} J \rightarrow D_{A^{\dagger} / V}^{\dagger}
$$

est injectif. 
Soient $B_{1}, \ldots, B_{s}$ un système de générateurs de l'idéal $J$ et $P_{1}, \ldots, P_{s}$ des opérateurs de $D_{A^{\dagger} / V}^{\dagger}$ tels que $P_{1} B_{1}+\cdots+P_{s} B_{s}$ est nul dans l'anneau $D_{A^{\dagger} / V}^{\dagger}$. Il existe un entier $h \geq 0$ tel que $B_{1}, \ldots, B_{s}$ appartiennent à $D_{A^{\dagger} / V}^{<\infty, h}$ et $P_{1}, \ldots, P_{s}$ appartiennent à $D_{A^{\dagger} / V}^{\dagger, h}$. Soit $J_{h}$ l'idéal de $D_{A^{\dagger} / V}^{\dagger, h}$ engendré par $B_{1}, \ldots, B_{s}$. On a alors un diagramme commutatif

$$
\begin{gathered}
D_{A^{\dagger} / V}^{\dagger, h} \otimes_{D_{A^{\dagger} / V}^{<\infty, h}} J_{h} \rightarrow D_{A^{\dagger} / V}^{\dagger, h} \\
\downarrow \\
D_{A^{\dagger} / V}^{\dagger} \otimes_{D_{A^{\dagger} / V}} J \rightarrow D_{A^{\dagger} / V}^{\dagger}
\end{gathered}
$$

et l'élément $\sum P_{i} \otimes B_{i}$ du produit tensoriel $D_{A^{\dagger} / V}^{\dagger} \otimes_{D_{A^{\dagger} / V}} J$ provient de l'élément $\sum P_{i} \otimes B_{i}$ du produit tensoriel $D_{A^{\dagger} / V}^{\dagger, h} \otimes_{D_{A^{\dagger} / V}^{<\infty, h}} J_{h}$. En vertu de la platitude du théorème précédent cet élément est nul et donc le morphisme du corollaire est injectif.

Remarque 6.1.3. Le lecteur prendra garde que les extensions précédentes $\mathcal{D}_{\mathcal{X}^{\dagger} / V} \rightarrow \mathcal{D}_{\mathcal{X}^{\dagger} / V}^{\dagger}, \mathcal{D}_{\mathcal{X}^{\dagger} / K} \rightarrow \mathcal{D}_{\mathcal{X}^{\dagger} / K}^{\dagger}$ sont plates mais non fidèlement plates.

Théorème 6.1.4. Soit $\mathcal{X}^{\dagger}:=\left(X, \mathcal{O}_{\mathcal{X}^{\dagger} / V}\right)$ un $\dagger$-adique schéma $V$-lisse et $U$ un ouvert ayant la propriété (diff-alg) d'algèbre $A^{\dagger}$, alors pour tout $h \geq 0$ l'extension

$$
D_{A^{\dagger} / V}^{\dagger, h} \rightarrow D_{A^{\wedge} / V}^{\wedge, h}
$$

est fidèlement plate.

Démonstration. Sous l'hypothèse précédente l'anneau $D_{A^{\dagger} / V}^{\dagger, h}$ est noethérien et l'anneau $D_{A^{\wedge} / V}^{\wedge, h}$ est idéalement séparé pour la topologie m-adique. Comme ces deux anneaux ont même réduction modulo $\mathfrak{m}^{h}$ pour tout $h \geq 0$ il résulte du critère de platitude locale 1.0.3 que l'extension est plate.

Soit $M$ un $D_{A^{\dagger} / V}^{\dagger, h}$-module de type fini tel que $D_{A^{\wedge} / V}^{\wedge, h} \otimes_{D_{A^{\dagger} / V}^{\dagger, h}} M$ est nul, alors sa réduction modulo $\mathfrak{m}$ est nulle. Comme en vertu du théorème 4.1.1 l'idéal $\mathfrak{m}$ est contenu dans la radical de $D_{A^{\dagger} / V}^{\dagger, h}$, il résulte du lemme de Nakayama que $M$ est nul et l'extension du théorème précédent est fidèlement plate.

Corollaire 6.1.5. Soit $\left(X, \mathcal{O}_{\mathcal{X}^{\dagger} / V}\right)$ un $\dagger$-schéma $V$-lisse pour tout $h \geq 0$ les extensions

$$
\mathcal{D}_{\mathcal{X}^{\dagger} / V}^{\dagger, h} \rightarrow \mathcal{D}_{\mathcal{X} / V}^{\wedge, h}, \mathcal{D}_{\mathcal{X}^{\dagger} / V}^{\dagger} \rightarrow \mathcal{D}_{\mathcal{X} / V}^{\dagger}
$$

sont fidèlement plates. 
Démonstration. En effet la première extension est fidèlement plate en vertu du théorème précédent et la seconde extension est fidèlement plate par passage à la limite inductive en $h$.

Théorème 6.1.6. Soit $\mathcal{X}^{\dagger}:=\left(X, \mathcal{O}_{\mathcal{X}^{\dagger} / V}\right)$ un $\dagger$-adique schéma $V$-lisse et $U$ un ouvert ayant la propriété (diff-alg) d'algèbre $A^{\dagger}$, alors pour tout $h \geq 0$ l'extension

$$
D_{A^{\dagger} / K}^{\dagger, h} \rightarrow D_{A^{\dagger} / K}^{\dagger, h+1}
$$

est plate où $D_{A^{\dagger} / K}^{\dagger, h}:=D_{A^{\dagger} / V}^{\dagger, h} \otimes_{V} K$.

Démonstration. Soit $\left(x_{1}, \ldots, x_{n}\right)$ un système de coordonnées algébriques au-dessus de $U$, considérons les extensions

$$
D_{A^{\dagger} / V}^{\dagger, h} \rightarrow D_{A^{\dagger} / V}^{\dagger, h}\left[\Delta_{1}^{p^{h+1}}, \ldots, \Delta_{n}^{p^{h+1}}\right] \rightarrow D_{A^{\dagger} / V}^{\dagger, h+1} .
$$

L'anneau intermédiaire est noethérien en vertu du théorème 5.3.6 et donc la seconde extension est plate en vertu du critère de platitude locale 1.0.3, les deux anneaux ayant même réduction modulo $\mathfrak{m}^{s}$ pour tout $s \geq 1$. La première extension n'est pas plate mais le changement de base $V \rightarrow K$ induit un isomorphisme

$$
D_{A^{\dagger} / K}^{\dagger, h} \simeq D_{A^{\dagger} / V}^{\dagger, h}\left[\Delta_{1}^{p^{h+1}}, \ldots, \Delta_{n}^{p^{h+1}}\right] \otimes_{V} K
$$

D'où le théorème 6.1.6 par stabilité de la platitude par composition.

Remarque 6.1.7. La démonstration précédente en beaucoup plus simple montre que l'extension $D_{A^{\wedge} / K}^{\wedge, h} \rightarrow D_{A^{\wedge} / K}^{\wedge, h+1}$ est plate pour tout schéma formel affine complet lisse d'algèbre $A^{\wedge}$ ayant la propriété (diff) et pas seulement (diff-alg).

Corollaire 6.1.8. Soit $\mathcal{X}^{\dagger}:=\left(X, \mathcal{O}_{\mathcal{X}^{\dagger} / V}\right)$ un $\dagger$-schéma sur $V$ lisse, alors pour tout $h \geq 0$, les extensions

$$
\mathcal{D}_{\mathcal{X}^{\dagger} / K}^{\dagger, h} \rightarrow \mathcal{D}_{\mathcal{X}^{\dagger} / K}^{\dagger, h+1}, \quad \mathcal{D}_{\mathcal{X}^{\dagger} / K}^{\dagger, h} \rightarrow \mathcal{D}_{\mathcal{X}^{\dagger} / K}^{\dagger}
$$

sont plates où $\mathcal{D}_{\mathcal{X}^{\dagger} / K}^{\dagger, h}:=\mathcal{D}_{\mathcal{X}^{\dagger} / V}^{\dagger, h} \otimes_{V} K$.

Démonstration. La première extension est plate en vertu du théorème précédent. D'autre part on a l'isomorphisme :

$$
\lim _{\rightarrow h} \mathcal{D}_{\mathcal{X}^{\dagger} / K}^{\dagger, h} \simeq \mathcal{D}_{\mathcal{X}^{\dagger} / K}^{\dagger}
$$

qui montre que la seconde extension est aussi plate parce la platitude est stable par composition. 


\subsection{Cohérence}

Nous allons appliquer la méthode de la théorie des faisceaux cohérents qui ne sont pas noethériens, dont l'exemple type est le faisceau $\mathcal{D}_{X / k}$, pour montrer que le faisceau $\mathcal{D}_{\mathcal{X}^{\dagger} / K}^{\dagger}$ est cohérent.

Théorème 6.2.1. Soit $\mathcal{X}^{\dagger}=\left(X, \mathcal{O}_{\mathcal{X}^{\dagger} / V}\right)$ un $\dagger$-schéma sur $V$ lisse, alors pour tout $h \geq 0$, les faisceaux d'anneaux

$$
\mathcal{D}_{\mathcal{X}^{\dagger} / V}^{\dagger, h}, \quad \mathcal{D}_{\mathcal{X}^{\dagger} / K}^{\dagger, h}, \quad \mathcal{D}_{\mathcal{X}^{\dagger} / K}^{\dagger}
$$

sont cohérents (à gauche et à droite).

Nous allons d'abord montrer le critère de cohérence général suivant.

Proposition 6.2.2. Soit $X$ un espace topologique et $\mathcal{A}_{X}$ un faisceaux d'anneaux unitaires sur $X$ qui admet une base $\mathcal{B}$ de la topologie telle que:

1) pour tout ouvert $U$ de la base $\mathcal{B}$, la cohomologie $H^{i}\left(U, \mathcal{A}_{X}\right)$ est triviale pour $i \geq 1$,

2) pour tout ouvert $U$ de la base $\mathcal{B}$ l'anneau $\Gamma\left(U, \mathcal{A}_{X}\right)$ est noethérien (à gauche ou à droite),

3) pour tout ouvert $U$ de la base $\mathcal{B}$, tout point de $U$ admet un système fondamental formé d'ouverts appartenant à la base $\mathcal{B}$, ou de façon équivalente l'ouvert $U$ admet une base $\mathcal{B}^{\prime}:=\mathcal{B}_{U}^{\prime}$ formée d'ouverts appartenant à la base $\mathcal{B}, W \subset U$ de tel que l'extension $\Gamma\left(U, \mathcal{A}_{X}\right) \rightarrow$ $\Gamma\left(W, \mathcal{A}_{X}\right)$ induite par la restriction est plate.

Alors le faisceau $\mathcal{A}_{X}$ est cohérent (à gauche ou à droite).

Démonstration. La question est locale, soit $U$ un ouvert de la base $\mathcal{B}$ et

$$
\left(\mathcal{A}_{U}\right)^{r} \rightarrow \mathcal{A}_{U}
$$

un morphisme de $\mathcal{A}_{U}$-modules au-dessus d'un ouvert $U$. D'où un morphisme de $\Gamma\left(U, \mathcal{A}_{U}\right)$-modules

$$
\left(\Gamma\left(U, \mathcal{A}_{U}\right)\right)^{r} \rightarrow \Gamma\left(U, \mathcal{A}_{U}\right)
$$

La condition 2) de noethérianité fournit une suite

$$
\left(\Gamma\left(U, \mathcal{A}_{U}\right)\right)^{q} \rightarrow\left(\Gamma\left(U, \mathcal{A}_{U}\right)\right)^{r} \rightarrow \Gamma\left(U, \mathcal{A}_{U}\right) .
$$

qui exacte au milieu. D'où un complexe de faisceaux

$$
0 \rightarrow\left(\mathcal{A}_{U}\right)^{q} \rightarrow\left(\mathcal{A}_{U}\right)^{r} \rightarrow \mathcal{A}_{U} \rightarrow 0
$$


Il s'agit de montrer que la cohomologie du milieu de ce complexe est triviale. Mais cette cohomologie de faisceaux est le faisceau associé au préfaisceau qui à un ouvert $W \subset U$ associe l'hypercohomologie $H^{1}\left(W, \mathcal{A}^{\bullet}\right)$ du complexe $(*)$ précédent. Mais en vertu de la condition 1) d'acyclicité cette hypercohomologie est la cohomologie du milieu du complexe

$$
0 \rightarrow\left(\Gamma\left(W, \mathcal{A}_{U}\right)\right)^{q} \rightarrow\left(\Gamma\left(W, \mathcal{A}_{U}\right)\right)^{r} \rightarrow \Gamma\left(W, \mathcal{A}_{U}\right) \rightarrow 0 .
$$

Pour l'ouvert $U$ elle est triviale par construction et pour un ouvert $W \subset U$ de la base $\mathcal{B}^{\prime}$ elle reste triviale par platitude. La cohomologie du milieu du complexe de faisceaux $(*)$ est nulle. D'où le critère de cohérence 6.2.2

Démonstration du théorème 6.2.1. Soit la base $\mathcal{B}$ de $X$ formée des ouverts $U$ ayant la propriété (diff-alg) et $\mathcal{B}^{\prime}$ la base de $U$ formée par les ouverts $W$ principaux, alors les bases précédentes ont les propriétés 1), 2), 3 ) de la proposition précédente pour le faisceau $\mathcal{D}_{\mathcal{X}^{\dagger} / V}^{\dagger, h}$ pour tout $h \geq 0$. En effet l'acyclicité est le théorème 3.2.3, la noethérianité est le théorème 5.3.6 et la platitude résulte du critère locale de platitude 1.0.3. Il en résulte que le faisceau $\mathcal{D}_{\mathcal{X}^{\dagger} / V}^{\dagger, h}$ est cohérent en vertu du critère de cohérence 6.2.2 ainsi que le faisceau $\mathcal{D}_{\mathcal{X}^{\dagger} / K}^{\dagger, h}$.

Pour passer au faisceau $\mathcal{D}_{\mathcal{X}^{\dagger} / K}^{\dagger}$ nous rappelons la proposition classique :

Proposition 6.2.3. Soient un espace topologique $X$ localement noethérien et un système inductif

$$
u_{h}: \mathcal{A}_{X}^{h} \rightarrow \mathcal{A}_{X}^{h+1}, \quad h \in \mathbb{N},
$$

de faisceaux d'anneaux (à gauche ou à droite) cohérents tel que les extensions $u_{h}$ sont plates, alors la limite inductive

$$
\mathcal{A}_{X}:=\lim _{\rightarrow h} \mathcal{A}_{X}^{h}
$$

est un faisceau d'anneaux (à gauche ou à droite) cohérent.

Démonstration. Soit un morphisme de $\mathcal{A}_{U}$-modules

$$
\left(\mathcal{A}_{U}\right)^{r} \stackrel{G}{\rightarrow}\left(\mathcal{A}_{U}\right)^{s}
$$

au voisinage $U$ d'un point de $X$. Quitte à prendre un voisinage ouvert plus petit en vertu de la noethérianité locale on peut supposer que les coefficients de la matrice $G$ proviennent d'éléments de $\Gamma\left(U, \mathcal{A}_{U}^{h}\right)$ pour un $h$ assez grand. Quitte à diminuer encore $U$ en vertu de la cohérence il existe une suite

$$
\left(\mathcal{A}_{U}^{h}\right)^{r} \rightarrow\left(\mathcal{A}_{U}^{h}\right)^{s} \stackrel{G^{h}}{\rightarrow}\left(\mathcal{A}_{U}^{h}\right)^{t}
$$


qui exacte au milieu. D'où par platitude une suite

$$
\left(\mathcal{A}_{U}\right)^{r} \rightarrow\left(\mathcal{A}_{U}\right)^{s} \stackrel{G}{\rightarrow}\left(\mathcal{A}_{U}\right)^{t}
$$

qui est exacte au milieu et exprime que le faisceau $\mathcal{A}_{X}$ est cohérent.

En vertu du théorème 6.1.6 et la proposition précédente on trouve que le faisceau $\mathcal{D}_{\mathcal{X}^{\dagger} / K}^{\dagger}$ est cohérent. D'où le théorème 6.2.1.

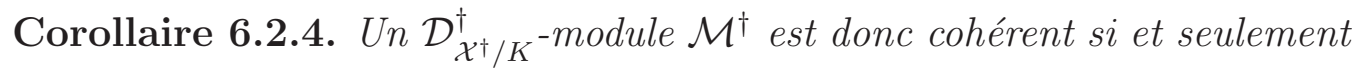
s'il est localement de présentation finie.

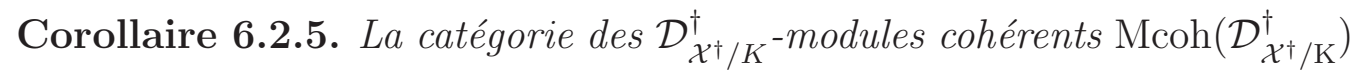
est abélienne et la catégorie des complexes $\mathrm{D}_{\mathrm{coh}}^{\mathrm{b}}\left(\mathcal{D}_{\mathcal{X}^{\dagger} / \mathrm{K}}^{\dagger}\right)$ de $\mathcal{D}_{\mathcal{X}^{\dagger} / K^{-}}^{\dagger}$-modules à cohomologie bornée et cohérente est triangulée.

Remarque 6.2.6. Bien entendu tous les résultats précédents valent pour les faisceaux $\mathcal{D}_{\mathcal{X} / V}^{<\infty, h}, \mathcal{D}_{\mathcal{X} / V}, \mathcal{D}_{\mathcal{X} / V}^{\wedge, h}, \mathcal{D}_{\mathcal{X} / K}^{\wedge, h}, \mathcal{D}_{\mathcal{X} / V}^{\dagger}, \mathcal{D}_{\mathcal{X} / K}^{\dagger}$ avec des démonstrations beaucoup plus simples et aussi pour les faisceaux $\mathcal{D}_{X / k}^{<\infty, h}, \mathcal{D}_{X / k}$.

Remarque 6.2.7. 1) Nous savons démontrer que le faisceau $\mathcal{D}_{\mathcal{X}^{\dagger} / V}^{\dagger}$ est cohérent si $\operatorname{dim} X=1$, ce qui entraîne que la dimension homologique faible du faisceau $\mathcal{D}_{\mathcal{X}^{\dagger} / V}^{\dagger}$ est exactement 2 et donc que la dimension homologique faible du faisceau $\mathcal{D}_{\mathcal{X}^{\dagger} / K}^{\dagger}$ est bornée par 2.

2) Mais le faisceau $\mathcal{D}_{\mathcal{X}^{\dagger} / V}^{\dagger}$ n'est pas cohérent si $\operatorname{dim} X \geq 2$. Par exemple l'idéal de type fini $\left(\Delta_{x}, \Delta_{y}\right)$ n'est pas localement de présentation finie pour $X=\operatorname{Spec}(k[x, y])$ de relèvement $(V[x, y])^{\dagger}$.

En effet pour $l \geq 1$ posons

$$
\left(u_{l}, v_{l}\right):=\left(\Delta_{y}^{p^{l}}\left(\Delta_{x}^{p^{l-1}} \ldots \Delta_{x}\right), \Delta_{x}^{p^{l}}\left(\Delta_{y}^{p^{l-1}} \ldots \Delta_{y}\right)\right) .
$$

On a la relation $u_{l} \Delta_{x}-v_{l} \Delta_{y}=0$ qui définit un élément de

$$
\operatorname{Tor}_{\mathrm{D}_{(\mathrm{V}[\mathrm{x}, \mathrm{y}])^{\dagger} / \mathrm{V}}^{\dagger}}^{1,0}\left(\mathrm{D}_{(\mathrm{V}[\mathrm{x}, \mathrm{y}])^{\dagger} / \mathrm{V}}^{\dagger}, \mathrm{D}_{(\mathrm{V}[\mathrm{x}, \mathrm{y}])^{\dagger} / \mathrm{V}}^{\dagger, 0} /\left(\Delta_{\mathrm{x}}, \Delta_{\mathrm{y}}\right)\right) \text {. }
$$

On a la nullité $p^{l+1}\left(u_{l}, v_{l}\right)=0$ dans le module de torsion précédent mais $p^{l}\left(u_{l}, v_{l}\right) \neq 0$. La torsion du $D_{(V[x, y])^{\dagger} / V^{\dagger}}^{\dagger}$-module à gauche de torsion précédent est infinie ce qui montre qu'il n'est pas de type fini et ceci reste vrai pour tout ouvert affine principal. Ceci est dû aux relations du type

$$
\Delta_{y}^{p}\left(\Delta_{x}^{1}\right)^{p}-\Delta_{x}^{p}\left(\Delta_{y}^{1}\right)^{p}=0
$$

qui n'existent pas en dimension une. 
3) Comme nous l'avons déjà signalé dans l'article de recherche [11] tous les faisceaux précédents $\mathcal{D}_{\mathcal{X}^{\dagger} / V}^{\dagger, h}, \mathcal{D}_{\mathcal{X}^{\dagger} / K}^{\dagger, h}, \mathcal{D}_{\mathcal{X}^{\dagger} / V}^{\dagger}, \mathcal{D}_{\mathcal{X}^{\dagger} / K}^{\dagger}$ sont de dimension homologique plate finie. En effet les réductions modulo $\mathfrak{m}$ des faisceaux $\mathcal{D}_{\mathcal{X}^{\dagger} / V}^{\dagger, h}$ sont de dimension homologique plate exactement égale à $2 \operatorname{dim} X$. Cela entraîne de la dimension homologique plate de $\mathcal{D}_{\mathcal{X}^{\dagger} / V}^{\dagger, h}$ est égale à $2 \operatorname{dim} X+1$ et celle des faisceaux $\mathcal{D}_{\mathcal{X}^{\dagger} / V}^{\dagger}, \mathcal{D}_{\mathcal{X}^{\dagger} / K}^{\dagger}$ est bornée par $2 \operatorname{dim} X+2$. On conjecture $([11])$ que la dimension homologique plate du faisceau $\mathcal{D}_{\mathcal{X}^{\dagger} / K}^{\dagger}$ est exactement $\operatorname{dim} X$ mais ceci n'est pas démontré à notre connaissance même en dimension une. Comme on l'a déjà signalé on sait seulement que cette dimension est bornée par 2 .

4) Tous les résultats précédents restent bien sûr valables dans la théorie formelle. Le faisceau $\mathcal{D}_{\mathcal{X} / V}^{\dagger}$ est cohérent si $\operatorname{dim} X=1$ et de dimension plate 2, mais n'est pas cohérent si $\operatorname{dim} X \geq 2$. Les faisceaux $\mathcal{D}_{\mathcal{X} / V}^{\wedge, h}, \mathcal{D}_{\mathcal{X} / K}^{\wedge, h}, \mathcal{D}_{\mathcal{X} / V}^{\dagger}, \mathcal{D}_{\mathcal{X} / K}^{\dagger}$ sont de dimension homologique plate finie. En effet les réductions modulo $\mathfrak{m}$ des faisceaux $\mathcal{D}_{\mathcal{X} / V}^{\wedge, h}$ sont de dimension homologique plate exactement égale à $2 \operatorname{dim} X$. Cela entraîne de la dimension homologique plate de $\mathcal{D}_{\mathcal{X} / V}^{\wedge, h}$ est égale à $2 \operatorname{dim} X+1$ et celle des faisceaux $\mathcal{D}_{\mathcal{X} / V}^{\dagger}, \mathcal{D}_{\mathcal{X} / K}^{\dagger}$ est bornée par $2 \operatorname{dim} X+2$. On conjecture ([11]) que la dimension homologique plate du faisceau $\mathcal{D}_{\mathcal{X} / K}^{\dagger}$ est exactement $\operatorname{dim} X$ mais ceci n'est pas démontré à notre connaissance même en dimension une. On sait seulement que cette dimension est bornée par 2 .

\section{La Cohomologie de de Rham $p$-adique et la Cohomo- logie de Monsky-Washnitzer}

\subsection{La cohomologie de de Rham $p$-adique}

Soit $\mathcal{X}^{\dagger}=:\left(X, \mathcal{O}_{\mathcal{X}^{\dagger} / V}\right)$ un schéma $\dagger$-adique lisse sur $V$ lisse. Nous rappelons la définition que nous avons proposée dans l'article fondamental de recherche ([11, page 303]) de la cohomologie de de Rham $p$-adique d'un $\mathcal{D}_{\mathcal{X}^{\dagger} / V^{-}}$ module qui nous a conduit à la théorie des équations différentielles $p$-adiques et à leur monodromie.

Définition 7.1.1. Soit $\mathcal{M}^{\dagger}$ un complexe de la catégorie $\mathrm{D}^{+}\left(\mathcal{D}_{\mathcal{X}^{\dagger} / \mathrm{V}}^{\dagger}\right)$, nous avons défini les espaces de cohomologie de de Rham p-adique de $\mathcal{M}^{\dagger}$ comme les espaces d'hypercohomologie du complexe $\mathbf{R} \mathcal{H}_{\mathcal{D}_{\mathcal{X}^{\dagger} / V}^{\dagger}}\left(\mathcal{O}_{\mathcal{X}^{\dagger} / V}, \mathcal{M}^{\dagger}\right)$ de $\mathbf{Z a -}$ riski :

$$
\operatorname{Ext}_{\mathcal{D}_{\mathcal{X}^{\dagger} / V}^{\dagger}}^{\bullet}\left(\mathcal{O}_{\mathcal{X}^{\dagger} / V}, \mathcal{M}^{\dagger}\right)
$$


c'est-à-dire la cohomologie du complexe $\mathbf{R} \operatorname{Hom}_{\mathcal{D}_{\mathcal{X}^{\dagger} / V}^{\dagger}}\left(\mathcal{O}_{\mathcal{X}^{\dagger} / V}, \mathcal{M}^{\dagger}\right)$, foncteur dérivé à droite du foncteur $\mathcal{M}^{\dagger} \mapsto \operatorname{Hom}_{\mathcal{D}_{\mathcal{X}^{\dagger} / V}^{\dagger}}\left(\mathcal{O}_{\mathcal{X}^{\dagger} / V}, \mathcal{M}^{\dagger}\right)$ exacte à gauche.

Soit $\mathcal{M}^{\dagger}$ un complexe de la catégorie $\mathrm{D}^{+}\left(\mathcal{D}_{\mathcal{X}^{\dagger} / \mathrm{K}}^{\dagger}\right)$, nous avons défini les espaces de cohomologie de de Rham p-adique de $\mathcal{M}^{\dagger}$ comme les espaces d'hypercohomologie du complexe $\mathbf{R} \mathcal{H}_{\mathcal{D}^{\prime}}^{\dagger}\left(\mathcal{O}_{\mathcal{X}^{\dagger} / K}, \mathcal{M}^{\dagger}\right)$ de Zariski :

$$
\operatorname{Ext}_{\mathcal{D}_{\mathcal{X}^{\dagger} / K}^{\dagger}}^{\bullet}\left(\mathcal{O}_{\mathcal{X}^{\dagger} / K}, \mathcal{M}^{\dagger}\right)
$$

c'est-à-dire la cohomologie du complexe $\mathbf{R} \operatorname{Hom}_{\mathcal{D}_{\mathcal{X}^{\dagger} / K}^{\dagger}}\left(\mathcal{O}_{\mathcal{X}^{\dagger} / K}, \mathcal{M}^{\dagger}\right)$, foncteur dérivé à droite du foncteur $\mathcal{M}^{\dagger} \mapsto \operatorname{Hom}_{\mathcal{D}_{\mathcal{X}^{\dagger} / K}^{\dagger}}\left(\mathcal{O}_{\mathcal{X}^{\dagger} / K}, \mathcal{M}^{\dagger}\right)$ exacte à gauche.

Les espaces de cohomologie de de Rham $p$-adique ne commute pas au changement de base $V \rightarrow K$, ce qui suggère de modifier la définition de la cohomologie sur les entiers.

\subsection{Le Théorème de comparaison entre la cohomologie de de Rham $p$-adique et la cohomologie de Monsky-Washnitzer}

Soit $X$ une variété algébrique affine lisse sur $k$, Monsky-Washnitzer ont défini les espaces de cohomologie $\mathrm{H}_{\mathrm{dR}}^{\bullet}(\mathrm{X} / \mathrm{K})$ comme la cohomologie du complexe de de Rham des formes différentielles séparées d'un relèvement lisse sur $V[16]$.

Théorème 7.2.1. Soient $X$ variété algébrique affine lisse sur $k$ et $\mathcal{X}^{\dagger}:=$ $\left(X, \mathcal{O}_{\mathcal{X}^{\dagger} / V}\right)$ un schéma $\dagger$-adique lisse sur $V$ qui relève $X$, alors il existe des isomorphismes canoniques

$$
\mathrm{H}_{\mathrm{dR}}^{\bullet}(\mathrm{X} / \mathrm{K}) \simeq \operatorname{Ext}_{\mathcal{D}_{\mathcal{X}^{\dagger} / \mathrm{K}}^{\dagger}}^{\bullet}\left(\mathcal{O}_{\mathcal{X}^{\dagger} / \mathrm{K}}, \mathcal{O}_{\mathcal{X}^{\dagger} / \mathrm{K}}\right)
$$

Démonstration. On peut supposer que $X$ est connexe de dimension $n$. Soit $\mathcal{T}_{\mathcal{X}^{\dagger} / K}$ le fibré tangent dual du faisceau des formes différentielles séparées :

$$
\mathcal{T}_{\mathcal{X}^{\dagger} / K}:=\mathcal{H o m}_{\mathcal{O}_{\mathcal{X}^{\dagger} / K}}\left(\Omega_{\mathcal{X}^{\dagger} / K}, \mathcal{O}_{\mathcal{X}^{\dagger} / K}\right)
$$

Considérons le complexe de Spencer $\operatorname{Spen}^{\bullet}\left(\mathcal{O}_{\mathcal{X}^{\dagger} / K}\right)$ défini par :

$$
\operatorname{Spen}^{\bullet}\left(\mathcal{O}_{\mathcal{X}^{\dagger} / K}\right):=0 \rightarrow \mathcal{D}_{\mathcal{X}^{\dagger} / K} \otimes_{\mathcal{O}_{\mathcal{X}^{\dagger} / K}} \bigwedge^{n} \mathcal{T}_{\mathcal{X}^{\dagger} / K} \rightarrow \cdots \rightarrow \mathcal{D}_{\mathcal{X}^{\dagger} / K} \rightarrow \mathcal{O}_{\mathcal{X}^{\dagger} / K} \rightarrow 0
$$


où $\bigwedge^{i} \mathcal{T}_{\mathcal{X}^{\dagger} / K}$ est l'algèbre extérieure du fibré tangent et la différentielle $\delta$ est définie par :

$$
\begin{aligned}
\delta\left(P \otimes v_{1}\right. & \left.\wedge \cdots \wedge v_{i}\right):=\sum_{j=1, \ldots, i}(-1)^{i-1} P v_{j} \otimes\left(v_{1} \wedge \cdots \wedge \hat{v}_{j} \wedge v_{i}\right)- \\
& -\sum_{1 \leq j \leq l \leq i}(-1)^{i+l} P \otimes\left(\left[v_{j}, v_{l}\right] \wedge v_{1} \wedge \cdots \wedge \hat{v}_{j} \cdots \wedge \hat{v}_{j} \cdots \wedge v_{i}\right)
\end{aligned}
$$

où $P$ est un opérateur différentiel d'ordre fini et les $v_{j}$ sont des vecteurs tangents. Si $\partial_{1}, \ldots, \partial_{n}$ est une base locale duale d'une base de formes différentielles séparées le complexe $\operatorname{Spen} \bullet\left(\mathcal{O}_{\mathcal{X} \dagger / K}\right)$ apparaît comme le complexe de Koszul construite à partir de l'isomorphisme de $\mathcal{D}_{\mathcal{X}^{\dagger} / K^{-}}$modules à gauche :

$$
\mathcal{D}_{\mathcal{X}^{\dagger} / K} /\left(\partial_{1}, \ldots, \partial_{n}\right) \simeq \mathcal{O}_{\mathcal{X}^{\dagger} / K}
$$

Par un calcul élémentaire on voit que la suite $\partial_{1}, \ldots, \partial_{n}$ est régulière. Ceci montre que le complexe de Spencer $\operatorname{Spen}^{\bullet}\left(\mathcal{O}_{\mathcal{X}^{\dagger} / K}\right)$ est cohomologiquement trivial et fournit une résolution de $\mathcal{D}_{\mathcal{X}^{\dagger} / K^{-}}$-module à gauche du fibré trivial $\mathcal{O}_{\mathcal{X}^{\dagger} / K}$ par des $\mathcal{D}_{\mathcal{X}^{\dagger} / K^{-m o d u l e s ~ a ̀ ~ g a u c h e ~ l o c a l e m e n t ~ l i b r e s ~ d e ~ t y p e ~ f i n i . ~ L e ~}}$ complexe

$$
\mathbf{R} \mathcal{H o m}_{\mathcal{D}_{\mathcal{X}^{\dagger} / K}}\left(\mathcal{O}_{\mathcal{X}^{\dagger} / K}, \mathcal{O}_{\mathcal{X}^{\dagger} / K}\right)
$$

se représente par le complexe

$$
\mathcal{H o m}_{\mathcal{D}_{\mathcal{X}^{\dagger} / K}}\left(\operatorname{Spen}^{\bullet}\left(\mathcal{O}_{\mathcal{X}^{\dagger} / K}\right), \mathcal{O}_{\mathcal{X}^{\dagger} / K}\right)
$$

qui est le complexe de de Rham des formes différentielles séparées :

$$
0 \rightarrow \mathcal{O}_{\mathcal{X}^{\dagger} / K} \rightarrow \Omega_{\mathcal{X}^{\dagger} / K}^{1} \cdots \rightarrow \Omega_{\mathcal{X}^{\dagger} / K}^{n} \rightarrow 0 .
$$

En vertu du théorème d'acyclicité de Meredith [15] les groupes de cohomologie des faisceaux $\Omega_{\mathcal{X}^{\dagger} / K}^{i}$ sont nuls et le complexe

$$
\mathbf{R} \Gamma\left(X, \mathbf{R} \mathcal{H}_{o m_{\mathcal{X}^{\dagger} / K}}\left(\mathcal{O}_{\mathcal{X}^{\dagger} / K}, \mathcal{O}_{\mathcal{X}^{\dagger} / K}\right)\right)
$$

se représente par le complexe

$$
0 \rightarrow \Gamma\left(X, \mathcal{O}_{\mathcal{X}^{\dagger} / K}\right) \rightarrow \cdots \rightarrow \Gamma\left(X, \Omega_{\mathcal{X}^{\dagger} / K}^{n}\right) \rightarrow 0
$$

dont la cohomologie est par définition la cohomologie de Monsky-Washnitzer [16].

Proposition 7.2.2. Soit $\mathcal{M}^{\dagger}$ un $\mathcal{D}_{\mathcal{X}^{\dagger} / K^{-}}^{\dagger}$ module à gauche, alors il existe un isomorphisme canonique

$$
\mathbf{R} \mathcal{H o m}_{\mathcal{D}_{\mathcal{X}^{\dagger} / K}}\left(\mathcal{O}_{\mathcal{X}^{\dagger} / K}, \mathcal{M}^{\dagger}\right) \simeq \mathbf{R} \mathcal{H}_{\mathcal{D}^{\prime}}^{\dagger}\left(\mathcal{D}_{\mathcal{X}^{\dagger} / K}^{\dagger} \otimes_{\mathcal{D}_{\mathcal{X}^{\dagger} / K}} \mathcal{O}_{\mathcal{X}^{\dagger} / K}, \mathcal{M}^{\dagger}\right) .
$$


Démonstration. En effet la formule de changement de bases montre l'isomorphisme

$$
\mathbf{R} \mathcal{H o m}_{\mathcal{D}_{\mathcal{X}^{\dagger} / K}}\left(\mathcal{O}_{\mathcal{X}^{\dagger} / K}, \mathcal{M}^{\dagger}\right) \simeq \mathbf{R} \mathcal{H o m}_{\mathcal{D}_{\mathcal{X}^{\dagger} / K}^{\dagger}}\left(\mathcal{D}_{\mathcal{X}^{\dagger} / K}^{\dagger} \stackrel{\mathbf{L}}{\otimes_{\mathcal{D}_{\mathcal{X}^{\dagger} / K}}} \mathcal{O}_{\mathcal{X}^{\dagger} / K}, \mathcal{M}^{\dagger}\right) .
$$

Mais en vertu du corollaire 6.1.2 l'extension $\mathcal{D}_{\mathcal{X}^{\dagger} / K} \rightarrow \mathcal{D}_{\mathcal{X}^{\dagger} / K}^{\dagger}$ est plate. D'où l'isomorphisme de la proposition.

Proposition 7.2.3. Le morphisme canonique

$$
\mathcal{D}_{\mathcal{X}^{\dagger} / K}^{\dagger} \otimes_{\mathcal{D}_{\mathcal{X}^{\dagger} / K}} \mathcal{O}_{\mathcal{X}^{\dagger} / K} \rightarrow \mathcal{O}_{\mathcal{X}^{\dagger} / K}
$$

est un isomorphisme.

Démonstration. La question est locale, soit $\left(x_{1}, \ldots, x_{n}\right)$ est un système de coordonnées locales au-dessus d'un ouvert $U$ ayant la propriété (diff) d'algèbre $A^{\dagger}$ et $\partial_{1}, \ldots, \partial_{n}$ la base des champs de vecteurs tangents associés. Il suffit de montrer que les idéaux d'augmentation :

$$
\mathcal{D}_{\mathcal{X}^{\dagger} / K} \rightarrow \mathcal{O}_{\mathcal{X}^{\dagger} / K}, \mathcal{D}_{\mathcal{X}^{\dagger} / K}^{\dagger} \rightarrow \mathcal{O}_{\mathcal{X}^{\dagger} / K}
$$

sont engendrés par $\partial_{1}, \ldots, \partial_{n}$. C'est élémentaire dans le cas des opérateurs d'ordre fini mais beaucoup plus délicat dans le cas des opérateurs d'ordre infini. Soit $P$ un opérateur d'ordre infini tel que $P(1)=0$. En vertu de la première partie du théorème du symbole total ([13, Thm. 5.1]) l'opérateur $P$ est une somme infini

$$
\sum_{1 \leq|\beta| \leq \infty} a_{\beta}(x) \Delta^{\beta}
$$

où $a_{\beta}$ est une suite d'éléments de $A^{\dagger}$ telle que si

$$
\left(V\left[Y_{1}, \ldots, Y_{m}\right]\right)^{\dagger} \rightarrow A^{\dagger} \rightarrow 0
$$

est une présentation, il existe un nombre $\rho>1$ tel que $a_{\beta} \in A_{\rho}^{\dagger}$, un nombre $\lambda<1$ et une constante $C>0$ tels que l'ont ait les majorations

$$
\left\|a_{\beta}\right\|_{\rho} \leq C \lambda^{|\beta|} \text {. }
$$

On peut effectuer formellement la division de l'opérateur $P$ par l'idéal $\partial_{1}, \ldots, \partial_{n}$

$$
P=Q_{1} \partial_{1}+\cdots+Q_{n} \partial_{n}
$$

où

$$
Q_{1}=\sum_{\beta, \beta_{1} \neq 0} a_{\beta} / \beta_{1} \Delta^{\beta-(1, \ldots, 0)}
$$


Mais on peut trouver un nombre $\lambda^{\prime}, \lambda^{\prime}<1$ et une constante $C^{\prime}>0$ tels que l'on ait les majorations

$$
\left\|a_{\beta} / \beta_{1}\right\|_{\rho} \leq C^{\prime} \lambda^{\prime|\beta|-1}
$$

En vertu de la seconde partie du théorème de l'opérateur différentiel d'un symbole total ([13, Thm. 6.1]) la série $Q_{1}$ est un opérateur d'ordre infini et il en est de même des opérateurs $Q_{2}, \ldots, Q_{n}$ par le même raisonnement. Ceci montre que l'hypercohomologie du complexe

$$
\mathbf{R} \mathcal{H}_{o m_{\mathcal{D}^{\dagger} / K}}\left(\mathcal{O}_{\mathcal{X}^{\dagger} / K}, \mathcal{O}_{\mathcal{X}^{\dagger} / K}\right)
$$

est canoniquement isomorphe à l'hypercohomologie du complexe

$$
\mathbf{R} \mathcal{H o m}_{\mathcal{D}_{\mathcal{X}^{\dagger} / K}^{\dagger}}\left(\mathcal{O}_{\mathcal{X}^{\dagger} / K}, \mathcal{O}_{\mathcal{X}^{\dagger} / K}\right)
$$

qui implique le théorème de comparaison 7.2.1.

Corollaire 7.2.4. Pour tout $\mathcal{D}_{\mathcal{X}^{\dagger} / K^{-}}^{\dagger}$ module à gauche $\mathcal{M}^{\dagger}$ on a les isomorphismes canoniques :

$$
\operatorname{Ext}_{\mathcal{D}_{\mathcal{X}^{\dagger} / K}^{\bullet}}\left(\mathcal{O}_{\mathcal{X}^{\dagger} / K}, \mathcal{M}^{\dagger}\right) \simeq \operatorname{Ext}_{\mathcal{D}_{\mathcal{X}^{\dagger} / K}^{\dagger}}^{\bullet}\left(\mathcal{O}_{\mathcal{X}^{\dagger} / K}, \mathcal{M}^{\dagger}\right)
$$

En particulier on a les isomorphismes canoniques :

$$
\mathrm{H}_{\mathrm{dR}}^{\bullet}(\mathrm{X} / \mathrm{K}) \simeq \operatorname{Ext}_{\mathcal{D}_{\mathcal{X}^{\dagger} / \mathrm{K}}}^{\bullet}\left(\mathcal{O}_{\mathcal{X}^{\dagger} / \mathrm{K}}, \mathcal{O}_{\mathcal{X}^{\dagger} / \mathrm{K}}\right) \simeq \operatorname{Ext}_{\mathcal{D}_{\mathcal{X}^{\dagger} / \mathrm{K}}^{\dagger}}^{\bullet}\left(\mathcal{O}_{\mathcal{X}^{\dagger} / \mathrm{K}}, \mathcal{O}_{\mathcal{X}^{\dagger} / \mathrm{K}}\right)
$$

La démonstration précédente montre :

Proposition 7.2.5. Le complexe de Spencer étendu

$$
\mathcal{D}_{\mathcal{X}^{\dagger} / K}^{\dagger} \otimes_{\mathcal{D}_{\mathcal{X}^{\dagger} / K}} \operatorname{Spen}^{\bullet}\left(\mathcal{O}_{\mathcal{X}^{\dagger} / K}\right)
$$

est une résolution canonique du fibré trivial par des $\mathcal{D}_{\mathcal{X}^{\dagger} / K^{-}}^{\dagger}$ modules à gauche localement libres de type fini.

Démonstration. C'est une conséquence de la platitude de l'extension $\mathcal{D}_{\mathcal{X}^{\dagger} / K}$ $\rightarrow \mathcal{D}_{\mathcal{X}^{\dagger} / K}^{\dagger}$ et de l'isomorphisme précédent :

$$
\mathcal{D}_{\mathcal{X}^{\dagger} / K}^{\dagger} \otimes_{\mathcal{D}_{\mathcal{X}^{\dagger} / K}} \mathcal{O}_{\mathcal{X}^{\dagger} / K} \simeq \mathcal{O}_{\mathcal{X}^{\dagger} / K}
$$

Monsky-Washnitzer ont montré [16] pour une variété affine non singulière $X$ sur $k$ que les $K$-espaces $\mathrm{H}_{\mathrm{dR}}^{\bullet}(\mathrm{X} / \mathrm{K})$ ne dépendent à isomorphisme canonique près que de $X$ et fournissent un foncteur contravariant 
de la catégorie Affsm/k des variétés affines lisses sur le corps résiduel dans la catégorie homotopique $\mathrm{K}(K)$ des $K$-espaces vectoriels, parce que toute variété affine lisse sur $k$ se relève en un schéma †-adique affine lisse sur $V$.

La définition de la cohomologie de de Rham $p$-adique d'une variété algébrique $X$ (schéma de type fini et séparé sur $k[20])$ lisse sur $k([22, \S 17])$ admettant un relèvement †-adique global $\mathcal{X}^{\dagger}:=\left(X, \mathcal{O}_{\mathcal{X}^{\dagger} / V}\right)$ plat sur $V$ comme les espaces

$$
\operatorname{Ext}_{\mathcal{D}_{\mathcal{X}^{\dagger} / K}^{\dagger}}^{\bullet}\left(\mathcal{O}_{\mathcal{X}^{\dagger} / K}, \mathcal{O}_{\mathcal{X}^{\dagger} / K}\right)
$$

est proposée dans l'article de recherche ([11, page 303]). Mais il n'était pas montré l'indépendance de cette cohomologie du relèvement pour une variété lisse non affine et que cela définit un foncteur contravariant de la catégorie de toutes les variétés lisses sur $k$ qui se relèvent vers la catégorie dérivée $\mathrm{D}(K)$ des $K$-espaces vectoriels. Cependant on a le théorème fondamental qui définit les nombres de Betti $p$-adiques de $X$ :

Théorème 7.2.6. Sous la condition de relèvement global plat, les K-espaces vectoriels $\mathrm{Ext}_{\mathcal{D}_{\mathcal{X}^{\dagger} / K}^{\dagger}}^{\bullet}\left(\mathcal{O}_{\mathcal{X}^{\dagger} / K}, \mathcal{O}_{\mathcal{X}^{\dagger} / K}\right)$ sont de dimension finie.

Démonstration. En effet la suite spectrale locale-globale d'un recouvrement fini par des ouverts affines, ou même simplement en raisonnant par récurrence sur le nombre d'ouvert du recouvrements à l'aide de la suite de Mayer-Vietoris, ramène la finitude de ces espaces au cas affine [10] qui est le résultat central de la théorie.

Ce résultat est une indication très crédible qu'on était dès les années 1985, où l'on commencé à travailler dans ce domaine [11], sur la bonne voie et notre expérience des 25 dernières années a confirmé ce fait.

\section{Références}

[1] Arabia, A. : Relèvements des algèbres lisses et de leurs morphismes. Comment. Math. Helv. 76 (2001), 607-639.

[2] Berthelot, P. : Cohomologie rigide et théorie des $\mathcal{D}$-modules. In $p$-adic analysis (Trento, 1989), 80-124. Lecture Notes in Math. 1454. Springer, Berlin, 1990.

[3] Bourbaki, N. : Éléments de mathématique. Algèbre commutative, chapitres III-IV. Actualités Scientifiques et Industrielles 1293. Hermann, Paris 1961.

[4] Briançon, J. and Maisonobe, Ph. : Idéaux de germes d'opérateurs différentiels à une variable. Enseign. Math. (2) 30 (1984), 7-38.

[5] Chase, A. : On the homological dimension of algebras of differential operators. Comm. Algebra 1 (1974), 351-363. 
[6] Castro, P. : Calcul de la dimension et des multiplicités d'un $D$-module monogène. C. R. Acad. Sci. Paris Sér. I Math. 302 (1986), 487-490.

[7] Elkik, R. : Solutions d'équations à coefficients dans un anneau hensélien. Ann. Sci. École Norm. Sup. (4) 6 (1973), 553-603 (1974).

[8] Hauser, H. ANd Müller, M. : A rank theorem for analytic maps between power series spaces. Inst. Hautes Études Sci. Publ. Math.80 (1994), 96-115 (1995).

[9] Hauser, H. and Narváez-Macarro, L. : Continuous division of differential operators. Ann. Inst. Fourier (Grenoble) 51 (2001), no. 3, 769-778.

[10] Mebkhout, Z. : Sur le théorème de finitude de la cohomologie $p$-adique d'une variété affine non singulière. Amer. J. Math. 119 (1997), 1027-1081.

[11] Mebrhout, Z. and Narváez-Macarro, L. : Sur les coefficients de de Rham-Grothendieck des variétés algébriques. In p-adic Analysis (Trento, 1989), 267-309. Lecture Notes in Math. 1454. Springer, Berlin, 1990.

[12] Mebkhout, Z. and Narváez-Macarro, L. : La théorie du polynôme de Bernstein-Sato pour les algèbres de Tate et de Dwork-Monsky-Washnitzer. Ann. Sci. École Norm. Sup. (4) 24 (1991), 227-256.

[13] Mebkhout, Z. and Narváez-Macarro, L. : Le théorème du symbole total pour un opérateur différentiel p-adique. Rev. Mat. Iberoam. 26 (2010), no. $3,825-859$.

[14] Mebkhout, Z. and Narváez-Macarro, L. : Le théorème de continuité de la division dans les anneaux d'opérateurs différentiels. J. Reine Angew. Math. 503 (1998), 193-236.

[15] Meredith, D. : Weak formal schemes. Nagoya Math. J. 45 (1972), 1-38.

[16] Monsky, P. and Washnitzer, G. : Formal cohomology. I. Ann. of Math. (2) 88 (1968), 181-217

[17] Narváez Macarro, L. : Division theorem over the Dwork-MonskyWashnitzer completion of polynomial rings and Weyl algebras. In Rings, Hopf algebras, and Brauer groups (Antwerp/Brussels, 1996), 175-191. Lecture Notes in Pure and Appl. Math. 197. Dekker, New York, 1998.

[18] Noot-Huyghe, C. : Un théorème de comparaison entre les faisceaux d'opérateurs différentiels de Berthelot et Mebkhout-Narváez-Macarro. J. Algebraic Geom. 12 (2003), no. 1, 147-199.

[19] Smith, S. P. : The global homological dimension of ring of differential operators on a nonsingular variety over of field of positive characteristic. J. Algebra 107 (1987), 98-105.

[20] Grothendieck, A. And Dieudonné, J. : Éléments de Géométrie Algébrique. I. Inst. Hautes Études Sci. Publ. Math., no. 4, 1960.

[21] Grothendieck, A. And Dieudonné, J. : Éléments de Géométrie Algébrique. III, première partie. Inst. Hautes Études Sci. Publ. Math., no. 11, 1961. 
[22] Grothendieck, A. And Dieudonné, J. : Éléments de Géométrie Algébrique. IV, quatrième partie. Inst. Hautes Études Sci. Publ. Math., no. 32, 1967.

Recibido: 6 de noviembre de 2008

Revisado: 30 de marzo de 2010

Zoghman Mebkhout

UFR de Mathématiques

Université de Paris 7

175 rue de Chevaleret, F-75013, Paris

mebkhout@math.jussieu.fr 